\title{
Concentration of ground states in stationary Mean-Field Games systems
}

\author{
Annalisa Cesaroni and Marco Cirant
}

\begin{abstract}
In this paper we provide the existence of classical solutions to stationary mean field game systems in the whole space $\mathbb{R}^{N}$, with coercive potential and aggregating local coupling, under general conditions on the Hamiltonian. The only structural assumption we make is on the growth at infinity of the coupling term in terms of the growth of the Hamiltonian. This result is obtained using a variational approach based on the analysis of the non-convex energy associated to the system. Finally, we show that in the vanishing viscosity limit mass concentrates around the flattest minima of the potential. We also describe the asymptotic shape of the rescaled solutions in the vanishing viscosity limit, in particular proving the existence of ground states, i.e. classical solutions to mean field game systems in the whole space without potential, and with aggregating coupling.
\end{abstract}

AMS-Subject Classification. 35J50, 49N70, 35J47, 91A13, 35B25

Keywords. Ergodic Mean-Field Games, Semiclassical limit, Concentration-compactness method, Mass concentration, Elliptic systems, Variational methods.

\section{Contents}

1 Introduction 2

2 Some preliminary regularity results 6

2.1 The Hamilton-Jacobi-Bellman equation on the whole space . . . . . . . . . . . 8

2.2 A priori estimates for the Kolmogorov equation . . . . . . . . . . . . . . 11

3 Regularization procedure and existence of approximate solutions for $\varepsilon>0 \quad 13$

3.1 The regularized problem . . . . . . . . . . . . . . . . 13

3.2 A priori estimates and energy bounds . . . . . . . . . . . . 14

3.3 Existence of a solution . . . . . . . . . . . . . . . . . . . . 15

4 Existence of a solution to the MFG system for $\varepsilon>0$

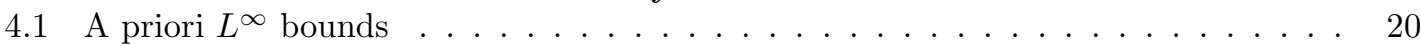

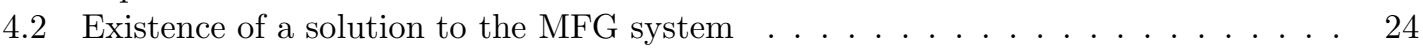

5 Concentration phenomena 2

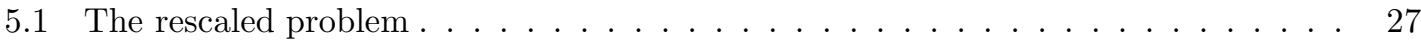

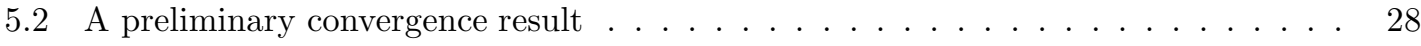

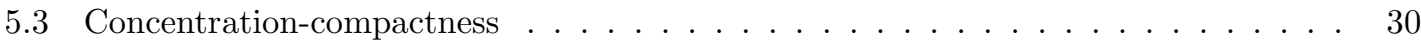

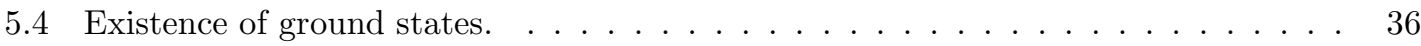

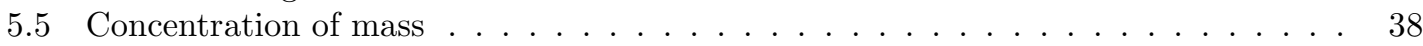

References 


\section{Introduction}

We consider a class of ergodic Mean-Field Games systems set on the whole space $\mathbb{R}^{N}$ with unbounded decreasing coupling: our problem is, given $\varepsilon>0$ and $M>0$, to find a constant $\lambda \in \mathbb{R}$ for which there exists a couple $(u, m) \in C^{2}\left(\mathbb{R}^{N}\right) \times W^{1, p}\left(\mathbb{R}^{N}\right)$, for any $p>1$, solving

$$
\left\{\begin{array}{l}
-\varepsilon \Delta u+H(\nabla u)+\lambda=f(m)+V(x) \\
-\varepsilon \Delta m-\operatorname{div}(m \nabla H(\nabla u))=0 \\
\int_{\mathbb{R}^{N}} m=M .
\end{array} \quad \text { on } \mathbb{R}^{N},\right.
$$

The aim of this work is two-fold. Firstly, for any fixed $\varepsilon>0$, we prove the existence of classical ground states of (1.1). Secondly, we study their behavior in the vanishing viscosity limit $\varepsilon \rightarrow 0$.

The Hamiltonian $H: \mathbb{R}^{N} \rightarrow \mathbb{R}$ is strictly convex, $H \in C^{2}\left(\mathbb{R}^{N} \backslash\{0\}\right)$ and has superlinear growth: we assume that there exist $C_{H}>0, K>0$ and $\gamma>1$ such that, for all $p \in \mathbb{R}^{N}$,

$$
\begin{aligned}
& C_{H}|p|^{\gamma}-K \leq H(p) \leq C_{H}|p|^{\gamma}, \\
& \nabla H(p) \cdot p-H(p) \geq K^{-1}|p|^{\gamma}-K \quad \text { and } \quad|\nabla H(p)| \leq K|p|^{\gamma-1} .
\end{aligned}
$$

The coupling term $f:[0,+\infty) \rightarrow \mathbb{R}$ is a locally Lipschitz continuous function such that there exist $C_{f}>0$ and $K>0$ for which

$$
-C_{f} m^{\alpha}-K \leq f(m) \leq-C_{f} m^{\alpha}+K
$$

with

$$
0<\alpha<\frac{\gamma}{N(\gamma-1)}=\frac{\gamma^{\prime}}{N}
$$

where $\gamma^{\prime}=\frac{\gamma}{\gamma-1}$ is the conjugate exponent of $\gamma$.

Finally, we assume that the potential $V$ is a locally Hölder continuous function, and that there exist $b>0$ and a constant $C_{V}>0$ such that

$$
C_{V}^{-1}\left(\max \left\{|x|-C_{V}, 0\right\}\right)^{b} \leq V(x) \leq C_{V}(1+|x|)^{b} .
$$

Note that the requirement of $V$ to be non-negative is not crucial, we just need it to be bounded from below.

Mean-Field Games (MFG) is a recent theory that models the behaviour of a very large number of indistinguishable rational agents aiming at minimizing a common cost. The theory was introduced in the seminal works by Lasry, Lions [22, 23, 24, 25] and by Huang, Caines, Malhamé [18, and has been rapidly growing during the last decade due to its mathematical challenges and several potential applications (from economics and finance, to engineering and models of social systems). In the ergodic MFG setting, the dynamics of a typical agent is given by the controlled stochastic differential equation

$$
d X_{s}=-v_{s} d s+\sqrt{2 \varepsilon} d B_{s}, s>0,
$$

where $v_{s}$ is the control and $B_{s}$ is a Brownian motion, with initial state given by a random variable $X_{0}$. The cost (of long-time average form) is given by

$$
\lim _{T \rightarrow \infty} \frac{1}{T} \mathbb{E} \int_{0}^{T}\left[L\left(v_{s}\right)+V\left(X_{s}\right)+f\left(m\left(X_{s}\right)\right)\right] d s,
$$

where the Lagrangian $L$ is the Legendre transform of $H$ (see (2.1)) and $m(x)$ denotes the density of population of small agents at a position $x \in \mathbb{R}^{N}$. A typical agent minimizes his own cost, and the density of its corresponding distribution law $\mathcal{L}\left(X_{s}\right)$ converges as time $s \rightarrow \infty$ to a stationary density $\mu$, which is independent of the initial distribution $\mathcal{L}\left(X_{0}\right)$. In an equilibrium regime, $\mu$ coincides with the population density $m$. This equilibrium is encoded from the PDE viewpoint 
in (1.1): a solution $u$ of the Hamilton-Jacobi-Bellman equation gives an optimal control for the typical agent in feedback form $\nabla H(\nabla u(\cdot))$, and the Kolmogorov equation provides the density $m$ of the agents playing in an optimal way.

The two key points of our setting are the following: firstly, the cost is monotonically decreasing with respect to the population distribution $m$, namely agents are attracted toward congested areas. A large part of the MFG literature focuses on the study of systems with competition, namely when the coupling in the cost is monotonically increasing; this assumption is essential if one seeks for uniqueness of equilibria, and it is in general crucial in many existence and regularity arguments, see, e.g [17, and references therein. On the other hand, models with aggregation like (1.1) have been considered in few cases, see [10, 12, 13, 14, 15.

Secondly, the state of a typical agent here is the whole euclidean space $\mathbb{R}^{N}$. Usually, the analysis of (1.1) is carried out in the periodic setting, in order to avoid boundary issues and the non-compactness of $\mathbb{R}^{N}$. Few investigations are available in the truly non-periodic setting: see [29] for time-dependent problems, 22 for the case of bounded controls, [16] for some regularity results and 3] for the Linear-Quadratic framework. We observe that the non-compact setting is even more delicate for stationary (ergodic) problems like (1.1): a stable long-time regime of a typical player is ensured if the Brownian motion is compensated by the optimal velocity $v_{s}$. In other words, if a force that drives players to bounded states is missing, dissipation eventually leads their distribution to vanish on the whole $\mathbb{R}^{N}$. This phenomenon is impossible if the state space is compact. The main issue here is that the behaviour of the optimal velocity $v_{s}(\cdot)=\nabla H(\nabla u(\cdot))$ is a priori unknown, and depends in an implicit way on $V$ and the distribution $m$ itself. Note that $V(\cdot)$ represents the spatial preference of a single agent; if it grows as $|x| \rightarrow \infty$, it discourages agents to be far away from the origin. At the PDE level, this will compensate the lack of compactness of $\mathbb{R}^{N}$. Let us mention that even without the coupling term $f\left(m^{\alpha}\right)$, the ergodic control problem in unbounded domains has received a considerable attention, see e.g. 4, 19, 20, and references therein.

In our analysis, we exploit the variational nature of the system (1.1), which has been pointed out already in the first papers on MFG, see 24, or the more recent work 27. Indeed, solutions to (1.1) can be put in correspondence with critical points of the following energy

$$
\mathcal{E}(m, w):= \begin{cases}\int_{\mathbb{R}^{N}} m L\left(-\frac{w}{m}\right)+V(x) m+F(m) d x & \text { if }(m, w) \in \mathcal{K}_{\varepsilon, M}, \\ +\infty & \text { otherwise }\end{cases}
$$

where $F(m)=\int_{0}^{m} f(n) d n$ for $m \geq 0$ and $F(m)=0$ for $m \leq 0$ and

$$
L\left(-\frac{w}{m}\right):= \begin{cases}\sup _{p \in \mathbb{R}^{N}}\left(-\frac{p \cdot w}{m}-H(p)\right) & \text { if } m>0, \\ 0 & \text { if } m=0, w=0 \\ +\infty & \text { otherwise. }\end{cases}
$$

Note that $m L(-\cdot / m)$ reads as the Legendre transform of $m H(\cdot)$. The constraint set is defined as

$$
\begin{aligned}
& \mathcal{K}_{\varepsilon, M}:=\left\{(m, w) \in L^{1}\left(\mathbb{R}^{N}\right) \cap L^{q}\left(\mathbb{R}^{N}\right) \times L^{1}\left(\mathbb{R}^{N}\right)\right. \text { s.t. } \\
& \varepsilon \int_{\mathbb{R}^{N}} m(-\Delta \varphi) d x=\int_{\mathbb{R}^{N}} w \cdot \nabla \varphi d x \quad \forall \varphi \in C_{0}^{\infty}\left(\mathbb{R}^{N}\right), \\
& \left.\int_{\mathbb{R}^{N}} m d x=M, \quad m \geq 0 \text { a.e. }\right\} \quad \text { with } q= \begin{cases}\frac{N}{N-\gamma^{\prime}+1} & \gamma^{\prime} \leq N \\
\gamma^{\prime} & \gamma^{\prime}>N .\end{cases}
\end{aligned}
$$

Under assumption (1.3) on the coupling term, the energy $\mathcal{E}$ is not convex. Condition (1.4) is necessary for the problem $e_{\mathcal{E}}(M):=\min _{(m, w) \in \mathcal{K}_{\varepsilon, M}} \mathcal{E}(m, w)$ to be well-posed. Indeed, consider any $\left(m_{0}, w_{0}\right) \in \mathcal{K}_{\varepsilon, M}$ such that $m_{0}$ has compact support. An easy computation shows that if $\alpha>\gamma^{\prime} / N$, then

$$
\mathcal{E}\left(\sigma^{-N} m_{0}\left(\sigma^{-1} \cdot\right), \sigma^{-(N+1)} w_{0}\left(\sigma^{-1} \cdot\right)\right) \rightarrow-\infty
$$


as $\sigma \rightarrow 0$, so $\mathcal{E}$ is not bounded from below on $\mathcal{K}_{\varepsilon, M}$. We show that (1.4) is indeed sufficient for $e_{\varepsilon}(M)$ to be finite, and allows to look for ground states of (1.1). This will be accomplished by a study of the Sobolev regularity of the Kolmogorov equation, see in particular Section 2.2. Note that the critical case $\alpha=\gamma^{\prime} / N$ is more delicate, and requires additional analysis. We also mention that another critical exponent is intrinsic in (1.1): if $\alpha>\gamma^{\prime} /\left(N-\gamma^{\prime}\right)$, one has to expect nonexistence of solutions (see [12). We refer to our case as the subcritical case, in analogy with the $L^{2}$-subcritical regime in nonlinear Schrödinger equations with prescribed mass (see [12, Remark $2.9]$ for additional comments). The analogy can be made precise in the purely quadratic framework, that is when $H(p)=\frac{1}{2}|p|^{2}$. Indeed, as observed in [22, 23], the so-called Hopf-Cole transformation permits to reduce the number of unknowns in the system. Setting $v^{2}(x):=m(x)=c e^{-\frac{u(x)}{\varepsilon}}$, with $c$ normalizing constant, then $v$ is a solution to

$$
-2 \varepsilon^{2} \Delta v+(V(x)-\lambda) v=-f\left(v^{2}\right) v
$$

with $\int_{\mathbb{R}^{N}} v^{2}(x) d x=M$. Then the energy reads $\mathcal{E}(v)=\int_{\mathbb{R}^{N}} \varepsilon^{2}|\nabla v|^{2}+\frac{1}{2} V(x) v^{2}+\frac{1}{2} F\left(v^{2}\right) d x$.

In our approach, to construct solutions to (1.1), we look for minimizers $(m, w) \in \mathcal{K}_{\varepsilon, M}$ of the energy (1.6). These minimizers can be obtained by classical direct methods, by using in particular estimates and compactness in some $L^{p}$ space for elements $(m, w)$ in $\mathcal{K}_{\varepsilon, M}$ with bounded action, i.e. which satisfy $\int_{\mathbb{R}^{N}} m L\left(-\frac{w}{m}\right) d x \leq C$, obtained in Section 2.2 Then, the existence of a solution $\left(u_{\varepsilon}, \lambda_{\varepsilon}\right)$ of the HJB equation in (1.1) is obtained by considering another functional with linearized coupling (around the minimizer) and the associated dual functional in the sense of Fenchel-Rockafellar (as in [8]). One has to take care of the interplay between $u$ and $m$ as $|x| \rightarrow \infty$. To handle the lack of a priori regularity on the function $m$, we first regularize the problem, by applying standard regularizing convolution kernels on the coupling (see Section 3). We construct minimizers $\left(m_{k}, w_{k}\right)$ of the regularized energy and associated solutions $\left(u_{k}, m_{k}\right)$ of the regularized version of (1.1). Then, in order to come back to the initial problem, we provide some new a priori uniform $L^{\infty}$ bounds on $m_{k}$, which in turn imply a priori uniform bounds on $\left|\nabla u_{k}\right|$ and (local) Hölder regularity of $m_{k}$ that is uniform in $k$. This key a priori bound is provided by Theorem 4.1

Note that we will consider classical solutions to this system (with a slight abuse of terminology), that is $(u, m) \in C^{2}\left(\mathbb{R}^{N}\right) \times W^{1, p}\left(\mathbb{R}^{N}\right)$, for all $p>1$. The existence result, proved in Section 4, is the following.

Theorem 1.1. Under the assumptions (1.2), (1.3), (1.4) and (1.5), for every $\varepsilon>0$ there exists a classical solution $\left(u_{\varepsilon}, m_{\varepsilon}, \lambda_{\varepsilon}\right) \in C^{2}\left(\mathbb{R}^{N}\right) \times W^{1, p}\left(\mathbb{R}^{N}\right) \times \mathbb{R}$, for all $p>1$, to (1.1). Moreover, $\left(m_{\varepsilon},-m_{\varepsilon} \nabla H\left(\nabla u_{\varepsilon}\right)\right)$ is a minimizer in the set $\mathcal{K}_{\varepsilon, M}$ of the energy (1.6).

We observe (see Remarks 3.5 4.2) that Theorem 1.1 holds under more general conditions on $H$ and $f$, that is, if there exist $C_{H}, C_{f}>0$ and $K>0$ such that

$$
C_{H}^{-1}|p|^{\gamma}-K \leq H(p) \leq C_{H}\left(|p|^{\gamma}+1\right), \quad-C_{f} m^{\alpha}-K \leq f(m) \leq C_{f}^{-1} m^{\alpha}+K,
$$

where $\alpha$ satisfies (1.4).

In the second part of the work, in Section 5, we analyze the behavior of the triple $\left(u_{\varepsilon}, \lambda_{\varepsilon}, m_{\varepsilon}\right)$ coming from a minimizer of $\mathcal{E}$ as $\varepsilon \rightarrow 0$, under the assumptions (1.2), (1.3). From the viewpoint of the model, this amounts to remove the Brownian noise from the agents' dynamics. Heuristically, if the diffusion becomes negligible, one should observe aggregation of players (induced by the decreasing monotonicity of coupling in the cost) towards minima of the potential $V$, that are the preferred sites. Moreover, in the case $V$ has a finite number of minima and polynomial behavior (that is, when (1.13) holds) we specialize the result showing that the limit procedure selects the more stable minima of $V$, implying e.g. full convergence in the case that there exists a unique flattest minimum.

In order to bring as much as possible information to the limit, we consider an appropriate rescaling of $m, u$, namely

$$
\bar{m}_{\varepsilon}(\cdot)=\varepsilon^{\frac{N \gamma^{\prime}}{\gamma^{\prime}-\alpha N}} m\left(\varepsilon^{\frac{\gamma^{\prime}}{\gamma^{\prime}-\alpha N}} \cdot+x_{\varepsilon}\right), \quad \bar{u}_{\varepsilon}(\cdot)=\varepsilon^{\frac{N \alpha\left(\gamma^{\prime}-1\right)-\gamma^{\prime}}{\gamma^{\prime}-\alpha N}}\left(u\left(\varepsilon^{\frac{\gamma^{\prime}}{\gamma^{\prime}-\alpha N}} \cdot+x_{\varepsilon}\right)-u\left(x_{\varepsilon}\right)\right),
$$


for all $\varepsilon>0$. The rescaling is designed so that $\left(\bar{u}_{\varepsilon}, \bar{m}_{\varepsilon}\right)$ solves a MFG system where the nonlinearities have the same behavior of the original ones, i.e. $H_{\varepsilon} \sim|p|^{\gamma}$ as $p \rightarrow \infty$, but the coefficient in front of the Laplacian is equal to one for all $\varepsilon$, see (5.19). Moreover, the couple $\bar{u}_{\varepsilon}, \bar{m}_{\varepsilon}$ is associated to a minimizer of a rescaled energy $\mathcal{E}_{\varepsilon}$, see (5.23). It turns out that in this rescaling process, the potential $V$ becomes

$$
V_{\varepsilon}(\cdot)=\varepsilon^{\frac{N \alpha \gamma^{\prime}}{\gamma^{\prime}-\alpha N}} V\left(\varepsilon^{\frac{\gamma^{\prime}}{\gamma^{\prime}-\alpha N}} \cdot\right)
$$

and vanishes (locally) as $\varepsilon \rightarrow 0$. Therefore, as one passes to the limit, the potential cannot compensate anymore the lack of compactness of $\mathbb{R}^{N}$, and the convergence of $\bar{m}_{\varepsilon}$ in $L^{1}\left(\mathbb{R}^{N}\right)$ has to be proven by other methods. Heuristically, the aggregating force should be strong enough to overcome the dissipation effect, but the clustering point can be hard to predict by lack of spatial preference. This is why we also have to translate in (1.10) by $x_{\varepsilon}$. We will select $x_{\varepsilon}$ to be the minimum of $u_{\varepsilon}$ : heuristically, being $u_{\varepsilon}$ the value function, this is the point where most of the players should be located. In order to recover compactness for the sequence $\bar{m}_{\varepsilon}$, we implement some ideas of the celebrated concentration-compactness method [26]. This principle states intuitively that if loss of compactness occurs, $\bar{m}_{\varepsilon}$ splits in (at least) two parts which are going infinitely far away from each other, that is

$$
\bar{m}_{\varepsilon} \sim \chi_{B_{R}(0)} \bar{m}_{\varepsilon}+\chi_{\mathbb{R}^{N} \backslash B_{2 R}(0)} \bar{m}_{\varepsilon}
$$

with $R \rightarrow \infty, \int \chi_{B_{R}(0)} \bar{m}_{\varepsilon} \sim a$ and $\int \chi_{\mathbb{R}^{N} \backslash B_{2 R}(0)} \bar{m}_{\varepsilon} \sim M-a$ for some $a \in(0, M)$ (a third possibility might happen, but it is easily ruled out here by local estimates). This induces a splitting in the energy $\mathcal{E}$, that is

$$
\inf _{\int m=M} \mathcal{E}_{\varepsilon} \gtrsim \inf _{\int m=a} \mathcal{E}_{\varepsilon}+\inf _{\int m=M-a} \mathcal{E}_{\varepsilon} .
$$

One then exploits a special feature of $\mathcal{E}_{\varepsilon}$, which is called sub-additivity:

$$
\inf _{\int m=M} \mathcal{E}_{\varepsilon}<\inf _{\int m=a} \mathcal{E}_{\varepsilon}+\inf _{\int m=M-a} \mathcal{E}_{\varepsilon}
$$

that makes (1.12) impossible. While sub-additivity is easy to prove for $\mathcal{E}_{\varepsilon}$ (see Lemma 5.5), the splitting (1.12) requires technical work, in particular due to the presence of the term $m L(-w / m)$ in $\mathcal{E}_{\varepsilon}$, that becomes increasingly singular as $m$ approaches zero (a simple cut-off as in (1.11) is not useful). The property (1.12) is proven in Theorem 5.6. It relies on the Brezis-Lieb lemma and a perturbation argument. The $L^{1}$ convergence of $\bar{m}_{\varepsilon}$ enables us to obtain the full convergence of $\left(\bar{u}_{\varepsilon}, \bar{m}_{\varepsilon}\right)$ to a limit MFG system. By a uniform control of the decay of $\bar{m}_{\varepsilon}$ as $|x| \rightarrow \infty$, that comes from a Lyapunov function built upon $\bar{u}_{\varepsilon}$, energy arguments and the crucial $L^{\infty}$ estimate of Theorem 4.1 we are also able to keep track of $x_{\varepsilon}$. In terms of the non-rescaled density $m_{\varepsilon}, x_{\varepsilon}$ is the point around which most of the mass is located.

The second main result of this work is stated in the following two theorems. The first one is about concentration of $m_{\varepsilon}$.

Theorem 1.2. Under the assumptions of Theorem 1.1, there exist sequences $\varepsilon \rightarrow 0$ and $x_{\varepsilon}$, such that for all $\eta>0$ there exists $R$ and $\varepsilon_{0}$ for which for all $\varepsilon<\varepsilon_{0}$,

$$
\int_{\left|x-x_{\varepsilon}\right| \leq R \varepsilon \frac{\gamma^{\prime}}{\gamma^{\prime}-\alpha N}} m_{\varepsilon} d x \geq M-\eta
$$

Moreover, $x_{\varepsilon} \rightarrow \bar{x}$, where $V(\bar{x})=0$, i.e. $\bar{x}$ is a minimum of $V$.

If, in addition, $V$ has the form

$$
V(x)=h(x) \prod_{j=1}^{n}\left|x-x_{j}\right|^{b_{j}}, \quad C_{V}^{-1} \leq h(x) \leq C_{V} \text { on } \mathbb{R}^{N},
$$

for some $x_{j} \in \mathbb{R}^{N}$, and $b_{j}>0$ (with $\sum_{j=1}^{n} b_{j}=b$ ), then $x_{\varepsilon} \rightarrow x_{i}$, with $i \in\left\{j=1, \ldots, n \mid b_{j}=\right.$ $\left.\max _{k} b_{k}\right\}$. 
Secondly, we describe the asymptotic profile of $\left(\bar{u}_{\varepsilon}, \bar{m}_{\varepsilon}\right)$ as $\varepsilon \rightarrow 0$. Note that as a byproduct we obtain the existence of solutions to MFG systems without potential.

Theorem 1.3. Up to subsequences, $\left(\bar{u}_{\varepsilon}, \bar{m}_{\varepsilon}\right)$ converges in $C_{\text {loc }}^{1}\left(\mathbb{R}^{N}\right) \times C_{\text {loc }}\left(\mathbb{R}^{N}\right) \cap L^{p}\left(\mathbb{R}^{N}\right)$, for all $p \geq 1$, to a solution $(\bar{u}, \bar{m})$ of

$$
\left\{\begin{array}{l}
-\Delta u+C_{H}|\nabla u|^{\gamma}+\lambda=-C_{f} m^{\alpha} \\
-\Delta m-C_{H} \gamma \operatorname{div}\left(m|\nabla u|^{\gamma-2} \nabla u\right)=0 \\
\int_{\mathbb{R}^{N}} m=M .
\end{array}\right.
$$

The function $\bar{u}$ is globally Lipschitz continuous on $\mathbb{R}^{N}$, and there exists $c_{1}, c_{2}>0$ such that $0<\bar{m}(x) \leq c_{1} e^{-c_{2}|x|}$.

Finally, if $\bar{w}=-C_{H} \gamma \bar{m}|\nabla \bar{u}|^{\gamma-2} \nabla \bar{u}$, then

$$
\mathcal{E}_{0}(\bar{m}, \bar{w})=\min \left\{\mathcal{E}_{0}(m, w) \mid(m, w) \in \mathcal{K}_{1, M}, m\left(1+|y|^{b}\right) \in L^{1}\left(\mathbb{R}^{N}\right)\right\},
$$

where

$$
\mathcal{E}_{0}(m, w)=\int_{\mathbb{R}^{N}} C_{L} \frac{|w|^{\gamma^{\prime}}}{m^{\gamma^{\prime}-1}}-\frac{1}{\alpha+1} m^{\alpha+1} d y
$$

We finally observe that by analogous methods, one can prove existence of solutions to more general potential-free MFG systems, see Remark 5.9.

\section{Notation}

We will intend a classical solution to the system (1.1) to be a triple $(u, m, \lambda) \in C^{2}\left(\mathbb{R}^{N}\right) \times$ $W^{1, p}\left(\mathbb{R}^{N}\right) \times \mathbb{R}$, for all $p>1$.

For any given $p>1$, we will denote by $p^{\prime}=\frac{p}{p-1}$ the conjugate exponent of $p, p^{*}=\frac{N p}{N-p}$ if $p<N$ and $p^{*}=+\infty$ if $p \geq N$.

For all $R>0, x \in \mathbb{R}^{N}, B_{R}(x):=\left\{y \in \mathbb{R}^{N}:|x-y|<R\right\}$. We will denote by $\omega_{N}:=\left|B_{1}(0)\right|$. Finally, $C, C_{1}, K, K_{1}, \ldots$ denote (positive) constants we need not to specify.

Acknowledgements. The authors are partially supported by the Fondazione CaRiPaRo Project "Nonlinear Partial Differential Equations: Asymptotic Problems and Mean-Field Games" and PRAT CPDA157835 of University of Padova "Mean-Field Games and Nonlinear PDEs".

\section{Some preliminary regularity results}

Let $L$ be the Legendre transform of $H$, i.e.

$$
L(q)=H^{*}(q)=\sup _{p \in \mathbb{R}^{N}}[p \cdot q-H(p)], \quad q \in \mathbb{R}^{N} .
$$

The assumptions on $H$ guarantee the following (see, e.g., [11, Proposition 2.1]).

Proposition 2.1. There exist $C_{L}, C_{1}, C_{2}>0$ depending on $C_{H}$ and on $\gamma$ such that $\forall p, q \in \mathbb{R}^{N}$,

i) $L \in C^{2}\left(\mathbb{R}^{N} \backslash\{0\}\right)$ and it is strictly convex,

ii) $0 \leq C_{L}|q|^{\gamma^{\prime}} \leq L(q) \leq C_{L}\left(|q|^{\gamma^{\prime}}+1\right)$,

iii) $\nabla L(q) \cdot q-L(q) \geq C_{1}|q|^{\gamma^{\prime}}-C_{1}^{-1}$,

iv) $\left.C_{1} q\right|^{\gamma^{\prime}-1}-C_{1}^{-1} \leq|\nabla L(q)| \leq C_{1}^{-1}\left(|q|^{\gamma^{\prime}-1}+1\right)$.

v) $C_{2}|p|^{\gamma-1}-C_{2}^{-1} \leq|\nabla H(p)| \leq C_{2}^{-1}\left(|p|^{\gamma-1}+1\right)$.

We will use the following (standard) result on Hölder functions vanishing at infinity. 
Lemma 2.2. Suppose that $m \geq 0,\|m\|_{C^{0, \theta}\left(\mathbb{R}^{N}\right)} \leq c_{h}$, for some $\theta, c_{h}>0$, and $\int_{\mathbb{R}^{N}} m d x<\infty$. Then, $m(x) \rightarrow 0$ as $|x| \rightarrow \infty$. Moreover, if

$$
\int_{|x| \geq R} m d x<\eta
$$

for some $\eta, R>0$, then

$$
\max _{|x| \geq R} m(x) \leq C \eta^{\frac{\theta}{\theta+N}}
$$

where $C>0$ depends only on $c_{h}, N$.

Proof. By contradiction, suppose that there exists $\delta>0$ and a sequence $\left|x_{n}\right| \rightarrow \infty$ such that $m\left(x_{n}\right)>\delta$ for all $n$. We may also assume that $\left|x_{n+1}\right| \geq\left|x_{n}\right|+1$ for all $n$. By the Hölder regularity assumption,

$$
m(x) \geq m\left(x_{n}\right)-c_{h}\left|x-x_{n}\right|^{\theta} \geq \frac{\delta}{2},
$$

provided that $x \in B_{r}\left(x_{n}\right)$, and $r^{\theta} \leq \frac{\delta}{2 c_{h}}$. Choose $r=\min \left\{1,\left(\frac{\delta}{2 c_{h}}\right)^{\frac{1}{\theta}}\right\}$, so that $B_{r}\left(x_{n}\right) \cap B_{r}\left(x_{m}\right)=\emptyset$ for all $n \neq m$. Then,

$$
\int_{\mathbb{R}^{N}} m d x \geq \sum_{n \in \mathbb{N}} \int_{B_{r}\left(x_{n}\right)} m d x \geq \sum_{n \in \mathbb{N}} \frac{\delta}{2}\left|B_{r}(0)\right|=+\infty
$$

that is impossible.

As for the second part, let $M:=\max _{|x| \geq R} m(x)=m(\bar{x}),|\bar{x}| \geq R$ (note that such a maximum is achieved as a consequence of the first part of the lemma). As before,

$$
m(x) \geq m(\bar{x})-c_{h}|x-\bar{x}|^{\theta} \geq \frac{M}{2}
$$

for all $x \in B_{r}(\bar{x})$, where $r=\left(\frac{M}{2 c_{h}}\right)^{1 / \theta}$. Therefore,

$$
\eta>\int_{|x| \geq R} m d x \geq \frac{M}{4}\left|B_{r}(\bar{x})\right|=\frac{M}{4}\left|B_{1}(0)\right|\left(\frac{M}{2 c_{h}}\right)^{N / \theta},
$$

and (2.2) follows.

We recall the following well known result, proved in [7, Theorem 1].

Theorem 2.3. Let $f_{n} \rightarrow f$ a.e. in $\mathbb{R}^{N}$ and assume that $\left\|f_{n}\right\|_{L^{p}\left(\mathbb{R}^{N}\right)} \leq C$ for all $n$ and for some $p \in[1,+\infty)$. Then

$$
\lim _{n}\left[\left\|f_{n}\right\|_{L^{p}\left(\mathbb{R}^{N}\right)}^{p}-\left\|f_{n}-f\right\|_{L^{p}\left(\mathbb{R}^{N}\right)}^{p}\right]=\|f\|_{L^{p}\left(\mathbb{R}^{N}\right)}^{p} .
$$

From classical elliptic regularity, we have the following result.

Proposition 2.4. Let $p>1$ and $m \in L^{p}\left(\mathbb{R}^{N}\right)$ be such that

$$
\left|\int_{\mathbb{R}^{N}} m \Delta \varphi d x\right| \leq K\|\nabla \varphi\|_{L^{p^{\prime}\left(\mathbb{R}^{N}\right)}} \quad \text { for all } \varphi \in C_{0}^{\infty}\left(\mathbb{R}^{N}\right)
$$

for some $K>0$. Then, $m \in W^{1, p}\left(\mathbb{R}^{N}\right)$ and there exists $C>0$ depending only on $p$, such that

$$
\|\nabla m\|_{L^{p}\left(\mathbb{R}^{N}\right)} \leq C K
$$


Proof. Fix any $R>1$. Let $\psi \in C_{0}^{\infty}\left(B_{2}(0)\right), \varphi(R x):=\psi(x)$ (so, $\left.\varphi \in C_{0}^{\infty}\left(B_{2 R}(0)\right)\right)$ and $v(x):=$ $m(R x)$ on $\mathbb{R}^{N}$. Then,

$$
\begin{array}{r}
\left|\int_{B_{2}(0)} v \Delta \psi d x\right|=R^{2-N}\left|\int_{B_{2 R}(0)} m \Delta \varphi d y\right| \leq K R^{2-N}\left(\int_{B_{2 R}(0)}|\nabla \varphi|^{p^{\prime}} d y\right)^{1 / p^{\prime}} \\
=K R^{1-N+N / p^{\prime}}\left(\int_{B_{2}(0)}|\nabla \psi|^{p^{\prime}} d x\right)^{1 / p^{\prime}} \leq K R^{1-N / p}\|\psi\|_{W^{1, p^{\prime}}\left(B_{2}(0)\right)}
\end{array}
$$

Hence, by [1, Theorem 6.1], $v \in W^{1, p}\left(B_{1}(0)\right)$ and there exists a constant $C$, depending on $p$ (but not on $R$ ), such that

$$
\|\nabla v\|_{L^{p}\left(B_{1}(0)\right)} \leq\|v\|_{W^{1, p}\left(B_{1}(0)\right)} \leq C\left(K R^{1-N / p}+\|v\|_{L^{p}\left(B_{2}(0)\right)}\right) .
$$

Therefore,

$$
\begin{array}{r}
\left(\int_{B_{R}(0)}|\nabla m|^{p} d y\right)^{1 / p}=R^{N / p-1}\left(\int_{B_{1}(0)}|\nabla v|^{p} d x\right)^{1 / p} \leq C\left[K+R^{N / p-1}\left(\int_{B_{2}(0)}|v|^{p} d x\right)^{1 / p}\right] \\
=C\left(K+R^{-1}\|m\|_{L^{p}\left(B_{2}(0)\right)}\right) .
\end{array}
$$

Letting $R \rightarrow \infty$, we get that $|\nabla m| \in L^{p}\left(\mathbb{R}^{n}\right)$ and the desired estimate.

\subsection{The Hamilton-Jacobi-Bellman equation on the whole space}

In this section we provide some a priori regularity estimates and existence results for HamiltonJacobi-Bellman equations in the whole spaces of ergodic type. In particular we will consider families of Hamilton-Jacobi-Bellman equations

$$
-\Delta u_{n}+H_{n}\left(\nabla u_{n}\right)+\lambda_{n}=F_{n}(x)-f_{n}(x) \quad \text { on } \mathbb{R}^{N}
$$

where $F_{n}-f_{n}$ is locally Hölder continuous, $\lambda_{n} \in \mathbb{R}$ are equibounded in $n$, that is $\left|\lambda_{n}\right| \leq \lambda$ and $f_{n} \in L^{\infty}\left(\mathbb{R}^{N}\right)$, with $\left\|f_{n}\right\|_{\infty} \leq c_{f}$ for some $c_{f}>0$ independent of $n$. Moreover $H_{n}$ is for every $n$ an Hamiltonian which satisfies (1.2), with constants $\gamma$ and $C_{H}$ independent of $n$; finally, there exists $C_{F} \geq 0$ and $b \geq 0$ independent of $n$ such that

$$
C_{F}^{-1}\left(\max \left\{|x|-C_{F}, 0\right\}\right)^{b} \leq F_{n}(x) \leq C_{F}(1+|x|)^{b} \quad \forall n \text { and } \forall x \in \mathbb{R}^{N} .
$$

Note that, differently from assumption (1.5) for the potential $V$, the function $F_{n}$ can also be bounded, if $b=0$.

Theorem 2.5. Let $u_{n} \in C^{2}\left(\mathbb{R}^{N}\right)$ be a sequence of classical solutions of the HJB equations (2.3). Then there exists a constant $K>0$ depending on $C_{H}, C_{F}, c_{f}, \gamma, N, \lambda$ such that

$$
\left|\nabla u_{n}(x)\right| \leq K(1+|x|)^{\frac{b}{\gamma}},
$$

where $b \geq 0$ is the growth of $F_{n}$ appearing in (2.4) and $\gamma$ is the growth of $H_{n}$ appearing in (1.2).

Proof. Without loss of generality we may consider $H_{n}(p)=C_{H}|p|^{\gamma}$ for all $n$ and $p$. Indeed, every $v_{n}$ solves

$$
-\Delta u_{n}+C_{H}\left|\nabla u_{n}\right|^{\gamma}+\lambda_{n}=F_{n}(x)-f_{n}(x)+C_{H}\left|\nabla u_{n}\right|^{\gamma}-H_{n}\left(\nabla u_{n}\right) \quad \text { on } \mathbb{R}^{N},
$$

and since $\left.\left|C_{H}\right| \nabla u_{n}\right|^{\gamma}-H_{n}\left(\nabla u_{n}\right) \mid \leq C_{H}$ by (1.2), we can redefine $f_{n}$ to include $C_{H}\left|\nabla u_{n}\right|^{\gamma}$ $H_{n}\left(\nabla u_{n}\right)$, which then satisfies the bound $\left\|f_{n}\right\|_{\infty} \leq c_{f}+C_{H}$. 
We first claim that if $v \in C^{2}\left(B_{2}(0)\right)$ satisfies

$$
\left.\left|-\Delta v+C_{H}\right| \nabla v\right|^{\gamma} \mid \leq k \quad \text { on } B_{2}(0)
$$

for some $k>0$, then we have for any $r \in[1, \infty]$,

$$
\|\nabla v\|_{L^{r}\left(B_{1}(0)\right)} \leq \widetilde{C},
$$

where $\widetilde{C}$ depends only on $k, C_{H}, \gamma, N, r$. If $r \in[1, \infty)$, this is proven in [21, Theorem A.1]. The case $r=\infty$ follows by classical elliptic regularity, since if $r$ in (2.6) is large enough, then $-\Delta v$ is bounded in $L^{q}\left(B_{3 / 2}(0)\right)$ for some $q>N$, and the statement follows by Sobolev embeddings.

In view of these considerations, the gradient bound (2.5) easily follows if $b=0$. For the case $b>0$, fix $x_{0} \in \mathbb{R}^{N}$, and let $\delta=\left(1+\left|x_{0}\right|\right)^{-b / \gamma^{\prime}}$. Let

$$
v_{n}(y):=\delta^{\frac{2-\gamma}{\gamma-1}} u_{n}\left(x_{0}+\delta y\right) \quad \text { on } \mathbb{R}^{N} .
$$

Then, $v_{n}$ solves

$$
-\Delta v_{n}+C_{H}\left|\nabla v_{n}\right|^{\gamma}=\delta^{\gamma^{\prime}}\left(F_{n}\left(x_{0}+\delta y\right)-f_{n}\left(x_{0}+\delta y\right)-\lambda_{n}\right) .
$$

Since $\delta \leq 1$,

$$
\delta^{\gamma^{\prime}}\left|F_{n}\left(x_{0}+\delta y\right)-f_{n}\left(x_{0}+\delta y\right)-\lambda_{n}\right| \leq \frac{C_{F}\left(3+\left|x_{0}\right|\right)^{b}+c_{f}+\lambda}{\left(1+\left|x_{0}\right|\right)^{b}} \leq C_{1}
$$

for all $y \in B_{2}(0)$ by (2.4) and the bound on $f_{n}$.

Therefore, by the first claim,

$$
\left\|\nabla v_{n}\right\|_{L^{\infty}\left(B_{1}(0)\right)} \leq \widetilde{C}
$$

for all $n$. In particular, choosing $y=0$,

$$
\left|\nabla u_{n}\left(x_{0}\right)\right|=\delta^{-\frac{1}{\gamma-1}}\left|\nabla v_{n}(0)\right| \leq \widetilde{C}\left(1+\left|x_{0}\right|\right)^{b / \gamma},
$$

and the desired estimate follows.

Moreover, we prove the following a priori estimates on bounded from below solutions to (2.3).

Theorem 2.6. Let $u_{n} \in C^{2}\left(\mathbb{R}^{N}\right)$ be a family of uniformly bounded from below classical solutions to (2.3), that is for which there exists $C>0$ such that $u_{n} \geq-C$ for every $n$.

If $b=0$ in (2.4), we moreover assume that there exists $\delta>0$ and $R>0$ independent of $n$ such that

$$
F_{n}(x)-f_{n}(x)-\lambda_{n}>\delta>0, \quad \text { for all }|x|>R .
$$

Then there exists $C>0$ such that

$$
u_{n}(x) \geq C|x|^{1+\frac{b}{\gamma}}-C^{-1}, \quad \forall n \in \mathbb{N}, x \in \mathbb{R}^{N},
$$

where $b \geq 0$ is the growth power appearing in (2.4) and $\gamma$ is the growth power appearing in (1.2).

Proof. The proof is based on the same argument as in [4. Proposition 3.4], we sketch it briefly for completeness. Since $u_{n}$ is bounded from below we can assume $u_{n} \geq 0$, up to addition of constant $C$ (without changing the equation).

We assume by contradiction that (2.8) does not hold. Then there exist sequences $x_{l}$ and $u_{n_{l}}$, such that $\left|x_{l}\right|>2 R,\left|x_{l}\right| \rightarrow+\infty$, and $\frac{u_{n_{l}}\left(x_{l}\right)}{\left|x_{l}\right|^{1+\frac{b}{\gamma}}} \rightarrow 0$. Let $a_{l}=\frac{\left|x_{l}\right|}{2}$ and we define the function

$$
v^{l}(x)=\frac{1}{a_{l}^{1+\frac{b}{\gamma}}} u_{n_{l}}\left(x_{l}+a_{l} x\right) .
$$


By Theorem 2.5, we get that $\left|\nabla u_{n_{l}}(x)\right| \leq K(1+|x|)^{\frac{b}{\gamma}}$. Therefore, $v^{l},\left|\nabla v^{l}\right|$ are uniformly bounded. Moreover, $v^{l}$ is a solution to

$$
-a_{l}^{\frac{b}{\gamma}-1} \Delta v^{l}+H_{n_{l}}\left(a_{l}^{\frac{b}{\gamma}} \nabla v^{l}\right)+\lambda_{n_{l}}=F_{n_{l}}\left(x_{l}+a_{l} x\right)-f_{n_{l}}\left(x_{l}+a_{l} x\right) .
$$

In particular, recalling (1.2), we get that $v^{l}$ is a supersolution to

$$
-a_{l}^{\frac{b}{\gamma}-1-b} \Delta v^{l}+C_{H}\left|\nabla v^{l}\right|^{\gamma} \geq a_{l}^{-b}\left(-\lambda_{n_{l}}+F_{n_{l}}\left(x_{l}+a_{l} x\right)-f_{n_{l}}\left(x_{l}+a_{l} x\right)\right) .
$$

Note that, for every $l$ sufficiently large, by (2.4) and by (2.7) (in the case $b=0$ ) the right hand side of the equation

$$
a_{l}^{-b}\left(-\lambda_{n_{l}}+F_{n_{l}}\left(x_{l}+a_{l} x\right)-f_{n_{l}}\left(x_{l}+a_{l} x\right)\right)>0
$$

for $x$ such that $|x| \leq 1$.

Moreover, passing eventually to a subsequence, we get that $v^{l} \rightarrow v$ locally uniformly in $n$ and $a_{l}^{\frac{b}{\gamma}-1-b} \rightarrow 0$. So $v$ is a supersolution to $C_{H}|\nabla v|^{\gamma} \geq \delta>0$ in $B(0,1)$ with homogeneous boundary conditions (since $v \geq 0$ ). By comparison, recalling the explicit formula of the solution to the eikonal equation $|\nabla f|^{\gamma}=C$ in $B(0,1)$ with homogeneous boundary conditions, we conclude that $v(x) \geq C^{\frac{1}{\gamma}}(1-|x|)$ for all $x$ such that $|x| \leq 1$. Moreover, by uniform convergence, we get that, eventually enlarging $C$ and taking $l$ sufficiently large, $v^{l}(x) \geq C^{\frac{1}{\gamma}}(1-|x|)$ for all $x$ with $|x| \leq 1$, in particular $v^{l}(0) \geq C^{\frac{1}{\gamma}}$. Recalling the definition of $v^{l}$, we get that $v^{l}(0) \rightarrow 0$, which yields a contradiction.

Define

$$
\bar{\lambda}_{n}:=\sup \left\{\lambda \in \mathbb{R}:(2.3) \text { has a solution } u_{n} \in C^{2}\left(\mathbb{R}^{N}\right)\right\} .
$$

Theorem 2.7. Assume that for every $n$ the function $F_{n}-f_{n}$ is bounded from below uniformly in $n$.

(i) $\bar{\lambda}_{n}<\infty$, for every $n$, and there exists, for every $n$, a solution $u_{n} \in C^{2}\left(\mathbb{R}^{N}\right)$ to (2.3) with $\lambda_{n}=\bar{\lambda}_{n}$. Moreover

$$
\bar{\lambda}_{n}:=\sup \left\{\lambda \in \mathbb{R}:(2.3) \text { has a subsolution } u_{n} \in C^{2}\left(\mathbb{R}^{N}\right)\right\} .
$$

(ii) If $F_{n}$ satisfies (2.4), with $b>0$, then, for every $n$, the solution $u_{n}$ to (2.3) with $\lambda_{n}=\bar{\lambda}_{n}$ is unique up to addition of constants and satisfies (2.8).

(iii) If $F_{n} \equiv 0$, and there exists $\delta>0$ independent of $n$ such that

$$
\limsup _{|x| \rightarrow+\infty} f_{n}(x)+\bar{\lambda}_{n}<-\delta<0,
$$

then for every $n$ there exists a solution to (2.3) with $\lambda_{n}=\bar{\lambda}_{n}$ which satisfies (2.8) with $b=0$.

Proof. (i). The proof of this result can be obtained by a straightforward adaptation of the proof of Theorem 2.1 in [4, using the a priori estimates on the gradient given in Theorem 2.5. Observe that actually in 4 it is required a stronger assumption on the regularity of $F_{n}-f_{n}$, in particular local Lipschitz continuity. This assumption is used to derive a priori estimates on the gradient of solutions by using the so called Bernstein method (see Appendix A in [4), which depends also on the $L^{\infty}$ norm of $\nabla\left(F_{n}-f_{n}\right)$. In our case we can weaken this assumption to just Hölder continuity (so still ensuring classical elliptic regularity) since we are using a priori estimates on the gradient given in Theorem 2.5. which depends only on the $L^{\infty}$ norm of $F_{n}-f_{n}$, and are obtained in [21] by the so called integral Bernstein method.

(ii). For the proof we refer to [19] (see also [4] and [11]). In particular in [19, it is proved that $u_{n}$ is bounded from below. By looking at the proof, it is easy to check that, due to the uniformity in $n$ of the norms of coefficients, the bound can be taken independent of $n$, and by Theorem 2.6 we get the estimate on the growth. 
(iii). By adapting the argument in [4, Theorem 2.6], we get that there exists a bounded from below solution to (2.3) with $\lambda_{n}=\bar{\lambda}_{n}$, with bound uniform in $n$. Then using Theorem 2.6, we get the estimate on the growth. We give a brief sketch of the proof of the existence of a bounded from below solution. For every $R>0$, we consider the ergodic problem

$$
\begin{cases}-\Delta u_{n}^{R}+H_{n}\left(\nabla u_{n}^{R}\right)+\lambda_{n}^{R}=-f & |x|<R \\ u_{n}^{R}(x) \rightarrow+\infty & |x| \rightarrow R .\end{cases}
$$

Using the result in [5], we get that for every $R>0$ there exists a unique $\lambda_{n}^{R}$ and a unique up to addition of constant solution $u_{n}^{R} \in C^{2}\left(B_{R}\right)$.

First of all we claim that $\lim _{R} \lambda_{n}^{R}=\bar{\lambda}_{n}$. It is easy to check that if $R^{\prime}>R$, then $\lambda_{n}^{R^{\prime}} \leq \lambda_{n}^{R}$, and moreover that $\lambda_{n}^{R} \geq \bar{\lambda}_{n}$. So, the sequence $\lambda_{n}^{R}$ is converging as $R \rightarrow+\infty$ to some $\lambda_{n}^{\star} \geq \bar{\lambda}_{n}$. Moreover, by the same argument as in Theorem 2.5. we get that for every compact $K \subset \mathbb{R}^{N}$, there exists a constant $C>0$ such that $\left|\nabla u_{n}^{R}\right| \leq C$ in $K$ for every $R$ sufficiently large and for all $n$. Without loss of generality we can assume that $u_{n}^{R}(0)=0$ for every $R$. So, using the gradient bound, and elliptic regularity, we conclude that $u_{n}^{R}$ is bounded in $C^{2}(K)$ by some constant independent of $R$. Hence, by Ascoli-Arzelà Theorem, and via a diagonalization procedure, we get that $u_{n}^{R}$ converges locally in $\mathbb{R}^{N}$, with $u_{n} \in C^{2}\left(\mathbb{R}^{N}\right)$. Moreover, $u_{n}$ is a solution to (2.3), with $\lambda=\lambda_{n}^{\star}$. Recalling the characterization of $\bar{\lambda}_{n}$ and the fact that $\lambda_{n}^{\star} \geq \bar{\lambda}_{n}$, we conclude that $\lambda_{n}^{\star}=\bar{\lambda}_{n}$.

Then, we consider $x_{n}^{R} \in B_{R}$ such that $u_{n}^{R}\left(x_{n}^{R}\right)=\min _{|x| \leq R} u_{n}^{R}$. Recalling that $u_{n}^{R}$ is a solution to (2.10), we get by computing the equation at $x_{n}^{R}$ and by recalling that $H_{n}(0) \leq 0$, that

$$
\lambda_{n}^{R}+f\left(x_{n}^{R}\right) \geq H_{n}(0)+\lambda_{n}^{R}+f\left(x_{n}^{R}\right) \geq 0 .
$$

Using condition (2.9), and recalling that $\lambda_{n}^{R} \rightarrow \bar{\lambda}_{n}$, we get that there exists a compact set $K$ (independent of $R$ and of $n$ ) and $R_{0}>0$ such that for all $R>R_{0}, x_{n}^{R} \in K$.

Recalling that $u_{n}^{R}(0)=0$ and $\left|\nabla u_{n}^{R}\right| \leq C$ in $K$ with $C$ independent of $n, R$, we conclude that $u_{n}^{R}\left(x_{R}\right) \geq-C$ for some constant $C$ independent of $n, R$. But, this implies, since $u_{n}^{R}(x) \geq u_{n}^{R}\left(x_{n}^{R}\right)$ for every $R$, that passing to the limit $u_{n}(x) \geq-C$, with $C$ independent of $n$.

\subsection{A priori estimates for the Kolmogorov equation}

In this section we provide general a priori estimates for couples $(m, w) \in\left(L^{1}\left(\mathbb{R}^{N}\right) \cap W^{1, q}\left(\mathbb{R}^{N}\right)\right) \times$ $L^{1}\left(\mathbb{R}^{N}\right)$ such that $\int_{\mathbb{R}^{N}} m(x)=M$ and $-\varepsilon \Delta m+\operatorname{div} w=0$ where

$$
q= \begin{cases}\gamma^{\prime} & \gamma^{\prime} \geq N \\ \frac{N}{N-\gamma^{\prime}+1} & \gamma^{\prime}<N .\end{cases}
$$

Lemma 2.8. Let $\beta \leq \frac{N q}{N-q}$, for $q<N$, and $\beta<+\infty$ for $q \geq N$. We define $1 \leq r \leq \beta$ as follows

$$
\frac{1}{r}=\frac{1}{\gamma^{\prime}}+\left(1-\frac{1}{\gamma^{\prime}}\right) \frac{1}{\beta} .
$$

Then, there exists a constant $C$, depending only on $N$ and $\beta$, such that

$$
\begin{aligned}
\|m\|_{W^{1, r}\left(\mathbb{R}^{N}\right)} & \leq C\left(\frac{1}{\varepsilon^{\gamma^{\prime}}} \int_{\mathbb{R}^{N}} m\left|\frac{w}{m}\right|^{\gamma^{\prime}} d x+M\right)^{\frac{1}{\gamma^{\prime}}}\|m\|_{L^{\beta}\left(\mathbb{R}^{N}\right)}^{\frac{1}{\gamma}} \\
& \leq C\left(\frac{C_{L}}{\varepsilon^{\gamma^{\prime}}} \int_{\mathbb{R}^{N}} m L\left(-\frac{w}{m}\right) d x+M\right)^{\frac{1}{\gamma^{\prime}}}\|m\|_{L^{\beta}\left(\mathbb{R}^{N}\right)}^{\frac{1}{\gamma}},
\end{aligned}
$$

where $C_{L}=C_{L}\left(C_{H}, \gamma\right)$ is the constant appearing in Proposition 2.1.

We now assume that

$$
1<\beta<1+\frac{\gamma^{\prime}}{N}
$$


Then, there exists $\delta>0$ such that

$$
\|m\|_{L^{\beta}\left(\mathbb{R}^{N}\right)}^{(1+\delta) \beta} \leq C \frac{1}{\varepsilon^{\gamma^{\prime}}} M^{(1+\delta) \beta-1}\left(\int_{\mathbb{R}^{N}} m\left|\frac{w}{m}\right|^{\gamma^{\prime}} d x\right) \leq C C_{L} \frac{1}{\varepsilon^{\gamma^{\prime}}} M^{(1+\delta) \beta-1} \int_{\mathbb{R}^{N}} m L\left(-\frac{w}{m}\right) d x,
$$

where the constant $C$ depends only on $\gamma, N$, and $\beta$.

Proof. Since $m \in W^{1, q}\left(\mathbb{R}^{N}\right)$, by Sobolev embedding and interpolation, we get that $m \in L^{\beta}\left(\mathbb{R}^{N}\right)$. Using $-\varepsilon \Delta m+\operatorname{div} w=0$, we get for all $\varphi \in C_{0}^{\infty}\left(\mathbb{R}^{N}\right)$,

$$
\varepsilon \int_{\mathbb{R}^{N}} \nabla m \cdot \nabla \varphi d x=\int_{\mathbb{R}^{N}} w \cdot \nabla \varphi d x
$$

Using Holder inequality, recalling (2.12), we obtain

$$
\begin{aligned}
\left|\frac{1}{\varepsilon} \int_{\mathbb{R}^{N}} w \cdot \nabla \varphi d x\right| \leq \int_{\mathbb{R}^{N}} \frac{1}{\varepsilon}\left|\frac{w}{m}\right| m^{\frac{1}{\gamma^{\prime}}} m^{1-\frac{1}{\gamma^{\prime}}}|\nabla \varphi| d x & \\
& \leq\left(\frac{1}{\varepsilon^{\gamma^{\prime}}} \int_{\mathbb{R}^{N}} m\left|\frac{w}{m}\right|^{\gamma^{\prime}} d x\right)^{\frac{1}{\gamma^{\prime}}}\|m\|_{L^{\beta}\left(\mathbb{R}^{N}\right)}^{\frac{1}{\gamma}}\|\nabla \varphi\|_{L^{r^{\prime}\left(\mathbb{R}^{N}\right)} .}
\end{aligned}
$$

Therefore, we get that for all $\varphi \in C_{0}^{\infty}\left(\mathbb{R}^{N}\right)$,

$$
\left|\int_{\mathbb{R}^{N}} \nabla m \cdot \nabla \varphi d x\right| \leq\left(\frac{1}{\varepsilon^{\gamma^{\prime}}} \int_{\mathbb{R}^{N}} m\left|\frac{w}{m}\right|^{\gamma^{\prime}} d x\right)^{\frac{1}{\gamma^{\prime}}}\|m\|_{L^{\beta}\left(\mathbb{R}^{N}\right)}^{\frac{1}{\gamma}}\|\nabla \varphi\|_{r^{\prime}} .
$$

We apply then Proposition 2.4 and we obtain that $m \in W^{1, r}\left(\mathbb{R}^{N}\right)$ and that there exists a constant $C$, depending only on $r$, such that

$$
\|\nabla m\|_{L^{r}\left(\mathbb{R}^{N}\right)} \leq C\left(\frac{1}{\varepsilon^{\gamma^{\prime}}} \int_{\mathbb{R}^{N}} m\left|\frac{w}{m}\right|^{\gamma^{\prime}} d x\right)^{\frac{1}{\gamma^{\prime}}}\|m\|_{L^{\beta}\left(\mathbb{R}^{N}\right)}^{\frac{1}{\gamma}} .
$$

From this inequality, using Proposition 2.1 and recalling that by interpolation, since $\|m\|_{L^{1}\left(\mathbb{R}^{N}\right)}=$ $M,\|m\|_{L^{r}\left(\mathbb{R}^{N}\right)} \leq\|m\|_{L^{\beta}\left(\mathbb{R}^{N}\right)}^{\frac{1}{\gamma}} M^{\frac{1}{\gamma^{\prime}}}$, we conclude the desired inequality (2.13).

Now we fix $\eta$ such that

$$
\frac{1}{\eta}=\left(\frac{1}{r}-\frac{1}{N}\right) \frac{N}{N+1}+1-\frac{N}{N+1}=\frac{N}{N+1} \frac{1}{r}
$$

Note that, by a simple computation using (2.12), we get $\frac{1}{\eta}-\frac{1}{\beta}=\frac{N}{N+1} \frac{1}{\beta \gamma^{\prime}}\left(\beta-1-\frac{\gamma^{\prime}}{N}\right)$, therefore, by (2.14), we conclude that that $\eta>\beta$. By Gagliardo Nirenberg inequality, and recalling that $\|m\|_{1}=M$, we get

$$
\|m\|_{L^{\eta}\left(\mathbb{R}^{N}\right)} \leq C\|\nabla m\|_{L^{r}\left(\mathbb{R}^{N}\right)}^{\frac{N}{N+1}} M^{\frac{1}{N+1}} .
$$

Since $\eta>\beta$, by interpolation we get that there exists $\theta>1$ such that $\|m\|_{L^{\beta}\left(\mathbb{R}^{N}\right)}^{\theta} \leq\|m\|_{L^{\eta}\left(\mathbb{R}^{N}\right)} M^{\theta-1}$. Actually

$$
\frac{1}{\theta}=\left(1-\frac{1}{\beta}\right)(N+1) \frac{1}{1+N\left(1-\frac{1}{\beta}\right)\left(1-\frac{1}{\gamma^{\prime}}\right)} .
$$

So, we substitute in (2.17) and (2.16) and we get, elevating both terms to $\gamma^{\prime} \frac{N+1}{N}$,

$$
\|m\|_{L^{\beta}\left(\mathbb{R}^{N}\right)}^{\theta \gamma^{\prime}} \leq C \frac{1}{\varepsilon^{\gamma^{\prime}}} M^{\gamma^{\prime}\left(\theta \frac{N+1}{N}-1\right)}\left(\int_{\mathbb{R}^{N}} m\left|\frac{w}{m}\right|^{\gamma^{\prime}} d x\right)\|m\|_{L^{\beta}\left(\mathbb{R}^{N}\right)}^{\frac{\gamma^{\prime}}{\gamma}} .
$$


Now, since $\theta>1$, by (2.14), we get

$$
\theta \gamma^{\prime} \frac{N+1}{N}-\frac{\gamma^{\prime}}{\gamma}=\frac{\beta \gamma^{\prime}}{N(\beta-1)}=\beta+\frac{\beta}{\beta-1}\left[\frac{\gamma^{\prime}}{N}+1-\beta\right]>0 .
$$

Therefore we deduce (2.15) from (2.18) with

$$
\delta=\frac{1}{\beta-1}\left[\frac{\gamma^{\prime}}{N}+1-\beta\right] .
$$

Corollary 2.9. For every $r<q$, there exists $C>0$ depending on $N, \gamma^{\prime}$ and $r$ such that

$$
\|m\|_{W^{1, r}\left(\mathbb{R}^{N}\right)} \leq \frac{C}{\varepsilon^{\gamma^{\prime}}}\left(C_{L} \int_{\mathbb{R}^{N}} m L\left(-\frac{w}{m}\right) d x+\varepsilon^{\gamma^{\prime}} M\right) .
$$

Moreover, if $\gamma^{\prime}>N($ so $q>N)$, then $m \in C^{0, \theta}\left(\mathbb{R}^{N}\right)$ and

$$
\|m\|_{C^{0, \theta}\left(\mathbb{R}^{N}\right)} \leq \frac{C}{\varepsilon^{\gamma^{\prime}}}\left(C_{L} \int_{\mathbb{R}^{N}} m L\left(-\frac{w}{m}\right) d x+\varepsilon^{\gamma^{\prime}} M\right) .
$$

Proof. For $q \geq N$ (equivalently $\gamma^{\prime} \geq N$ ), we fix $r<q$ and we choose $\beta$ which satisfies (2.12) for such $r$. By Sobolev embedding theorem, $W^{1, r}\left(\mathbb{R}^{N}\right)$ is continuously embedded in $L^{\beta}\left(\mathbb{R}^{N}\right)$. So, there exists $C$ depending on $N$ and $r$ such that $\|m\|_{L^{\beta}\left(\mathbb{R}^{N}\right)} \leq C\|m\|_{W^{1, r}\left(\mathbb{R}^{N}\right)}$. Using inequality (2.13), we get

$$
\|m\|_{L^{\beta}\left(\mathbb{R}^{N}\right)} \leq \frac{C}{\varepsilon^{\gamma^{\prime}}}\left(\int_{\mathbb{R}^{N}} m\left|\frac{w}{m}\right|^{\gamma^{\prime}} d x+\varepsilon^{\gamma^{\prime}} M\right) .
$$

If we substitute again in (2.13) we get

$$
\|m\|_{W^{1, r}\left(\mathbb{R}^{N}\right)} \leq \frac{C}{\varepsilon^{\gamma^{\prime}}}\left(\int_{\mathbb{R}^{N}} m\left|\frac{w}{m}\right|^{\gamma^{\prime}} d x+\varepsilon^{\gamma^{\prime}} M\right) .
$$

In particular for $q>N$, we can choose $r>N$ and by Sobolev embedding theorem we get that there exists $\theta=1-\frac{N}{r}$ and a constant $C>0$ depending on $N$ and $r$ such that

$$
\begin{aligned}
\|m\|_{C^{0, \theta}\left(\mathbb{R}^{N}\right)} & \leq \frac{C}{\varepsilon^{\gamma^{\prime}}}\left(\int_{\mathbb{R}^{N}} m\left|\frac{w}{m}\right|^{\gamma^{\prime}} d x+\varepsilon^{\gamma^{\prime}} M\right) \\
& \leq \frac{C}{\varepsilon^{\gamma^{\prime}}}\left(C_{L} \int_{\mathbb{R}^{N}} m L\left(-\frac{w}{m}\right) d x+\varepsilon^{\gamma^{\prime}} M\right) .
\end{aligned}
$$

For $q<N$, we fix $r<q$, and choose the corresponding $\beta$ in (2.12), that satisfies $\beta<\frac{N}{N-\gamma^{\prime}}$. Hence we conclude again from inequality (2.13).

\section{Regularization procedure and existence of approximate solutions for $\varepsilon>0$}

\subsection{The regularized problem}

We consider the following approximation of the system (1.1),

$$
\left\{\begin{array}{l}
-\varepsilon \Delta u+H(\nabla u)+\lambda=f_{k}[m](x)+V(x), \\
-\varepsilon \Delta m-\operatorname{div}(m \nabla H(\nabla u))=0, \\
\int_{\mathbb{R}^{N}} m d x=M,
\end{array}\right.
$$


where

$$
f_{k}[m](x)=f\left(m \star \chi_{k}\right) \star \chi_{k}(x)=\int_{\mathbb{R}^{N}} \chi_{k}(x-y) f\left(\int_{\mathbb{R}^{N}} m(z) \chi_{k}(y-z) d z\right) d y
$$

and $\chi_{k}$, for $k>0$, is a sequence of standard symmetric mollifiers approximating the unit as $k \rightarrow \infty$.

We observe that $f_{k}[m](x)$ is the $L^{2}$-gradient of a $C^{1}$ potential $F_{k}: L^{1}\left(\mathbb{R}^{N}\right) \rightarrow \mathbb{R}$, defined as follows

$$
F_{k}[m]:=\int_{\mathbb{R}^{N}} F\left(m \star \chi_{k}(x)\right) d x
$$

where $F(m)=\int_{0}^{m} f(n) d n$ for $m \geq 0$ and $F(m)=0$ for $m \leq 0$. Note that using Jensen inequality and (1.3), we get that for all $m \in L^{1}\left(\mathbb{R}^{N}\right)$ such that $m \geq 0$, and $\int_{\mathbb{R}^{N}} m(x) d x=M$,

$$
-\frac{C_{f}}{\alpha+1} \int_{\mathbb{R}^{N}} m^{\alpha+1}(x) d x-K M \leq F_{k}[m] \leq-\frac{C_{f}}{\alpha+1} \int_{\mathbb{R}^{N}}\left(m \star \chi_{k}(x)\right)^{\alpha+1} d x+K M .
$$

In order to construct solutions to the system, we follow a variational approach and we associate to (3.1) a energy, as already described in the introduction. We define the energy

$$
\mathcal{E}_{k}(m, w):= \begin{cases}\int_{\mathbb{R}^{N}} m L\left(-\frac{w}{m}\right)+V(x) m d x+F_{k}[m] & \text { if }(m, w) \in \mathcal{K}_{\varepsilon, M}, \\ +\infty & \text { otherwise }\end{cases}
$$

where $\mathcal{K}_{\varepsilon, M}$ is defined in (1.8) and $L$ is defined in (1.7). We recall that the exponent $q$ appearing in the definition of $\mathcal{K}_{\varepsilon, M}$ is

$$
q= \begin{cases}\frac{N}{N-\gamma^{\prime}+1} & \gamma^{\prime} \leq N \\ \gamma^{\prime} & \gamma^{\prime}>N\end{cases}
$$

Therefore, $q \leq \gamma^{\prime}$. Observe that, if $q<N, q^{*}=\frac{q N}{N-q}=\frac{N}{N-\gamma^{\prime}}$, and that $q^{*}>1+\frac{\gamma^{\prime}}{N}>1+\alpha$ by (1.4). If $q=\gamma^{\prime} \geq N$, then we let $q^{*}=+\infty$.

\subsection{A priori estimates and energy bounds}

In this section, we provide bounds from below for the energy $\mathcal{E}_{k}$, assuring in particular that the minimum problem is well defined.

Lemma 3.1. Let $(m, w) \in \mathcal{K}_{\varepsilon, M}$. Then

$$
\mathcal{E}_{k}(m, w) \geq-K-C \varepsilon^{-\frac{\gamma^{\prime} \alpha N}{\gamma^{\prime}-\alpha N}}
$$

where $C, K>0$ are constants depending only on $N, M, C_{L}, \gamma, \alpha, M$.

In particular there exists finite

$$
e_{k, \varepsilon}(M)=\inf _{(m, w) \in \mathcal{K}_{\varepsilon, M}} \mathcal{E}_{k}(m, w) .
$$

Proof. Recalling that $V \geq 0$, estimate (3.4) and applying (2.15) with $\alpha=\beta-1$, we get

$$
\begin{aligned}
\mathcal{E}_{k}(m, w) & \geq \int_{\mathbb{R}^{N}} m L\left(-\frac{w}{m}\right) d x-\frac{C_{f}}{\alpha+1} \int_{\mathbb{R}^{N}} m^{\alpha+1} d x-K M \\
& \geq C \varepsilon^{\gamma^{\prime}} M^{1-(1+\delta)(1+\alpha)}\|m\|_{L^{\alpha+1}}^{(1+\alpha)(1+\delta)}-\frac{1}{\alpha+1}\|m\|_{L^{\alpha+1}}^{(1+\alpha)}-K M \\
& \geq-C \delta \varepsilon^{-\frac{\gamma^{\prime}}{\delta}}\left(\frac{1}{(\delta+1)(\alpha+1)}\right)^{1+\frac{1}{\delta}}-K M
\end{aligned}
$$

where $C$ is a constant depending only on $N, M, C_{L}, \gamma, \alpha$ and

$$
\delta=\frac{1}{\alpha}\left[\frac{\gamma^{\prime}}{N}-\alpha\right] .
$$


Therefore, substituting in the energy, we get

$$
\mathcal{E}_{k}(m, w) \geq-C \frac{\left(\gamma^{\prime}-\alpha N\right)}{\alpha N} \varepsilon^{-\frac{\gamma^{\prime} \alpha N}{\gamma^{\prime}-\alpha N}}\left(\frac{\alpha N}{\gamma^{\prime}(\alpha+1)}\right)^{\frac{\gamma^{\prime}}{\gamma^{\prime}-\alpha N}}-K M,
$$

which gives the desired inequality.

We get also a priori bounds on minimizers and minimizing sequences.

Proposition 3.2. Let $(m, w) \in \mathcal{K}_{\varepsilon, M}$ such that $e_{k, \varepsilon}(M) \geq \mathcal{E}_{k}(m, w)-\eta$, for some positive $\eta$. Then

$$
\begin{aligned}
\int_{\mathbb{R}^{N}} m\left|\frac{w}{m}\right|^{\gamma^{\prime}} d x & \leq C \varepsilon^{-\frac{\gamma^{\prime} N \alpha}{\gamma^{\prime}-N \alpha}}+K, \\
\|m\|_{L^{\alpha+1}\left(\mathbb{R}^{N}\right)}^{\alpha+1} & \leq C \varepsilon^{-\frac{\gamma^{\prime} N \alpha}{\gamma^{\prime}-N \alpha}}+K,
\end{aligned}
$$

for some $C, K$ positive constants which depends only on $\alpha, N, V, C_{L}$.

Proof. First of all we observe that there exists $C \geq 0$ depending on $M, C_{L}, C_{V}$ such that

$$
e_{k, \varepsilon}(M) \leq C .
$$

Let $m=c e^{-|x|}$, where $c$ is chosen to have $\int_{\mathbb{R}^{n}} m d x=M$, and $w=\varepsilon \nabla m$, so that $(m, w) \in \mathcal{K}_{\varepsilon, M}$. By assumption (1.5), we get that $\int_{\mathbb{R}^{n}} m V(x) d x \leq C$ for some constant $C>0$, by (3.4) that $F_{k}[m] \leq K M$ and by the properties of $L$ in Proposition 2.1, we have that $\int_{\mathbb{R}^{n}} m L(-w / m) d x \leq$ $\left(\frac{\varepsilon^{\gamma^{\prime}}}{c^{\gamma^{\prime}}}+C_{L}\right) M$. So, in conclusion $e_{k, \varepsilon}(M) \leq \mathcal{E}_{k}(m, w) \leq C$ as required.

Note that if $(m, w) \in \mathcal{K}_{\varepsilon, M}$, and $e_{\varepsilon}(M) \geq \mathcal{E}(m, w)-\eta$, for some positive $\eta$, then, by (3.4), by the fact that $V \geq 0$, and by the properties of $L$ in Proposition 2.1] we get

$$
C+\eta \geq e_{\varepsilon}(M)+\eta \geq \mathcal{E}_{k}(m, w) \geq \int_{\mathbb{R}^{N}} m\left|\frac{w}{m}\right|^{\gamma^{\prime}}-\frac{C_{f}}{\alpha+1} m^{\alpha+1} d x-K M .
$$

We apply (2.15) with $\alpha=\beta-1$, and we obtain

$$
\begin{aligned}
C+\eta+K M \geq \int_{\mathbb{R}^{N}} m\left|\frac{w}{m}\right|^{\gamma^{\prime}}-\frac{C_{f}}{\alpha+1} & m^{\alpha+1} d x \\
& \geq C \varepsilon^{\gamma^{\prime}} M^{1-(1+\delta)(1+\alpha)}\|m\|_{L^{\alpha+1}}^{(1+\alpha)(1+\delta)}-\frac{C_{f}}{\alpha+1}\|m\|_{L^{\alpha+1}}^{(1+\alpha)} .
\end{aligned}
$$

Recall that $\delta+1=\frac{\gamma^{\prime}}{\alpha N}$ (can be computed using (2.19) $)$, so $\frac{\gamma^{\prime}}{\delta}=\frac{\gamma^{\prime} N \alpha}{\gamma^{\prime}-N \alpha}$. Note that if we choose $A$ sufficiently large (depending on $\delta, M, C_{f}, C_{L}$ ), we get that

$$
C \varepsilon^{\gamma^{\prime}} M^{1-(1+\delta)(1+\alpha)}\left(\varepsilon^{-\frac{\gamma^{\prime}}{\delta}} A\right)^{1+\delta}-\frac{C_{f}}{\alpha+1}\left(\varepsilon^{-\frac{\gamma^{\prime}}{\delta}} A\right) \geq C+\eta+K M,
$$

from which we conclude that $\|m\|_{L^{\alpha+1}}^{(1+\alpha)} \leq \varepsilon^{-\frac{\gamma^{\prime}}{\delta}} A$, and so estimate (3.9) holds. Estimate (3.8) comes from (3.9) and (3.11).

\subsection{Existence of a solution}

We are now in the position to show existence of minimizers of the energy $\mathcal{E}_{k}$ in the class $\mathcal{K}_{\varepsilon, M}$ for every $\varepsilon, M>0$. 
Proposition 3.3. For every $\varepsilon>0$ and $M>0$, there exists a minimizer $\left(m_{k}, w_{k}\right) \in \mathcal{K}_{\varepsilon, M}$ of $\mathcal{E}_{k}$, that is

$$
\mathcal{E}_{k}\left(m_{k}, w_{k}\right)=\inf _{(m, w) \in \mathcal{K}_{\varepsilon, M}} \mathcal{E}_{k}(m, w) .
$$

Moreover, for every minimizer $\left(m_{k}, w_{k}\right) \in \mathcal{K}_{\varepsilon, M}$ of $\mathcal{E}_{k}$, there holds

$$
m_{k}(1+|x|)^{b} \in L^{1}\left(\mathbb{R}^{N}\right), \quad w_{k}(1+|x|)^{b / \gamma} \in L^{1}\left(\mathbb{R}^{N}\right),
$$

and there exist constants $C>0$ and $K$, independent of $\varepsilon$ and $k$, such that

$$
\int_{\mathbb{R}^{N}} m_{k}\left|\frac{w_{k}}{m_{k}}\right|^{\gamma^{\prime}} d x+\int_{R^{N}} m_{k} V(x) d x+\left\|m_{k}\right\|_{L^{\alpha+1}\left(\mathbb{R}^{N}\right)}^{\alpha+1} \leq C \varepsilon^{-\frac{\gamma^{\prime} \alpha N}{\gamma^{\prime}-N \alpha}}+K
$$

Proof. Let $\left(m_{n}, w_{n}\right) \in \mathcal{K}_{\varepsilon, M}$ be a minimizing sequence, that is $\mathcal{E}_{k}\left(m_{n}, w_{n}\right) \rightarrow e_{k, \varepsilon}(M)$. This implies that, choosing $n$ sufficiently large, $\mathcal{E}_{k}\left(m_{n}, w_{n}\right) \leq e_{\varepsilon}(M)+1$. From this and (3.4) we get

$$
\begin{array}{r}
\int_{\mathbb{R}^{N}} m_{n} L\left(-\frac{w_{n}}{m_{n}}\right) d x+\int_{\mathbb{R}^{N}} V(x) m_{n} d x \leq \mathcal{E}_{k}\left(m_{n}, w_{n}\right)+\frac{C_{f}}{\alpha+1} \int_{\mathbb{R}^{N}} m_{n}^{\alpha+1} d x+K M \\
\leq e_{k, \varepsilon}(M)+1+\frac{C_{f}}{\alpha+1} \int_{\mathbb{R}^{N}} m_{n}^{\alpha+1}+K M
\end{array}
$$

By Proposition 3.2, we get that

$$
\left\|m_{n}\right\|_{L^{\alpha+1}}+\int_{\mathbb{R}^{N}} m_{n}^{1-\gamma^{\prime}}\left|w_{n}\right|^{\gamma^{\prime}} d x \leq C \varepsilon^{-\frac{\gamma^{\prime} \alpha N}{\gamma^{\prime}-\alpha N}}+K
$$

We conclude also that

$$
\int_{\mathbb{R}^{N}} V(x) m_{n}(x) d x \leq C \varepsilon^{-\frac{\gamma^{\prime} \alpha N}{\gamma^{\prime}-\alpha N}}+K
$$

for some $C, K>0$. These estimates will imply (3.13), after passing to the limit, using Fatou lemma.

Moreover, by Corollary 2.9, we have that there exists $C_{\varepsilon}>0$ depending on $\varepsilon$ such that for all $r<q$,

$$
\left\|m_{n}\right\|_{W^{1, r}\left(\mathbb{R}^{N}\right)} \leq C_{\varepsilon} .
$$

Moreover, due to Sobolev embeddings, we get that for all $s<q^{*}$, then $\left\|m_{n}\right\|_{L^{s}\left(\mathbb{R}^{N}\right)} \leq C_{\varepsilon}$. In addition, by applying Holder inequality, we get that there exists $C>0$

$$
\int_{\mathbb{R}^{N}}\left|w_{n}\right|^{\frac{\gamma^{\prime} \alpha+\gamma^{\prime}}{\gamma^{\prime}+\alpha}} d x \leq C\left(\int_{\mathbb{R}^{N}} m_{n}^{1-\gamma^{\prime}}\left|w_{n}\right|^{\gamma^{\prime}} d x\right)^{\frac{\alpha+1}{\gamma^{\prime}+\alpha}}\left\|m_{n}\right\|_{L^{\alpha+1}\left(\mathbb{R}^{N}\right)}^{\frac{\gamma^{\prime}-1}{(\alpha+1)\left(\gamma^{\prime}+\alpha\right.}} .
$$

By these estimates and Sobolev compact embeddings, we get that eventually extracting a subsequence via a diagonalization procedure, $m_{n} \rightarrow m_{k}$ weakly in $W^{1, r}\left(\mathbb{R}^{N}\right)$ for all $r<q$ and strongly in $L^{s}(K)$ for all $1 \leq s<q^{*}$ and for every compact $K \subset \mathbb{R}^{N}$, and $w_{n} \rightarrow w_{k}$ weakly in $L^{\frac{\gamma^{\prime} \alpha+\gamma^{\prime}}{\gamma^{\prime}+\alpha}}\left(\mathbb{R}^{N}\right)$. By using the fact that $\int_{\mathbb{R}^{N}} V(x) m_{n}(x) d x \leq C_{\varepsilon}$ and $(1.5)$, we get that we get that for all $R>1$,

$$
C_{\varepsilon} \geq \int_{\mathbb{R}^{N}} m_{n}(x) V(x) d x \geq \int_{|x|>R} m_{n}(x) V(x) d x \geq C R^{b} \int_{|x|>R} m_{n}(x) d x .
$$

So for every $\varepsilon>0$ fixed and all $\eta>0$, there exists $R>0$ for which $\int_{|x|>R} m_{n}(x) d x \leq \eta$ : up to extracting a subsequence we get that $m_{n} \rightarrow m_{k}$ in $L^{1}\left(\mathbb{R}^{N}\right)$, and so $\int_{\mathbb{R}^{N}} m_{k}(x) d x=M$. By boundedness of $m_{n}$ in $L^{s}\left(\mathbb{R}^{N}\right)$ for all $1 \leq s<q^{*}$, we then have $m_{n} \rightarrow m_{k}$ strongly in $L^{\alpha+1}\left(\mathbb{R}^{N}\right)$. 
Finally, observe that from (3.13), using (1.5), we conclude that $m_{k}\left(1+|x|^{b}\right) \in L^{1}\left(\mathbb{R}^{N}\right)$. Moreover, we get that

$$
\int_{\mathbb{R}^{N}}\left|w_{k}\right| d x \leq \int_{\mathbb{R}^{N}}\left|w_{k}\right|(1+|x|)^{b / \gamma} d x \leq\left(\int_{\mathbb{R}^{N}} \frac{\left|w_{k}\right|^{\gamma^{\prime}}}{m_{k}^{\gamma^{\prime}-1}} d x\right)^{1 / \gamma^{\prime}}\left(\int_{\mathbb{R}^{N}} m_{k}(1+|x|)^{b} d x\right)^{1 / \gamma},
$$

and so $w_{k}(1+|x|)^{b / \gamma} \in L^{1}\left(\mathbb{R}^{N}\right)$.

Therefore the convergence is sufficiently strong to assure that $\left(m_{k}, w_{k}\right) \in \mathcal{K}_{\varepsilon, M}$. We conclude that $\left(m_{k}, w_{k}\right)$ is a minimum of the energy, by the lower semicontinuity with respect to weak convergence of the functional $\int_{\mathbb{R}^{N}} m L\left(-\frac{w}{m}\right)+V(x) m d x$ and by using the fact that $F_{k}\left[m_{n}\right] \rightarrow$ $F_{k}\left[m_{k}\right]$, since $m_{n} \rightarrow m_{k}$ strongly in $L^{\alpha+1}\left(\mathbb{R}^{N}\right)$.

Using the minimizers we constructed in Proposition 3.3, we prove existence of a classical solution to (3.1).

Proposition 3.4. There exists a classical solution $\left(u_{k}, m_{k}, \lambda_{k}\right)$ to (3.1) that satisfies for some constant $C_{k, \varepsilon}>0$ the following inequalities

$$
\left|\nabla u_{k}(x)\right| \leq C_{k, \varepsilon}\left(1+|x|^{\frac{b}{\gamma}}\right) \quad u_{k}(x) \geq C_{k, \varepsilon}^{-1}\left(1+|x|^{1+\frac{b}{\gamma}}\right)-C_{k, \varepsilon} .
$$

Finally there exist $C, K>0$ not depending on $\varepsilon, k$ such that

$$
-K-C \varepsilon^{-\frac{\gamma^{\prime} \alpha N}{\gamma^{\prime}-\alpha N}} \leq \lambda_{k} \leq C \varepsilon^{-\frac{\gamma^{\prime} \alpha N}{\gamma^{\prime}-\alpha N}}+K .
$$

Proof. Let $\left(m_{k}, w_{k}\right)$ be a minimizer of $\mathcal{E}_{k}$. Define the space of test functions

$$
\mathcal{A}=\mathcal{A}_{b, \gamma}:=\left\{\psi \in C^{2}\left(\mathbb{R}^{N}\right): \limsup _{|x| \rightarrow \infty} \frac{|\nabla \psi(x)|}{|x|^{b / \gamma}}<\infty, \limsup _{|x| \rightarrow \infty} \frac{|\Delta \psi(x)|}{|x|^{b}}<\infty\right\} .
$$

Note that we also have, for all $\psi \in \mathcal{A}$,

$$
\limsup _{|x| \rightarrow \infty} \frac{|\psi(x)|}{|x|^{b / \gamma+1}}<\infty
$$

We claim that

$$
-\varepsilon \int_{\mathbb{R}^{N}} m_{k} \Delta \psi d x=\int_{\mathbb{R}^{N}} w_{k} \nabla \psi d x \quad \forall \psi \in \mathcal{A} .
$$

Indeed, consider a radial smooth cutoff function $\chi(x)$ which is identically equal to one in $B_{1}(0)$ and identically zero in $\mathbb{R}^{N} \backslash B_{2}(0)$. Set $\chi_{R}(x):=\chi(x / R)$; we have $\left|\nabla \chi_{R}\right| \leq C R^{-1}$ and $\left|\Delta \chi_{R}\right| \leq C R^{-2}$ on $\mathbb{R}^{N}$ for some positive constant $C$.

Since the equality $\varepsilon \Delta m_{k}=\operatorname{div} w_{k}$ holds in the weak sense on $\mathbb{R}^{N}$, we may multiply it by $\chi_{R} \psi$ with $\psi \in \mathcal{A}$ and integrate by parts to obtain

$$
-\varepsilon \int_{B_{2 R}} m_{k}\left(\chi_{R} \Delta \psi+2 \nabla \psi \cdot \nabla \chi_{R}+\psi \Delta \chi_{R}\right) d x=\int_{B_{2 R}} w_{k} \cdot\left(\chi_{R} \nabla \psi+\psi \nabla \chi_{R}\right) \cdot d x
$$

Note that for some positive $C$,

$$
\int_{\mathbb{R}^{N}}\left|w_{k} \nabla \psi\right| d x \leq C \int_{\mathbb{R}^{N}}\left|w_{k}\right|(1+|x|)^{b / \gamma} d x<\infty, \quad \int_{\mathbb{R}^{N}} m_{k}|\Delta \psi| d x \leq C \int_{\mathbb{R}^{N}} m_{k}(1+|x|)^{b} d x<\infty
$$

by the integrability properties (3.12). Moreover,

$$
\begin{aligned}
\int_{R \leq|x| \leq 2 R} m_{k}|\psi|\left|\Delta \chi_{R}\right| d x \leq C \int_{R \leq|x| \leq 2 R} & m_{k} \frac{(1+|x|)^{b / \gamma+1}}{R^{2}} d x \\
\leq C_{1} \int_{R \leq|x| \leq 2 R} m_{k}(1+|x|)^{b / \gamma-1} d x \rightarrow 0 \quad \text { as } R \rightarrow \infty &
\end{aligned}
$$


because $b / \gamma-1 \leq b$. Reasoning in a similar way, we also have that $\int_{R \leq|x| \leq 2 R} m_{k} \nabla \psi \cdot \nabla \chi_{R}$ and $\int_{R \leq|x| \leq 2 R} w_{k} \cdot \psi \nabla \chi_{R}$ converge to zero as $R \rightarrow \infty$. Equality (3.18) then follows by passing to the limit in (3.19).

Therefore, recalling the integrability properties of $m_{k}, w_{k}$ obtained in Proposition 3.3 , the problem of minimizing $\mathcal{E}_{k}$ on $\mathcal{K}_{\varepsilon, M}$ is equivalent to minimize $\mathcal{E}_{k}$ on $\mathcal{K}$, where

$\mathcal{K}:=\left\{(w, m) \in\left(L^{1} \cap W^{1, r}\right)\left(\mathbb{R}^{N}\right) \times L^{\frac{\gamma^{\prime}(\alpha+1)}{\gamma^{\prime}+\alpha}}\left(\mathbb{R}^{N}\right):(w, m)\right.$ satisfies (3.12), (3.18) $\left., m \geq 0, \int_{\mathbb{R}^{N}} m=M\right\}$

for some $r<q$. As in [8, Proposition 3.1], convexity of $L$ implies that $\left(m_{k}, w_{k}\right)$ is also a minimizer of the following convex functional on $\mathcal{K}$ :

$$
\widetilde{J}(m, w)=\int_{\mathbb{R}^{N}} m L\left(-\frac{w}{m}\right)+\left(V(x)+f_{k}\left[m_{k}\right]\right) m d x .
$$

We now aim to prove that

$$
\sup \left\{\lambda M:-\varepsilon \Delta \psi+H(\nabla \psi)+\lambda \leq V(x)+f_{k}\left[m_{k}\right] \text { on } \mathbb{R}^{N} \text { for some } \psi \in \mathcal{A}\right\}=\min _{(w, m) \in \mathcal{K}} \widetilde{J}(m, w) .
$$

We proceed as in [9, Theorem 3.5]: setting

$$
\mathcal{L}(m, w, \lambda, \psi):=\widetilde{J}(m, w)+\int_{\mathbb{R}^{N}} \varepsilon m \Delta \psi+w \nabla \psi-\lambda m d x+\lambda M,
$$

we have

$$
\min _{(m, w) \in \mathcal{K}} \widetilde{J}(m, w)=\min _{(m, w)} \sup _{(\lambda, \psi) \in \mathbb{R} \times \mathcal{A}} \mathcal{L}(m, w, \lambda, \psi),
$$

where the minimum in the right hand side has to be intended among couples $(m, w) \in\left(L^{1} \cap\right.$ $\left.W^{1, r}\right)\left(\mathbb{R}^{N}\right) \times L^{\frac{\gamma^{\prime}(\alpha+1)}{\gamma^{\prime}+\alpha}}\left(\mathbb{R}^{N}\right)$ for some $r<q$, satisfying (3.12). Note that $\mathcal{L}(\cdot, \cdot, \lambda, \psi)$ is convex, and $\mathcal{L}(m, w, \cdot, \cdot)$ is linear. Moreover, since $\mathcal{L}(\cdot, \cdot, \lambda, \psi)$ is weak-* lower semi-continuous, we can use the min-max theorem (see [6], Theorem 2.3.7]), to get

$$
\begin{aligned}
& \min _{(m, w)} \sup _{(\lambda, \psi) \in \mathbb{R} \times \mathcal{A}} \mathcal{L}(m, w, \lambda, \psi)=\sup _{(\lambda, \psi) \in \mathbb{R} \times \mathcal{A}} \min _{(m, w)} \mathcal{L}(m, w, \lambda, \psi)= \\
& \sup _{(\lambda, \psi) \in \mathbb{R} \times \mathcal{A}(m, w)} \min _{\mathbb{R}^{N}} m L\left(-\frac{w}{m}\right)+\left(V(x)+f_{k}\left[m_{k}\right]\right) m+\varepsilon m \Delta \psi+w \nabla \psi-\lambda m d x+\lambda M= \\
& \sup _{(\lambda, \psi) \in \mathbb{R} \times \mathcal{A}} \int_{\mathbb{R}^{N}} \min _{(m, w) \in \mathbb{R}_{\mathbb{R}^{N}}} m L\left(-\frac{w}{m}\right)+\left(V(x)+f_{k}\left[m_{k}\right]\right) m+\varepsilon m \Delta \psi+w \nabla \psi-\lambda m d x+\lambda M,
\end{aligned}
$$

where the interchange of the min and the integration is possible by standard results in convex optimisation. By computation, $\min _{(m, w) \in \mathbb{R} \times \mathbb{R}^{N}} m L\left(-\frac{w}{m}\right)+\left(V(x)+f_{k}\left[m_{k}\right]\right) m+\varepsilon m \Delta \psi+w \nabla \psi-\lambda m$ is zero whenever $\varepsilon \Delta \psi-H(\nabla \psi)-\lambda+\left(V(x)+f_{k}\left[m_{k}\right]\right)$ is positive, and it is $-\infty$ otherwise. Therefore, we have proven (3.20).

By Theorem 2.7, i), ii), there exists $u_{k} \in C^{2}\left(\mathbb{R}^{N}\right)$ such that

$$
-\varepsilon \Delta u_{k}+H\left(\nabla u_{k}\right)+\lambda_{k}=V(x)+f_{k}\left[m_{k}\right] \quad \text { on } \mathbb{R}^{N},
$$

and which satisfies

$$
\left|\nabla u_{k}(x)\right| \leq C_{k, \varepsilon}(1+|x|)^{\frac{b}{\gamma}} \quad u_{k}(x) \geq C_{k, \varepsilon}|x|^{\frac{b}{\gamma}+1}-C_{k, \varepsilon}^{-1}
$$

for some $C_{k, \varepsilon}>0$.

Moreover,

$$
\varepsilon\left|\Delta u_{k}(x)\right| \leq\left|H\left(\nabla u_{k}(x)\right)\right|+\left|\lambda_{k}\right|+V(x)-f_{k}\left[m_{k}\right] \leq C_{k, \varepsilon}(1+|x|)^{b} \quad \text { on } \mathbb{R}^{N}
$$


so $u_{k} \in \mathcal{A}$. Thus, the supremum in the left hand side of (3.20) is achieved by $\lambda_{k}$, and it holds true that

$$
\lambda_{k} M=\widetilde{J}\left(m_{k}, w_{k}\right)=\mathcal{E}_{k}\left(m_{k}, w_{k}\right)+\int_{\mathbb{R}^{N}} f_{k}\left[m_{k}\right] m_{k} d x-F\left[m_{k}\right] .
$$

This gives in particular (3.16), using Lemma 3.1, estimates (3.10) and recalling Proposition 3.2 and assumptions (1.3), (3.2) and (3.4).

We now use (3.22), (3.21) and (3.18) with $\psi=u_{k}$ to get

$$
\begin{aligned}
0=\int_{\mathbb{R}^{N}}\left(L\left(-\frac{w_{k}}{m_{k}}\right)+V(x)-m_{k}^{\alpha}-\lambda_{k}\right) & m_{k} d x=\int_{\mathbb{R}^{N}}\left(L\left(-\frac{w_{k}}{m_{k}}\right)-\varepsilon \Delta u_{k}+H\left(\nabla u_{k}\right)\right) m_{k} d x \\
= & \int_{\mathbb{R}^{N}}\left(L\left(-\frac{w_{k}}{m_{k}}\right)+H\left(\nabla u_{k}\right)+\nabla u_{k} \cdot \frac{w_{k}}{m_{k}}\right) m_{k} d x,
\end{aligned}
$$

that implies

$$
\frac{w_{k}}{m_{k}}=-\nabla H\left(\nabla u_{k}\right) \quad \text { on the set }\left\{m_{k}>0\right\} .
$$

Hence, the Kolmogorov equation $\varepsilon \Delta m_{k}+\operatorname{div}\left(m_{k} \nabla H\left(\nabla u_{k}\right)\right)=0$ holds in the weak sense, and by elliptic regularity we conclude that $\left(u_{k}, m_{k}, \lambda_{k}\right)$ is a classical solution to (1.1).

Remark 3.5. Note that if we assume that the local term $f$ satisfies (1.9) instead of (1.3), then the same argument as above applies. In particular there exists a classical solution $\left(u_{k}, m_{k}, \lambda_{k}\right)$ to (3.1) such that

$$
\begin{gathered}
\left|\nabla u_{k}(x)\right| \leq C_{k, \varepsilon}\left(1+|x|^{\frac{b}{\gamma}}\right) \quad u_{k}(x) \geq C_{k, \varepsilon}^{-1}\left(1+|x|^{1+\frac{b}{\gamma}}\right)-C_{k, \varepsilon}, \\
\int_{\mathbb{R}^{N}} m_{k}^{\alpha+1} d x, \int_{\mathbb{R}^{N}} m_{k}(x) V(x) d x \leq C \varepsilon^{-\frac{\gamma^{\prime} \alpha N}{\gamma^{\prime}-\alpha N}}+K .
\end{gathered}
$$

We finally prove that every $m_{k}$ is bounded from above in $\mathbb{R}^{N}$ (this is not obvious from Proposition 3.4 unless $\gamma^{\prime}>N$ ). Note that the following result does not provide uniform bounds with respect to $k$. These will be produced in Theorem 4.1 using a much more involved argument.

Proposition 3.6. Let $\left(u_{k}, m_{k}, \lambda_{k}\right)$ be as in Proposition 3.4. Then, $m_{k}$ is bounded in $L^{\infty}\left(\mathbb{R}^{N}\right)$.

Proof. Let $\phi(x)=u_{k}(x)^{p}$, for $p>1$ to be chosen later. Using the fact that $u_{k}$ is a classical solution to the HJB equation, we get

$$
\begin{aligned}
-\varepsilon \Delta \phi+ & \nabla H\left(\nabla u_{k}\right) \cdot \nabla \phi=p u_{k}^{p-1}\left(-\Delta u_{k}-(p-1) \frac{\left|\nabla u_{k}\right|^{2}}{u_{k}}+\nabla H\left(\nabla u_{k}\right) \cdot \nabla u_{k}\right) \\
& =p u_{k}^{p-1}\left(-\Delta u_{k}+H\left(\nabla u_{k}\right)-(p-1) \frac{\left|\nabla u_{k}\right|^{2}}{u_{k}}-H\left(\nabla u_{k}\right)+\nabla H\left(\nabla u_{k}\right) \cdot \nabla u_{k}\right) \\
& =p u_{k}^{p-1}\left(-(p-1) \frac{\left|\nabla u_{k}\right|^{2}}{u_{k}}-H\left(\nabla u_{k}\right)+\nabla H\left(\nabla u_{k}\right) \cdot \nabla u_{k}-\lambda+f_{k}\left[m_{k}\right]+V\right) .
\end{aligned}
$$

Observe that by (1.2), (1.5), (3.15) and the fact that $f_{k}\left[m_{k}\right]$ is bounded on $\mathbb{R}^{N}$, there exist large $R$ and $C$ such that

$$
\begin{aligned}
G(x) \geq K^{-1}\left|\nabla u_{k}\right|^{\gamma}-(p-1) & \frac{\left|\nabla u_{k}\right|^{2}}{u_{k}}-K-\lambda+f_{k}\left[m_{k}\right]+V(x) \\
& \geq(p-1)\left|\nabla u_{k}\right|^{\gamma}\left(\frac{1}{K(p-1)}-\frac{\left|\nabla u_{k}\right|^{2-\gamma}}{u_{k}}\right)-C+C_{V}^{-1}|x|^{b} \geq 1 \quad \text { for all }|x|>R .
\end{aligned}
$$

Hence, again by (3.15), for all $|x|>R$

$$
-\varepsilon \Delta \phi+\nabla H\left(\nabla u_{k}\right) \cdot \nabla \phi \geq c|x|^{(1+b / \gamma)(p-1)} .
$$

In view of [28, Proposition 2.6], we have $|x|^{(1+b / \gamma)(p-1)} m_{k} \in L^{1}\left(\mathbb{R}^{N}\right)$. Recall now that $\left|\nabla H\left(\nabla u_{k}\right)\right| \leq$ $C(1+|x|)^{\frac{b}{\gamma^{\prime}}}$ by (3.15). Therefore, by choosing $p$ large enough, $\left|\nabla H\left(\nabla u_{k}\right)\right|^{s} m_{k} \in L^{1}\left(\mathbb{R}^{N}\right)$ for some $s>N$. We conclude boundedness of $m_{k}$ in $L^{\infty}$ by [28, Theorem 3.5]. 


\section{Existence of a solution to the MFG system for $\varepsilon>0$}

Our aim is to pass to the limit $k \rightarrow \infty$ for solutions to (3.1).

\subsection{A priori $L^{\infty}$ bounds}

We need first a priori $L^{\infty}$ bounds on $m_{k}$ that are independent w.r.t. $k$. These will be achieved by a blow-up argument, as proposed in [12] for systems set on the flat torus $\mathbb{T}^{N}$. Here, the unbounded space $\mathbb{R}^{N}$ and the presence of the unbounded term $V$ make the argument much more involved than the one in 12 . To control the points $x_{k} \in \mathbb{R}^{N}$ where $m_{k}\left(x_{k}\right)$ possibly explodes, some delicate estimates on the decay (in $L^{1}$ ) of its renormalization will be produced.

We provide a more general result, that will be used also in the rescaled framework (Section 51). Let $r_{k}, s_{k}, t_{k}$ be bounded sequences of positive real numbers.

Theorem 4.1. Let $\left(u_{k}, \lambda_{k}, m_{k}\right)$ be a classical solution to the mean field game system

$$
\left\{\begin{array}{l}
-\Delta u+r_{k}^{\gamma} H\left(r_{k}^{-1} \nabla u\right)+\lambda_{k}=g_{k}[m]+s_{k} V\left(t_{k} x\right), \\
-\Delta m-\operatorname{div}\left(m r_{k}^{\gamma-1} \nabla H\left(r_{k}^{-1} \nabla u\right)\right)=0 \\
\int_{\mathbb{R}^{N}} m d x=M
\end{array}\right.
$$

where $g_{k}: L^{1}\left(\mathbb{R}^{N}\right) \rightarrow L^{1}\left(\mathbb{R}^{N}\right)$ are so that for all $m \in L^{\infty}\left(\mathbb{R}^{N}\right) \cap L^{1}\left(\mathbb{R}^{N}\right)$ and for all $k$,

$$
\left\|g_{k}[m]\right\|_{L^{\infty}\left(\mathbb{R}^{N}\right)} \leq K\left(\|m\|_{L^{\infty}\left(\mathbb{R}^{N}\right)}^{\alpha}+1\right)
$$

for some $K>0$. Suppose also that for all $k, u_{k}$ is bounded from below and $m_{k}$ is bounded from above on $\mathbb{R}^{N}$. Then, there exists a constant $C$ independent of $k$ such that

$$
\left\|m_{k}\right\|_{L^{\infty}} \leq C
$$

Proof. We argue by contradiction, so we assume that

$$
\sup _{\mathbb{R}^{N}} m_{k}=L_{k} \rightarrow+\infty
$$

We divide the proof in several steps.

\section{Step 1: rescaling of the solutions}

Let

$$
\mu_{k}:=L_{k}^{-\beta} \quad \beta=\alpha \frac{\gamma-1}{\gamma}>0 .
$$

So, observe that $\mu_{k} \rightarrow 0$ as $k \rightarrow 0$. Since $u_{k}$ is bounded by below, up to adding a suitable constant we can assume that $\min _{\mathbb{R}^{N}} u_{k}=0$. We define the following rescaling

$$
\left\{\begin{array}{l}
v_{k}(x)=\mu_{k}^{\frac{2-\gamma}{\gamma-1}} u_{k}\left(\mu_{k} x\right)+1 \\
n_{k}(x)=L_{k}^{-1} m_{k}\left(\mu_{k} x\right) .
\end{array}\right.
$$

Note that $0 \leq n_{k}(x) \leq 1$. Moreover, due to (1.4),

$$
\int_{\mathbb{R}^{N}} n_{k}(x) d x=M L_{k}^{\frac{\alpha N(\gamma-1)}{\gamma}-1} \rightarrow 0
$$

and $\min v_{k}=1$. We define

$$
H_{k}(q)=\mu_{k}^{\frac{\gamma}{\gamma-1}} r_{k}^{\gamma} H\left(r_{k}^{-1} \mu_{k}^{\frac{1}{1-\gamma}} q\right), \quad \text { so } \quad \nabla H_{k}(q)=\mu_{k} r_{k}^{\gamma-1} \nabla H\left(r_{k}^{-1} \mu_{k}^{\frac{1}{1-\gamma}} q\right) \text {. }
$$

Recalling (1.2) we have that for all $q \in \mathbb{R}^{N}$,

$$
\begin{aligned}
& C_{H}|q|^{\gamma}-K \leq H_{k}(q) \leq C_{H}\left(|q|^{\gamma}+1\right), \\
& \left.\mid \nabla H_{k}(q)\right)\left.\left|\leq C_{H}\right| q\right|^{\gamma-1} \\
& \nabla H_{k}(q) \cdot q-H_{k}(q) \geq K^{-1}|q|^{\gamma}-K .
\end{aligned}
$$


Moreover, we define

$$
\tilde{g}_{k}(x)=\mu_{k}^{\frac{\gamma}{\gamma-1}} g_{k}\left[m_{k}\right]\left(\mu_{k} x\right) .
$$

Recalling that $0 \leq m_{k} \leq L_{k}$, by (4.1) we get that for all $x$ and for all $k$,

$$
\left|\tilde{g}_{k}(x)\right| \leq \mu_{k}^{\frac{\gamma}{\gamma-1}} K\left(L_{k}^{\alpha}+1\right) \leq 2 K
$$

where we used the fact that $\mu_{k}=L_{k}^{-\beta}$ with $\beta=\alpha \frac{\gamma-1}{\gamma}$. Finally, we let

$$
\tilde{\lambda}_{k}=\mu_{k}^{\frac{\gamma}{\gamma-1}} \lambda_{k}=\frac{1}{L_{k}^{\alpha}} \lambda_{k}
$$

and we observe that

$$
\left|\tilde{\lambda}_{k}\right| \leq C
$$

Finally, let

$$
V_{k}(x)=\mu_{k}^{\frac{\gamma}{\gamma-1}} s_{k} V\left(\mu_{k} t_{k} x\right)
$$

By assumption (1.5), we get

$$
s_{k} \mu_{k}^{\frac{\gamma}{\gamma-1}} C_{V}^{-1}\left(\max \left\{\left|t_{k} \mu_{k} x\right|-C_{V}, 0\right\}\right)^{b} \leq V_{k}(x) \leq C_{V}\left(1+\sigma_{k}|x|^{b}\right),
$$

where

$$
\sigma_{k}:=\mu_{k}^{\frac{\gamma}{\gamma-1}+b} s_{k} t_{k}^{b} \rightarrow 0 \quad \text { as } k \rightarrow \infty .
$$

In particular we also have the following bound from below for $V_{k}$,

$$
V_{k}(x) \geq \frac{C_{V}^{-1}}{2^{b}} \sigma_{k}|x|^{b} \quad \text { for all }|x| \geq 2 C_{V}\left(t_{k} \mu_{k}\right)^{-1} .
$$

An easy computation shows that by rescaling we have that $\left(v_{k}, n_{k}, \tilde{\lambda}_{k}\right)$ is a solution to

$$
\left\{\begin{array}{l}
-\Delta v_{k}+H_{k}\left(\nabla v_{k}\right)+\tilde{\lambda}_{k}=\tilde{g}_{k}(x)+V_{k}(x), \\
-\Delta n_{k}-\operatorname{div}\left(n_{k} \nabla H_{k}\left(\nabla v_{k}\right)\right)=0 .
\end{array}\right.
$$

Step 2: a priori bounds on the rescaled solution to the Hamilton-Jacobi equation. We observe that by Theorem 2.5 and (4.6), there exists $C>0$, independent of $k$, such that

$$
\left|\nabla v_{k}(x)\right| \leq C\left(1+\sigma_{k}^{\frac{1}{\gamma}}|x|^{\frac{b}{\gamma}}\right) \quad \text { on } \mathbb{R}^{N} .
$$

We recall that we assumed $v_{k}\left(\hat{x}_{k}\right)=\min v_{k}=1$. Since $v_{k}$ is a classical solution to (4.8), at a minimum point $\hat{x}_{k}$ we have, by (4.3), (4.4), (4.5) and (4.7),

$$
\sigma_{k}\left|\hat{x}_{k}\right|^{b} \leq C
$$

Therefore, by using this estimate and (4.9), since $\left|v_{k}(0)\right| \leq\left|v_{k}\left(\hat{x}_{k}\right)\right|+\left|\hat{x}_{k}\right| \sup _{|y| \leq\left|\hat{x}_{k}\right|}\left|\nabla u_{k}(y)\right|$ we obtain

$$
\left|v_{k}(0)\right| \leq 1+C\left(1+\sigma_{k}^{\frac{1}{\gamma}}\left|\hat{x}_{k}\right|^{1+\frac{b}{\gamma}}\right) \leq C_{1}\left(1+\sigma_{k}^{-\frac{1}{b}}\right)
$$

and then again by (4.9),

$$
\left|v_{k}(x)\right| \leq C\left(1+\sigma_{k}^{-\frac{1}{b}}+\sigma_{k}^{\frac{1}{\gamma}}|x|^{\frac{b}{\gamma}+1}\right) \quad \text { on } \mathbb{R}^{N} .
$$

Let $\chi$ be a smooth function $\chi:[0,+\infty) \rightarrow[0,+\infty)$ such that $\chi \equiv 0$ in $(0,1 / 2) \cup(3 / 2,+\infty)$, $\chi(1)>0$ and that $\left|\chi^{\prime}\right|,\left|\chi^{\prime \prime}\right| \leq 1$. We fix $\tilde{x} \in \mathbb{R}^{N}$ such that $|\tilde{x}|>4 C_{V}\left(t_{k} \mu_{k}\right)^{-1}$, and we denote by

$$
w(x)=K \sigma_{k}^{\frac{1}{\gamma}}|\tilde{x}|^{1+\frac{b}{\gamma}} \chi\left(\frac{|x|}{|\tilde{x}|}\right)
$$


where $K \geq 0$ has to be chosen. We have that $w(x) \leq v_{k}(x)$ for all $x$ such that $|x| \geq \frac{3}{2}|\tilde{x}|$ or $|x| \leq \frac{1}{2}|\tilde{x}|$. Moreover, for $x$ such that $\frac{1}{2}|\tilde{x}| \leq|x| \leq \frac{3}{2}|\tilde{x}|$ we have $|x|>2 C_{V}\left(\mu_{k} t_{k}\right)^{-1}$, so using the estimates (4.3), (4.4), (4.5) and (4.7),

$$
-\Delta w+H_{k}(\nabla w)+\tilde{\lambda}_{k}-\tilde{g}_{k}(x)-V_{k}(x) \leq K N \sigma_{k}^{\frac{1}{\gamma}}|\tilde{x}|^{\frac{b}{\gamma}-1}+C_{H} K^{\gamma} \sigma_{k}|\tilde{x}|^{b}+C-\frac{C_{V}^{-1}}{2^{b}} \sigma_{k}|\tilde{x}|^{b} .
$$

Note that there exist $K>0$ small and $C_{2}>0$ large, depending only $C_{V}$ and $C_{H}$ and not on $|\tilde{x}|$, $k$, such that the right-hand side of the last expression is negative if

$$
\sigma_{k}|\tilde{x}|^{b} \geq C_{2}
$$

(this also implies that $t_{k} \mu_{k}|\tilde{x}|>4 C_{V}$, as required). The test function $w$ is then a subsolution of the HJB equation in (4.8), therefore by comparison we get that,

$$
v_{k}(\tilde{x}) \geq K \chi(1) \sigma_{k}^{\frac{1}{\gamma}}|\tilde{x}|^{1+\frac{b}{\gamma}} .
$$

By arbitrariness of $\tilde{x}$ we conclude that, for some $C>0$,

$$
v_{k}(x) \geq C \sigma_{k}^{\frac{1}{\gamma}}|x|^{\frac{b}{\gamma}+1} \quad \text { for all } \sigma_{k}|x|^{b} \geq C_{2} .
$$

Step 3: estimates on the (approximate) maxima of $n_{k}$.

We now fix $0<\delta<<1$ and $x_{k}$ such that $n_{k}\left(x_{k}\right)=1-\delta$. Two possibilities may arise: either $\lim _{k} \sigma_{k}\left|x_{k}\right|^{b}=+\infty$ up to some subsequence, or there exists $C>0$ such that $\sigma_{k}\left|x_{k}\right|^{b} \leq C$. We rule out the second possibility by contradiction. Suppose indeed that there exists $C>0$ such that $\sigma_{k}\left|x_{k}\right|^{b} \leq C$. By (4.9), $\left|\nabla v_{k}\right| \leq C$ on $B_{2}\left(x_{k}\right)$ for some $C>0$. Therefore, using the fact that $n_{k}$ solves the second equation in (4.8), the elliptic estimates in Proposition 2.4, (4.3), the interpolation inequality $\|n\|_{q} \leq\|n\|_{1}^{1 / q}\|n\|_{\infty}^{1-1 / q}$ and the fact that $0 \leq n_{k} \leq 1$, we get for all $q>1$,

$$
\left\|n_{k}\right\|_{W^{1, q}\left(B_{1}\left(x_{k}\right)\right)} \leq C\left(1+\left\|\nabla H_{k}\left(\nabla v_{k}\right)\right\|_{L^{\infty}\left(B_{2}\left(x_{k}\right)\right)}\right)\left\|n_{k}\right\|_{L^{1}\left(B_{2}\left(x_{k}\right)\right)}^{1 / q} \leq C_{q}
$$

for some $C_{q}>0$ depending on $q$. This implies, choosing $q>N$, that for all $\theta \in(0,1)$ there exists $C_{\theta}$ depending on $\theta$ (but not on $k$ ) such that $\left\|n_{k}\right\|_{C^{0, \theta}\left(B_{1}\left(x_{k}\right)\right)} \leq C_{\theta}$. Recalling that $n_{k}\left(x_{k}\right)=1-\delta$, we can fix $r<1$ such that $n_{k}(x) \geq \frac{1}{2}$ for all $x \in B_{r}\left(x_{k}\right)$. It is sufficient to choose $r=C_{\theta}^{-1 / \theta}(1 / 2-\delta)^{1 / \theta}$. Therefore we have, by (4.2),

$$
0<\frac{1}{2} \omega_{N} r^{N} \leq \int_{B_{r}\left(x_{k}\right)} n_{k}(x) d x \leq \int_{\mathbb{R}^{N}} n_{k}(x) d x=M L_{k}^{\frac{\alpha N(\gamma-1)}{\gamma}-1} \rightarrow 0 .
$$

This gives a contradiction. Then we deduce that, up to a subsequence,

$$
\lim _{k} \sigma_{k}\left|x_{k}\right|^{b}=+\infty
$$

\section{Step 4: construction of a Lyapunov function.}

Let $\phi(x)=v_{k}(x)^{p}$, for $p>1$ to be chosen later. Using the fact that $v_{k}$ is a classical solution to (4.8) (arguing as in (3.23)) we get

$$
\begin{aligned}
-\Delta \phi+\nabla H_{k}\left(\nabla v_{k}\right) \cdot \nabla \phi=p v_{k}^{p-1}\left(-\Delta v_{k}-(p-1) \frac{\left|\nabla v_{k}\right|^{2}}{v_{k}}+\nabla H_{k}\left(\nabla v_{k}\right) \cdot \nabla v_{k}\right) \\
=p v_{k}^{p-1}\left(-(p-1) \frac{\left|\nabla v_{k}\right|^{2}}{v_{k}}-H_{k}\left(\nabla v_{k}\right)+\nabla H_{k}\left(\nabla v_{k}\right) \cdot \nabla v_{k}-\tilde{\lambda}_{k}+\tilde{g}_{k}(x)+V_{k}(x)\right) .
\end{aligned}
$$

We denote by

$$
G_{k}(x)=-(p-1) \frac{\left|\nabla v_{k}\right|^{2}}{v_{k}}-H_{k}\left(\nabla v_{k}\right)+\nabla H_{k}\left(\nabla v_{k}\right) \cdot \nabla v_{k}-\tilde{\lambda}_{k}+\tilde{g}_{k}(x)+V_{k}(x) .
$$


Using the previous computation and the fact that $n_{k}$ is a solution to (4.8), we get, by integrating by parts, that

$$
0=\int_{\mathbb{R}^{N}} n_{k}(x)\left(-\Delta \phi(x)+\nabla H_{k}\left(\nabla v_{k}(x)\right) \cdot \nabla \phi(x)\right) d x=p \int_{\mathbb{R}^{N}} n_{k}(x) G_{k}(x) \phi^{\frac{p-1}{p}}(x) d x .
$$

Therefore from this, for every $\Lambda>0$ we get

$$
\int_{\left\{\phi(x) \geq \Lambda^{p}\right\}} n_{k}(x) G_{k}(x) \phi^{\frac{p-1}{p}}(x) d x=-\int_{\left\{\phi(x) \leq \Lambda^{p}\right\}} n_{k}(x) G_{k}(x) \phi^{\frac{p-1}{p}}(x) d x .
$$

Observe that by (4.3), (4.4), (4.5) and (4.7) we get that for all $t_{k} \mu_{k}|x| \geq 2 C_{V}$,

$$
\begin{aligned}
G_{k}(x) \geq K^{-1}\left|\nabla v_{k}\right|^{\gamma}-(p-1) & \frac{\left|\nabla v_{k}\right|^{2}}{v_{k}}-K-\tilde{\lambda}_{k}+\tilde{g}_{k}(x)+V_{k}(x) \\
& \geq(p-1)\left|\nabla v_{k}\right|^{\gamma}\left(\frac{1}{K(p-1)}-\frac{\left|\nabla v_{k}\right|^{2-\gamma}}{v_{k}}\right)-C+C_{V} \sigma_{k}|x|^{b} .
\end{aligned}
$$

We first claim that by (4.9) and (4.11), $\frac{1}{K(p-1)}-\frac{\left|\nabla v_{k}\right|^{2-\gamma}}{v_{k}}$ is positive if $\sigma_{k}|x|^{b} \geq C_{2}$, eventually enlarging $C_{2}$ in (4.11). Indeed,

$$
\frac{\left|\nabla v_{k}(x)\right|^{2-\gamma}}{v_{k}(x)} \leq C \frac{\left[1+\sigma_{k}^{\frac{1}{\gamma}}|x|^{\frac{b}{\gamma}}\right]^{2-\gamma}}{\left[\sigma_{k}^{\frac{1}{\gamma}}|x|^{\frac{b}{\gamma}}\right]|x|} \leq \frac{C_{H}}{p-1}
$$

whenever $\sigma_{k}|x|^{b}$ is large enough. This implies that for all $\sigma_{k}|x|^{b} \geq C_{2}$, by (4.16) we have $G_{k}(x) \geq$ $-C$. On the other hand, again by the gradient bounds in (4.9) we have that $\left|\nabla v_{k}(x)\right| \leq C\left(1+C_{2}\right)$ on the set $\sigma_{k}|x|^{b} \leq C_{2}$, so (4.16) and $\min v_{k}=1$ again guarantee that $G_{k}(x) \geq-C_{3}$. In conclusion, there exists $C>0$ such that

$$
G_{k}(x) \geq-C \quad \forall x \in \mathbb{R}^{N} .
$$

Therefore, going back to (4.15), recalling (4.2), we obtain that

$$
\begin{aligned}
\int_{\left\{\phi(x) \geq \Lambda^{p}\right\}} n_{k}(x) G_{k}(x)\left(\frac{\phi(x)}{\Lambda^{p}}\right)^{\frac{p-1}{p}} d x \leq C \int_{\left\{\phi(x) \leq \Lambda^{p}\right\}} n_{k}(x) d x \leq C \int_{\mathbb{R}^{N}} n_{k}(x) d x \\
=C M \mu_{k}^{-N+\frac{\gamma}{\alpha(\gamma-1)}} \rightarrow 0
\end{aligned}
$$

as $k \rightarrow \infty$.

Note that by (4.16) and (4.17), if $x$ is such that $G_{k}(x) \leq 0$, then necessarily $\sigma_{k}|x|^{b} \leq C$ for some $C>0$. Hence, by (4.10), we get that $v_{k}(x) \leq C_{3}\left(1+\sigma_{k}^{-\frac{1}{b}}\right)$. Therefore if we choose $\Lambda=\Lambda_{k}=K \sigma_{k}^{-\frac{1}{b}}$ for a sufficiently large $K>0$, we get that $G_{k}(x)>0$ in the set $\left\{x \mid \phi(x) \geq \Lambda^{p}\right\}$.

Step 5: integral estimates on $n_{k}$.

Arguing as in the end of Step 4, we may choose $K$ big enough so that $G_{k}(x) \geq 1$ in the set $\left\{x \mid \phi(x) \geq \Lambda^{p}\right\}$, where $\Lambda_{k}=K \sigma_{k}^{-\frac{1}{b}}$. If $k$ is sufficiently large, by (4.11) and (4.13) it follows that for some $C>0$,

$$
\begin{aligned}
& v_{k}(x) \geq C \sigma_{k}^{\frac{1}{\gamma}}\left|x_{k}\right|^{1+\frac{b}{\gamma}} \quad \text { in } B_{1}\left(x_{k}\right), \text { and } \\
& B_{1}\left(x_{k}\right) \subseteq\left\{x \mid \phi(x) \geq \Lambda^{p}\right\}
\end{aligned}
$$


Therefore, we may conclude that

$$
\begin{array}{r}
\int_{\left\{\phi(x) \geq \Lambda^{p}\right\}} n_{k}(x) G_{k}(x)\left(\frac{\phi(x)}{\Lambda^{p}}\right)^{\frac{p-1}{p}} d x \geq C\left(\frac{\sigma_{k}^{\frac{1}{\gamma}}\left|x_{k}\right|^{1+\frac{b}{\gamma}}}{\sigma_{k}^{-\frac{1}{b}}}\right)^{p-1} \int_{B_{1}\left(x_{k}\right)} n_{k}(x) d x \\
\geq C\left(\sigma_{k}^{\frac{1}{\gamma}}\left|x_{k}\right|^{\frac{b}{\gamma}}\right)^{p-1} \int_{B_{1}\left(x_{k}\right)} n_{k}(x) d x,
\end{array}
$$

that together with (4.18) gives

$$
\int_{B_{1}\left(x_{k}\right)} n_{k}(x) d x \leq\left(\sigma_{k}^{\frac{1}{\gamma}}\left|x_{k}\right|^{\frac{b}{\gamma}}\right)^{1-p}
$$

for all $k$ large.

Reasoning as in Step 3 (see in particular (4.12)), by Proposition 2.4, (4.3), (4.9) and (4.20), we get that for all $q>1$,

$$
\begin{aligned}
\left\|n_{k}\right\|_{W^{1, q}\left(B_{1 / 2}\left(x_{k}\right)\right)} \leq C\left(1+\left\|\nabla H_{k}\left(\nabla v_{k}\right)\right\|_{L^{\infty}\left(B_{1}\left(x_{k}\right)\right)}\right)\left\|n_{k}\right\|_{L^{1}\left(B_{1}\left(x_{k}\right)\right)}^{1 / q} & \\
& \leq C_{4}\left[1+\left(\sigma_{k}^{\frac{1}{\gamma}}\left|x_{k}\right|^{\frac{b}{\gamma}}\right)^{\gamma-1}\right]\left(\sigma_{k}^{\frac{1}{\gamma}}\left|x_{k}\right|^{\frac{b}{\gamma}}\right)^{(1-p) / q} \leq 1,
\end{aligned}
$$

whenever $p$ is such that $\gamma-1+(1-p) / q<0$ and $k$ is large (recall that we are supposing $\left.\sigma_{k}^{\frac{1}{\gamma}}\left|x_{k}\right|^{\frac{b}{\gamma}} \rightarrow+\infty\right)$

Therefore, we may conclude as in Step 3: choosing $q>N$, for some $\theta \in(0,1)$ there exists $C_{\theta}$ such that $\left\|n_{k}\right\|_{C^{0, \theta}\left(B_{1 / 2}\left(x_{k}\right)\right)} \leq C_{\theta}$. Since $n_{k}\left(x_{k}\right)=1-\delta$, we can fix $r<1$ such that $n_{k}(x) \geq \frac{1}{2}$ for all $x \in B_{r}\left(x_{k}\right)$. Finally, by (4.2)

$$
0<\frac{1}{2} \omega_{N} r^{N} \leq \int_{B_{r}\left(x_{k}\right)} n_{k}(x) d x \leq \int_{\mathbb{R}^{N}} n_{k}(x) d x=M L_{k}^{\frac{\alpha N(\gamma-1)}{\gamma-1}} \rightarrow 0 .
$$

That gives a contradiction and rules out the possibility that $\sigma_{k}\left|x_{k}\right|^{b} \rightarrow+\infty$. Therefore, $L_{k} \rightarrow+\infty$ is impossible.

\subsection{Existence of a solution to the MFG system}

Using the a priori bounds we obtained, we can pass to the limit in $k$ in the MFG system (3.1) to get a solution to (1.1) for every $\varepsilon>0$.

Proof of Theorem 1.1. First, by Proposition 3.4 the existence for all $k$ of a classical solution $\left(u_{k}, m_{k}, \lambda_{k}\right)$ to (3.1) follows. By (3.16), up to passing to a subsequence we have that $\lambda_{k} \rightarrow \lambda_{\varepsilon}$.

Note that by Propositions 3.4 and 3.6 $u_{k}$ and $m_{k}$ are bounded by below and above respectively, so due to Theorem 4.1 (with $g[m]=f_{k}[m]$ and $r_{k}=s_{k}=t_{k}=1$ for all $k$ ), we get that there exists $C_{\varepsilon}>0$ independent of $k$ (but eventually on $\varepsilon>0$ ) such that $\left\|m_{k}\right\|_{L^{\infty}\left(\mathbb{R}^{N}\right)} \leq C_{\varepsilon}$. Using Theorem 2.5] this implies that $\left|\nabla u_{k}(x)\right| \leq C_{\varepsilon}\left(1+|x|^{\frac{b}{\gamma}}\right)$, for some $C_{\varepsilon}$ independent of $k$. We can normalize $u_{k}(0)=0$ and using Ascoli-Arzelá theorem we can extract by a diagonalization procedure a sequence $u_{k}$ such that $u_{k} \rightarrow u_{\varepsilon}$ locally uniformly in $\mathbb{R}^{N}$. Moreover, by using the estimates and the equation we have that actually $u_{k} \rightarrow u_{\varepsilon}$ locally uniformly in $C^{1}$. Note that, denoting by $x_{k}$ a minimum point of $u_{k}$ on $\mathbb{R}^{N}$, we have by the HJB equation that

$$
H(0)+\lambda_{k}-f_{k}\left[m_{k}\right]\left(x_{k}\right) \geq V\left(x_{k}\right) .
$$

Coercivity (1.5) of $V$ and uniform boundedness of $\lambda_{k}$ and $f_{k}\left[m_{k}\right]$ guarantee that $x_{k}$ remains bounded, in particular that $u_{k} \geq-C$ on $\mathbb{R}^{N}$ by gradient bounds. Theorem 2.6 then applies, in particular $u_{k}(x) \geq C|x|^{1+\frac{b}{\gamma}}-C^{-1}$ for all $k$. This implies, passing to the limit, that

$$
u_{\varepsilon}(x) \geq C|x|^{1+\frac{b}{\gamma}}-C^{-1} \quad \text { on } \mathbb{R}^{N} .
$$


By the elliptic estimates in Proposition 2.4, we get that $m_{k} \rightarrow m_{\varepsilon}$ locally uniformly in $C^{0, \alpha}$ for all $\alpha \in(0,1)$ and weakly in $W^{1, p}\left(B_{R}\right)$ for every $p>1$ and $R>0$. Therefore we get that $u_{\varepsilon}$ is a solution in the viscosity sense of the Hamilton-Jacobi equation, by stability with respect to uniform convergence, and $m_{\varepsilon}$ is a weak solution to the Fokker-Planck equation, by strong convergence of $\nabla u_{k} \rightarrow \nabla u_{\varepsilon}$. Finally this implies, again by using the regularity of the HJB equation, that $u_{k} \rightarrow u_{\varepsilon}$ locally uniformly in $C^{2}$. Therefore, $u_{\varepsilon}, m_{\varepsilon}$ solve in classical sense the system (1.1).

Now we show that $\int_{\mathbb{R}^{N}} m_{\varepsilon}(x) d x=M$. We have that $m_{k} \rightarrow m_{\varepsilon}$ locally uniformly in $C^{0, \alpha}$ for every $\alpha \in(0,1)$. Moreover, due to (3.13) and to (1.5), we get that for all $R>1$,

$$
C_{\varepsilon} \geq \int_{\mathbb{R}^{N}} m_{k}(x) V(x) d x \geq \int_{|x|>R} m_{k}(x) V(x) d x \geq C R^{b} \int_{|x|>R} m_{k}(x) d x .
$$

This implies that $\int_{|x| \leq R} m_{k}(x) d x \geq M-C_{\varepsilon} R^{-b}$ and then by uniform convergence we get that for every $\varepsilon>0$, and $\eta>0$, there exists $R>0$ such that

$$
\int_{|x| \leq R} m_{\varepsilon}(x) d x \geq M-\eta .
$$

From this we can conclude that $m_{k} \rightarrow m_{\varepsilon}$ in $L^{1}\left(\mathbb{R}^{N}\right)$, that is $\int_{\mathbb{R}^{N}} m_{\varepsilon}(x) d x=M$. By boundedness of $m_{k}$ in $L^{\infty}$, it also follows that $m_{k} \rightarrow m_{\varepsilon}$ in $L^{\alpha+1}\left(\mathbb{R}^{N}\right)$.

Finally, we get that if $w_{\varepsilon}=-m_{\varepsilon} \nabla H\left(\nabla u_{\varepsilon}\right)$, then $\left(m_{\varepsilon}, w_{\varepsilon}\right) \in \mathcal{K}_{\varepsilon, M}$, due to the second equation in (1.1). Moreover, we have that if $m_{k} \rightarrow m$ strongly in $L^{\alpha+1}\left(\mathbb{R}^{N}\right)$, then, due to the Lebesgue dominated convergence theorem and (3.4), $F\left(m_{k} \star \chi_{k}\right) \rightarrow F(m)$ strongly in $L^{1}\left(\mathbb{R}^{N}\right)$. This implies that the energy $\mathcal{E}_{k} \Gamma$-converges to the energy $\mathcal{E}$, from which we conclude that $\left(m_{\varepsilon}, w_{\varepsilon}\right)$ is a minimizer of $\mathcal{E}$ in the set $\mathcal{K}_{\varepsilon, M}$.

Remark 4.2. Note that by the very same arguments, recalling Remark 3.5 , we have the existence of solutions also in the more general case that condition (1.9) is satisfied.

We conclude proving some estimates on the solution $\left(u_{\varepsilon}, m_{\varepsilon}, \lambda_{\varepsilon}\right)$ given in Theorem 1.1 that will be useful in the following.

Corollary 4.3. Let $\left(u_{\varepsilon}, m_{\varepsilon}, \lambda_{\varepsilon}\right)$ be as in Theorem 1.1. There exist constants $C, C_{1}, C_{2}, K, K_{1}, K_{2}>$ 0 independent of $\varepsilon$ such that

$$
\begin{gathered}
\int_{\mathbb{R}^{N}} m_{\varepsilon}\left|\nabla u_{\varepsilon}\right|^{\gamma} d x+\int_{\mathbb{R}^{N}} m_{\varepsilon}^{\alpha+1} d x+\int_{\mathbb{R}^{N}} m_{\varepsilon}(x) V(x) d x \leq C \varepsilon^{-\frac{\gamma^{\prime} \alpha N}{\gamma^{\prime}-\alpha N}}+K \\
-K_{1}-C_{1} \varepsilon^{-\frac{\gamma^{\prime} \alpha N}{\gamma^{\prime}-\alpha N}} \leq \lambda_{\varepsilon} \leq K_{2}-C_{2} \varepsilon^{-\frac{\gamma^{\prime} \alpha N}{\gamma^{\prime}-\alpha N}} .
\end{gathered}
$$

Proof. We observe that, by the arguments in the proof of Theorem 1.1 $m_{k} \rightarrow m_{\varepsilon}$ and $\left|\nabla u_{k}\right| \rightarrow$ $\left|\nabla u_{\varepsilon}\right|$ almost everywhere, and using the fact that $V(x) \geq 0$, we have that by Fatou lemma $\int_{\mathbb{R}^{N}} m_{\varepsilon}(x)\left|\nabla u_{\varepsilon}\right|^{\gamma} d x \leq \liminf _{k} \int_{\mathbb{R}^{N}} m_{k}(x)\left|\nabla u_{k}\right|^{\gamma} d x, \int_{\mathbb{R}^{N}} m_{\varepsilon}(x) V(x) d x \leq \liminf k_{k} \int_{\mathbb{R}^{N}} m_{k}(x) V(x) d x$ and $\int_{\mathbb{R}^{N}} m_{\varepsilon}^{\alpha+1} d x \leq \liminf _{k} \int_{\mathbb{R}^{N}} m_{k}^{\alpha+1} d x$. So inequality (3.13) gives immediately (4.22).

Now we prove (4.23). Note that the estimate from below is a direct consequence of (3.16). So, it remains to show that $\lambda_{\varepsilon} \leq C_{2}-C_{2} \varepsilon^{-\frac{\gamma^{\prime} \alpha N}{\gamma^{\prime}-\alpha N}}$. Recalling that formula (3.22) holds and $\int f(m) m-F(m) \leq 2 K M$ by (1.3), it is sufficient to show that

$$
\inf _{(m, w) \in \mathcal{K}_{\varepsilon, M}} \mathcal{E}(m, w) \leq-C_{2} \varepsilon^{-\frac{\gamma^{\prime} \alpha N}{\gamma^{\prime}-\alpha N}}+C_{2}
$$

where $C_{2}$ is a constant depending only on $N, M, C_{L}, \gamma, \alpha, V$. We construct a couple $(m, w) \in \mathcal{K}_{\varepsilon, M}$ as follows. First of all we consider a smooth function $\phi:[0,+\infty) \rightarrow \mathbb{R}$ which solves the following ordinary differential equation

$$
\left\{\begin{array}{l}
\phi^{\prime}(r)=-\phi(r)\left(1+\phi(r)^{\alpha}\right)^{\frac{1}{\gamma^{\prime}}} \\
\phi(0)=\frac{1}{2}
\end{array}\right.
$$


Then, it is easy to check that $0<\phi(r) \leq \frac{1}{2} e^{-r}$. We define $m(x)=A \phi(\tau|x|)$, where $A, \tau$ are constants to be fixed, and $w(x)=\varepsilon \nabla m(x)$.

First of all we impose

$$
M=\int_{\mathbb{R}^{N}} m(x) d x=\frac{A}{\tau^{N}} \int_{\mathbb{R}^{N}} \phi(|y|) d y=\frac{A}{\tau^{N}} C^{-1},
$$

recalling that $\phi$ is exponentially decreasing. So $A=M \tau^{N} C$, where $C^{-1}=\int_{\mathbb{R}^{N}} \phi(|y|) d y$.

Observe also that

$$
\int_{\mathbb{R}^{N}} m^{\alpha+1}(x) d x=M^{\alpha+1} \tau^{\alpha N} C^{\alpha+1} \int_{\mathbb{R}^{N}} \phi^{\alpha+1}(|y|) d y=M^{\alpha+1} \tau^{\alpha N} C^{\alpha+1} C_{\alpha}
$$

where $C_{\alpha}=\int_{\mathbb{R}^{N}} \phi^{\alpha+1}(|y|) d y$.

We check, recalling that the growth condition (1.5), that the following holds

$$
\int_{\mathbb{R}^{N}} m(x) V(x) d x=M C \int_{\mathbb{R}^{N}} V\left(\frac{y}{\tau}\right) \phi(|y|) d y=C_{1} \frac{1}{\tau^{b}},
$$

where $K$ is a constant depending on $N, \phi, C_{0}$.

Moreover, we compute, recalling that $\phi$ solves the ODE

$$
|w|^{\gamma^{\prime}}=\left|\varepsilon \tau m\left(1+\frac{1}{M^{\alpha} C^{\alpha} \tau^{N \alpha}} m^{\alpha}\right)^{\frac{1}{\gamma^{\prime}}}\right|^{\gamma^{\prime}}=\varepsilon^{\gamma^{\prime}} \tau^{\gamma^{\prime}} m^{\gamma^{\prime}}\left(1+\frac{1}{M^{\alpha} C^{\alpha} \tau^{N \alpha}} m^{\alpha}\right) .
$$

We consider the energy at $(m, w)$

$$
\mathcal{E}(m, w)=\int_{\mathbb{R}^{N}} m L\left(-\frac{w}{m}\right)+F(m)+m V(x) d x
$$

Observe that by (1.3), $F(m) \leq-\frac{C_{f}}{\alpha+1} m^{\alpha+1}+K m$. Using Proposition 2.1, and computation (4.27) and (4.25), we get

$$
\begin{gathered}
\int_{\mathbb{R}^{N}} m L\left(-\frac{w}{m}\right)+F(m) d x \leq \int_{\mathbb{R}^{N}} m L\left(-\frac{w}{m}\right) d x-\frac{C_{f}}{\alpha+1} \int_{\mathbb{R}^{N}} m^{\alpha+1} d x+K M \\
\leq C_{L} \int_{\mathbb{R}^{N}} m \frac{|w| \gamma^{\gamma^{\prime}}}{m^{\gamma^{\prime}}} d x+\left(C_{L}+K\right) M-\frac{C_{f}}{\alpha+1} \int_{\mathbb{R}^{N}} m^{\alpha+1} d x \\
=C_{L} \varepsilon^{\gamma^{\prime}} \tau^{\gamma^{\prime}}\left(M+\int_{\mathbb{R}^{N}} \frac{1}{M^{\alpha} C^{\alpha} \tau^{N \alpha}} m^{\alpha+1} d x\right)+\left(C_{L}+K\right) M-\frac{C_{f}}{\alpha+1} \int_{\mathbb{R}^{N}} m^{\alpha+1} \\
=C_{L} \varepsilon^{\gamma^{\prime}} \tau^{\gamma^{\prime}} M+\left(C_{L}+K\right) M-\left(\frac{C_{f}}{\alpha+1}-\frac{\varepsilon^{\gamma^{\prime}} \tau^{\gamma^{\prime}-N \alpha}}{M^{\alpha} C^{\alpha}}\right) \int_{\mathbb{R}^{N}} m^{\alpha+1} d x \\
=\left(M C_{L}+M C C_{\alpha}\right) \varepsilon^{\gamma^{\prime}} \tau^{\gamma^{\prime}}-\frac{C_{f}}{\alpha+1} M^{\alpha+1} C^{\alpha+1} C_{\alpha} \tau^{\alpha N}+\left(C_{L}+K\right) M .
\end{gathered}
$$

We choose now $\tau$ such that $\tau=\frac{1}{A} \varepsilon^{-\frac{\gamma^{\prime}}{\gamma^{\prime}-N \alpha}}$, where $A$ is sufficiently large, in such a way that

$$
\int_{\mathbb{R}^{N}} m L\left(-\frac{w}{m}\right) d x+F(m) d x \leq-C \varepsilon^{-\frac{\gamma^{\prime} N \alpha}{\gamma^{\prime}-N \alpha}}+C
$$

where $C$ is a constant depending on $\alpha, C_{L}, M$. Substituting this in the energy and recalling (4.26), we get the desired inequality.

\section{Concentration phenomena}

In the second part of this work, we are interested in the asymptotic analysis of solutions to (1.1) when $\varepsilon \rightarrow 0$. 


\subsection{The rescaled problem}

We consider the following rescaling

$$
\left\{\begin{array}{l}
\tilde{m}(y):=\varepsilon^{\frac{N \gamma^{\prime}}{\gamma^{\prime}-\alpha N}} m\left(\varepsilon^{\frac{\gamma^{\prime}}{\gamma^{\prime}-\alpha N}} y\right), \\
\tilde{u}(y):=\varepsilon^{\frac{N \alpha\left(\gamma^{\prime}-1\right)-\gamma^{\prime}}{\gamma^{\prime}-\alpha N}} u\left(\varepsilon^{\frac{\gamma^{\prime}}{\gamma^{\prime}-\alpha N}} y\right) \\
\tilde{\lambda}:=\varepsilon^{\frac{N \alpha \gamma^{\prime}}{\gamma^{\prime}-\alpha N}} \lambda .
\end{array}\right.
$$

We introduce the rescaled potential

$$
V_{\varepsilon}(y)=\varepsilon^{\frac{N \alpha \gamma^{\prime}}{\gamma^{\prime}-\alpha N}} V\left(\varepsilon^{\frac{\gamma^{\prime}}{\gamma^{\prime}-\alpha N}} y\right)
$$

Note that by (1.5), we get

$$
C_{V}^{-1} \varepsilon^{\frac{N \alpha \gamma^{\prime}}{\gamma^{\prime}-\alpha N}}\left(\max \left\{\left|\varepsilon^{\frac{\gamma^{\prime}}{\gamma^{\prime}-\alpha N}} y\right|-C_{V}, 0\right\}\right)^{b} \leq V_{\varepsilon}(y) \leq C_{V} \varepsilon^{\frac{N \alpha \gamma^{\prime}}{\gamma^{\prime}-\alpha N}}\left(1+\varepsilon^{\frac{\gamma^{\prime}}{\gamma^{\prime}-\alpha N}}|y|\right)^{b} .
$$

The rescaled coupling term is given by

$$
f_{\varepsilon}(\tilde{m}(y))=\varepsilon^{\frac{N \alpha \gamma^{\prime}}{\gamma^{\prime}-\alpha N}} f\left(\varepsilon^{-\frac{N \gamma^{\prime}}{\gamma^{\prime}-\alpha N}} m\left(\varepsilon^{\frac{\gamma^{\prime}}{\gamma^{\prime}-\alpha N}} y\right)\right) .
$$

Note that, using (1.3), we obtain that

$$
-C_{f} m^{\alpha}-K \varepsilon^{\frac{N \alpha \gamma^{\prime}}{\gamma^{\prime}-\alpha N}} \leq f_{\varepsilon}(m) \leq-C_{f} m^{\alpha}+K \varepsilon^{\frac{N \alpha \gamma^{\prime}}{\gamma^{\prime}-\alpha N}},
$$

Then we get that

$$
\lim _{\varepsilon \rightarrow 0} f_{\varepsilon}(m)=-C_{f} m^{\alpha} \quad \text { uniformly in }[0,+\infty) .
$$

Moreover, we define $F_{\varepsilon}(m)=\int_{0}^{m} f_{\varepsilon}(n) d n$ if $m \geq 0$ and 0 otherwise, and we get

$$
-\frac{C_{f}}{\alpha+1} m^{\alpha+1}-K \varepsilon^{\frac{N \alpha \gamma^{\prime}}{\gamma^{\prime}-\alpha N}} m \leq F_{\varepsilon}(m) \leq-\frac{C_{f}}{\alpha+1} m^{\alpha+1}+K \varepsilon^{\frac{N \alpha \gamma^{\prime}}{\gamma^{\prime}-\alpha N}} m .
$$

We define also the rescaled Hamiltonian

$$
H_{\varepsilon}(p)=\varepsilon^{\frac{N \alpha \gamma^{\prime}}{\gamma^{\prime}-\alpha N}} H\left(\varepsilon^{-\frac{N \alpha\left(\gamma^{\prime}-1\right)}{\gamma^{\prime}-\alpha N}} p\right) .
$$

By (1.2),

$$
\begin{gathered}
C_{H}|p|^{\gamma}-\varepsilon^{\frac{N \alpha \gamma^{\prime}}{\gamma^{\prime}-\alpha N}} K \leq H_{\varepsilon}(p) \leq C_{H}|p|^{\gamma}, \\
\left|\nabla H_{\varepsilon}(p)\right| \leq K|p|^{\gamma-1} .
\end{gathered}
$$

So, we get that

$$
\lim _{\varepsilon \rightarrow 0} H_{\varepsilon}(p)=H_{0}(p):=C_{H}|p|^{\gamma} \quad \text { uniformly in } \mathbb{R}^{N} .
$$

Moreover, if we assume that $\nabla H_{\varepsilon}$ is locally bounded in $C^{0, \gamma-1}\left(\mathbb{R}^{N}\right)$, then

$$
\nabla H_{\varepsilon}(p) \rightarrow \nabla H_{0}(p)=\frac{C_{H}}{\gamma}|p|^{\gamma-2} p \quad \text { locally uniformly. }
$$

We can define $L_{\varepsilon}$ as in (1.7), with $H_{\varepsilon}$ in place of $H$ and we obtain that condition (5.9) gives that there exists $C_{L}>0$ such that

$$
C_{L}|q|^{\gamma^{\prime}} \leq L_{\varepsilon}(q) \leq C_{L}|q|^{\gamma^{\prime}}+\varepsilon^{\frac{N \alpha \gamma^{\prime}}{\gamma^{\prime}-\alpha N}} C_{L}
$$

which in turns gives that

$$
L_{\varepsilon}(q) \rightarrow L_{0}(q)=C_{L}|q|^{\gamma^{\prime}} \quad \text { uniformly in } \mathbb{R}^{N} .
$$


The rescalings (5.13) lead to the following rescaled system

$$
\left\{\begin{array}{l}
-\Delta \tilde{u}_{\varepsilon}+H_{\varepsilon}\left(\nabla \tilde{u}_{\varepsilon}\right)+\tilde{\lambda}_{\varepsilon}=f_{\varepsilon}\left(\tilde{m}_{\varepsilon}\right)+V_{\varepsilon}(y) \\
-\Delta \tilde{m}_{\varepsilon}-\operatorname{div}\left(\tilde{m}_{\varepsilon} \nabla H_{\varepsilon}\left(\nabla \tilde{u}_{\varepsilon}\right)\right)=0 \\
\int_{\mathbb{R}^{N}} \tilde{m}_{\varepsilon}=M
\end{array}\right.
$$

Existence of a triple $\left(\tilde{u}_{\varepsilon}, \tilde{m}_{\varepsilon}, \tilde{\lambda}_{\varepsilon}\right)$ solving the previous system is an immediate consequence of Theorem 1.1, We first start by stating some a priori estimates.

Lemma 5.1. There exist $C, C_{1}, C_{2}>0$ independent of $\varepsilon$ such that the following holds

$$
\begin{gathered}
-C_{1} \leq \tilde{\lambda}_{\varepsilon} \leq-C_{2}, \\
\int_{\mathbb{R}^{N}} \tilde{m}_{\varepsilon}\left|\nabla \tilde{u}_{\varepsilon}\right|^{\gamma} d y+\int_{\mathbb{R}^{N}} \tilde{m}_{\varepsilon}(y) V_{\varepsilon}(y) d y+\left\|\tilde{m}_{\varepsilon}\right\|_{L^{\alpha+1}\left(\mathbb{R}^{N}\right)}^{\alpha+1} \leq C, \\
\left\|\tilde{m}_{\varepsilon}\right\|_{L^{\infty}\left(\mathbb{R}^{N}\right)} \leq C .
\end{gathered}
$$

Proof. Estimates (4.23), (4.22) give (5.14), (5.15) by rescaling.

We apply Theorem 4.1 with $g[m](x)=f_{\varepsilon}(m(x)), r_{k}=\varepsilon^{\frac{N \alpha\left(\gamma^{\prime}-1\right)}{\gamma^{\prime}-\alpha N}}, s_{k}=\varepsilon^{\frac{N \alpha \gamma^{\prime}}{\gamma^{\prime}-\alpha N}}$ and $t_{k}=\varepsilon^{\frac{\gamma^{\prime}}{\gamma^{\prime}-\alpha N}}$, which are all bounded sequences, and we obtain (5.16).

Using the a priori bounds on the solutions to (5.13), we want to pass to the limit $\varepsilon \rightarrow 0$. The problem is that these estimates are not sufficient to assure that there is no loss of mass, namely that the limit of $\tilde{m}_{\varepsilon}$ has still $L^{1}$-norm equal to $M$. Therefore, we need to translate the reference system at a point around which the mass of $\tilde{m}_{\varepsilon}$ remains positive. This will be done as follows.

Let $y_{\varepsilon} \in \mathbb{R}^{N}$ be such that

$$
\tilde{u}_{\varepsilon}\left(y_{\varepsilon}\right)=\min _{\mathbb{R}^{N}} \tilde{u}_{\varepsilon}(y),
$$

note that this point exists due to (4.21).

We will denote by

$$
\left\{\begin{array}{l}
\bar{u}_{\varepsilon}(y)=\tilde{u}_{\varepsilon}\left(y+y_{\varepsilon}\right)-\tilde{u}_{\varepsilon}\left(y_{\varepsilon}\right) \\
\bar{m}_{\varepsilon}(y)=\tilde{m}_{\varepsilon}\left(y+y_{\varepsilon}\right) .
\end{array}\right.
$$

Note that $\left(\bar{u}_{\varepsilon}, \bar{m}_{\varepsilon}, \tilde{\lambda}_{\varepsilon}\right)$ is a classical solution to

$$
\left\{\begin{array}{l}
-\Delta \bar{u}_{\varepsilon}+H_{\varepsilon}\left(\nabla \bar{u}_{\varepsilon}\right)+\tilde{\lambda}_{\varepsilon}=f_{\varepsilon}\left(\bar{m}_{\varepsilon}\right)+V_{\varepsilon}\left(y+y_{\varepsilon}\right) \\
-\Delta \bar{m}_{\varepsilon}-\operatorname{div}\left(\bar{m}_{\varepsilon} \nabla H_{\varepsilon}\left(\nabla \bar{u}_{\varepsilon}\right)\right)=0 \\
\int_{\mathbb{R}^{N}} \bar{m}_{\varepsilon}=M
\end{array}\right.
$$

and in addition $\bar{u}_{\varepsilon}(0)=0=\min _{\mathbb{R}^{N}} \bar{u}_{\varepsilon}$.

\subsection{A preliminary convergence result}

In this section, we provide some preliminary convergence results, where we are not preventing possible loss of mass in the limit. First of all we need some a priori estimates on the solutions to (5.19).

Proposition 5.2. Let $\left(\bar{u}_{\varepsilon}, \bar{m}_{\varepsilon}, \tilde{\lambda}_{\varepsilon}\right)$ be as in (5.18). Then there exists a constant $C>0$ independent of $\varepsilon$ such that the following hold

$$
\begin{aligned}
& \varepsilon^{\frac{(N \alpha+b) \gamma^{\prime}}{\gamma^{\prime}-N \alpha}}\left|y_{\varepsilon}\right|^{b} \leq C \quad \text { and } \quad 0 \leq V_{\varepsilon}\left(y+y_{\varepsilon}\right) \leq C\left(\varepsilon^{\frac{(N \alpha+b) \gamma^{\prime}}{\gamma^{\prime}-N \alpha}}|y|^{b}+1\right) \text {, } \\
& \left|\nabla \bar{u}_{\varepsilon}(y)\right| \leq C(1+|y|)^{\frac{b}{\gamma}} \quad \text { and } \quad \bar{u}_{\varepsilon}(y) \geq C|y|^{1+\frac{b}{\gamma}}-C^{-1}, \\
& \int_{B_{R}(0)} \bar{m}_{\varepsilon}(y) d y \geq C \quad \forall R \geq 1 .
\end{aligned}
$$


Finally, if $\bar{w}_{\varepsilon}=-\bar{m}_{\varepsilon} \nabla H_{\varepsilon}\left(\nabla \bar{u}_{\varepsilon}\right)$, then $\left(\bar{m}_{\varepsilon}, \bar{w}_{\varepsilon}\right)$ is a minimizer in the set $\mathcal{K}_{1, M}$ of the energy

$$
\mathcal{E}_{\varepsilon}(m, w)=\int_{\mathbb{R}^{N}} m L_{\varepsilon}\left(-\frac{w}{m}\right)+V_{\varepsilon}\left(y+y_{\varepsilon}\right) m+F_{\varepsilon}(m) d y
$$

where $L_{\varepsilon}$ and $F_{\varepsilon}$ are defined in Section 5.1 .

Proof. Since $\bar{u}_{\varepsilon}$ is a classical solution, we can compute the equation in $y=0$, obtaining

$$
H_{\varepsilon}(0)+\tilde{\lambda}_{\varepsilon} \geq f_{\varepsilon}\left(\bar{m}_{\varepsilon}(0)\right)+V\left(y_{\varepsilon}\right) .
$$

Using the a priori estimates (5.14), (5.16), (5.9) and the assumption (5.5), (5.3), this implies that $\varepsilon^{\frac{(N \alpha+b) \gamma^{\prime}}{\gamma^{\prime}-N \alpha}}\left|y_{\varepsilon}\right|^{b} \leq C$, and then, again by assumption (5.3), that (5.20) holds.

Using estimates (5.14), (5.16), and (5.20), we conclude by Theorem 2.5 that estimate (5.21) holds.

Again by the equation computed at $y=0$, recalling that $H_{\varepsilon}(0) \rightarrow 0$ and $V_{\varepsilon} \geq 0$ and estimate (5.14), we deduce that $-f_{\varepsilon}\left(\bar{m}_{\varepsilon}(0)\right) \geq-C_{2}>0$. So, by assumption (5.5), we get that there exists $C>0$ indipendent of $\varepsilon$, such that $\tilde{m}_{\varepsilon}(0)>C>0$. Using the estimates (5.21) and (5.16), by Proposition 2.4 we get that there exists a positive constant depending on $p$ such that $\left\|\bar{m}_{\varepsilon}\right\|_{W^{1, p}\left(B_{2}(0)\right)} \leq C_{p}$ for all $p>1$. This, by Sobolev embeddings, gives that $\left\|\bar{m}_{\varepsilon}\right\|_{C^{0, \alpha}\left(B_{2}(0)\right)} \leq C_{\alpha}$ for every $\alpha \in(0,1)$ and for some positive constant depending on $\alpha$. We choose now $R_{0} \in(0,1]$ such that $\bar{m}_{\varepsilon} \geq C / 2$ in $B_{R_{0}}(0)$, using the $C^{\alpha}$ estimate and the fact that $\bar{m}_{\varepsilon}(0)>C>0$. This implies immediately that $\int_{B_{R_{0}}(0)} \bar{m}_{\varepsilon}(y) d y \geq C / 2\left|B_{R_{0}}\right|>0$. This gives the estimate (5.22), for all radii bigger than $R_{0}$.

Finally the fact that $\left(\bar{m}_{\varepsilon}, \bar{w}_{\varepsilon}\right)$ is a minimizer of (5.23) in $\mathcal{K}_{1, M}$ follows from Theorem 1.1] by rescaling.

We get the first convergence result.

Proposition 5.3. Let $\left(\bar{u}_{\varepsilon}, \bar{m}_{\varepsilon}, \tilde{\lambda}_{\varepsilon}\right)$ be the classical solution to (5.19) constructed above. Up to subsequences, we get that $\tilde{\lambda}_{\varepsilon} \rightarrow \bar{\lambda}$, and

$$
\bar{u}_{\varepsilon} \rightarrow \bar{u}, \quad \bar{m}_{\varepsilon} \rightarrow \bar{m}, \quad \nabla \bar{u}_{\varepsilon} \rightarrow \nabla \bar{u}, \quad \nabla H_{\varepsilon}\left(\nabla \bar{u}_{\varepsilon}\right) \rightarrow \nabla H_{0}(\nabla \bar{u})
$$

locally uniformly, where $\bar{u} \geq 0=\bar{u}(0)$, and $(\bar{u}, \bar{m}, \bar{\lambda})$ is a classical solution to

$$
\left\{\begin{array}{l}
-\Delta \bar{u}+H_{0}(\nabla \bar{u})+\bar{\lambda}=-C_{f} \bar{m}^{\alpha}+g(x) \\
-\Delta \bar{m}-\operatorname{div}\left(\bar{m} \nabla H_{0}(\nabla \bar{u})\right)=0
\end{array}\right.
$$

for a continuous function $g$ such that $0 \leq g(x) \leq C$ on $\mathbb{R}^{N}$ for some $C>0$.

Moreover, there exist $a \in(0, M], C, K, \kappa>0$ such that $\int_{\mathbb{R}^{N}} \bar{m} d x=a$, and

$$
\bar{u}(x) \geq C|x|-C, \quad|\nabla \bar{u}| \leq K \text { on } \mathbb{R}^{N}, \quad \int_{\mathbb{R}^{N}} e^{\kappa|x|} \bar{m}(x) d x<+\infty .
$$

Proof. First of all observe that, since $V$ is a locally Hölder continuous function, then (5.20) implies that, up to subsequence, $V_{\varepsilon}\left(x+y_{\varepsilon}\right) \rightarrow g(x)$, locally uniformly as $\varepsilon \rightarrow 0$, where $g$ is a continuous function such that $0 \leq g(x) \leq C$, for some $C>0$.

Using the a priori estimate (5.21), and recalling that $\bar{u}_{\varepsilon}$ is a classical solution to (5.19), by classical elliptic regularity theory we obtain that $\bar{u}_{\varepsilon}$ is locally bounded in $C^{1, \alpha}$ in every compact set, uniformly with respect to $\varepsilon$. So, up to extracting a subsequence via a diagonalization procedure, we get that

$$
\bar{u}_{\varepsilon} \rightarrow \bar{u}, \quad \nabla \bar{u}_{\varepsilon} \rightarrow \nabla \bar{u}, \quad \nabla H_{\varepsilon}\left(\nabla \bar{u}_{\varepsilon}\right) \rightarrow \nabla H_{0}(\nabla \bar{u})
$$

locally uniformly, and $\tilde{\lambda}_{\varepsilon} \rightarrow \bar{\lambda}$. Using the estimates (5.21) and (5.16), by Proposition 2.4, and by Sobolev embeddings, for every compact set $K \subset \mathbb{R}^{N}$, we have that $\left\|\bar{m}_{\varepsilon}\right\|_{C^{0, \alpha}(K)} \leq C_{K, \alpha}$ for 
every $\alpha \in(0,1)$ and for some positive constant depending on $\alpha$ and $K$. So, up to extracting a subsequence via a diagonalization procedure, we get that $\bar{m}_{\varepsilon} \rightarrow \bar{m}$ locally uniformly.

So, we can pass to the limit in (5.19) and obtain that $(\bar{u}, \bar{m}, \bar{\lambda})$ is a solution to (5.25), which is classical by elliptic regularity theory.

Using (5.22) and locally uniform convergence, we get that there exists $a \in(0, M]$ such that $\int_{\mathbb{R}^{N}} \bar{m} d y=a$.

Observe that $\bar{u}$ is a solution to

$$
-\Delta \bar{u}+H_{0}(\nabla \bar{u})+\bar{\lambda}=-C_{f} \bar{m}^{\alpha}+g(x) .
$$

By Theorem 2.5 we get that there exists a constant $K$ depending on $\sup g$ and $-\bar{\lambda}$ such that $|\nabla \bar{u}| \leq K$. Moreover, by construction $\bar{u} \geq 0$.

Since $\bar{m}$ is Hölder continuous, and such that $\int_{\mathbb{R}^{N}} \bar{m} d x=a \in(0, M]$, by Lemma 2.2, we get that $\bar{m} \rightarrow 0$ as $|x| \rightarrow+\infty$. Therefore, we get that $\liminf _{|x| \rightarrow+\infty}\left(-\bar{m}^{\alpha}(x)+g(x)-\bar{\lambda}-H_{0}(0)\right) \geq-\lambda>0$. So, by Theorem 2.6. recalling that by construction $\bar{u}(0)=0 \leq \bar{u}(y)$, we get that $\bar{u}$ satisfies

$$
\bar{u}(x) \geq C|x|-C
$$

for some $C>0$.

To conclude, consider the function $\Phi(x)=e^{\kappa \bar{u}(x)}$. We claim that we can choose $\kappa>0$ such that there exist $R>0$ and $\delta>0$ with

$$
-\Delta \Phi+\nabla H_{0}(\nabla \bar{u}) \cdot \nabla \Phi>\delta \Phi \quad|x|>R .
$$

Indeed, since $\bar{u}$ solves the first equation in (5.25), we get

$$
-\Delta \Phi+\nabla H_{0}(\nabla \bar{u}) \cdot \nabla \Phi \geq \kappa\left(-\bar{\lambda}-\kappa|\nabla \bar{u}|^{2}-\bar{m}^{\alpha}\right) \Phi .
$$

Using (5.27) and $\bar{m} \rightarrow 0$ as $|x| \rightarrow+\infty$, we obtain the claim. Reasoning as in [20, Proposition 4.3], or [28, Proposition 2.6], we get that $\int_{\mathbb{R}^{N}} e^{\kappa \bar{u}} \bar{m} d x<+\infty$, which concludes the estimate (5.26).

Remark 5.4. With estimates (5.26) in force, the pointwise bounds stated in [28, Theorem 6.1] hold, namely there exist positive constants $c_{1}, c_{2}$, such that

$$
\bar{m}(x) \leq c_{1} e^{-c_{2}|x|} \quad \text { on } \mathbb{R}^{N} .
$$

\subsection{Concentration-compactness}

In this section we show that actually there is no loss of mass when passing to the limit as in Proposition 5.3. In order to do so, we apply a kind of concentration-compactness argument.

First of all we show that the functional $\mathcal{E}_{\varepsilon}(m, w)$ enjoys the following subadditivity property. Let us denote

$$
\tilde{e}_{\varepsilon}(M)=\min _{(m, w) \in \mathcal{K}_{M}} \mathcal{E}_{\varepsilon}(m, w) .
$$

Recalling (3.6) and (4.24), and the rescaling (5.1), we get that for every $M>0$ there exist $C_{1}(M), C_{2}(M), K_{1}, K_{2}>0$ depending on $M$ (and on the other constants of the problem) but not on $\varepsilon$ such that there holds

$$
-C_{1}(M)-K_{1} \varepsilon^{\frac{N \alpha \gamma^{\prime}}{\gamma^{\prime}-N \alpha}} \leq \tilde{e}_{\varepsilon}(M) \leq-C_{2}(M)-K_{2} \varepsilon^{\frac{N \alpha \gamma^{\prime}}{\gamma^{\prime}-N \alpha}} .
$$

Lemma 5.5. For all $a \in(0, M)$, there exist $\varepsilon_{0}=\varepsilon_{0}(a)$ and a constant $C=C(a, M) \geq 0$ depending only on $a, M$ and the data (not on $\varepsilon$ ), such that $C(M, M)=0=C(0, M), C(a, M)>0$ for $0<a<M$ and

$$
\tilde{e}_{\varepsilon}(M) \leq \tilde{e}_{\varepsilon}(a)+\tilde{e}_{\varepsilon}(M-a)-C(a, M) \quad \forall \varepsilon \leq \varepsilon_{0} .
$$


Proof. We assume that $a \geq M / 2$ (otherwise it suffices to replace $a$ with $M-a$ ).

Let $c>1$ and $B>0$. For all admissible couples $(m, w) \in \mathcal{K}_{B}$ we have, recalling (5.7),

$$
\begin{array}{rl}
\tilde{e}_{\varepsilon}(c B) \leq \mathcal{E}_{\varepsilon}(c m, c w)=\int_{\mathbb{R}^{N}} & c m L_{\varepsilon}\left(-\frac{w}{m}\right)+F_{\varepsilon}(c m)+c V_{\varepsilon}\left(x+y_{\varepsilon}\right) m d x \\
= & c \mathcal{E}_{\varepsilon}(m, w)+\int_{\mathbb{R}^{N}} F_{\varepsilon}(c m)-c F_{\varepsilon}(m) d x \\
& \leq c \mathcal{E}_{\varepsilon}(m, w)-\frac{c\left(c^{\alpha}-1\right) C_{f}}{\alpha+1} \int_{\mathbb{R}^{N}} m^{\alpha+1} d x+2 K c B \varepsilon^{\frac{N \alpha \gamma^{\prime}}{\gamma^{\prime}-N \alpha}}
\end{array}
$$

Let now $\left(m_{n}, w_{n}\right)$ be a minimizing sequence of $\mathcal{E}_{\varepsilon}$ in $\mathcal{K}_{B}$, such that $\mathcal{E}_{\varepsilon}\left(m_{n}, w_{n}\right) \leq \tilde{e}_{\varepsilon}(B)+\frac{C_{2}(B)}{4}$ where $C_{2}(B)$ is the constant appearing in (5.29), which depends on $B$ and on the data of the problem. Recalling that $V_{\varepsilon} \geq 0$ and $L_{\varepsilon} \geq 0$, and estimate (5.7), we get that

$$
\tilde{e}_{\varepsilon}(M)+\frac{C_{2}(B)}{4} \geq \mathcal{E}_{\varepsilon}\left(m_{n}, w_{n}\right) \geq \int_{\mathbb{R}^{N}} F_{\varepsilon}\left(m_{n}\right) d x \geq-\frac{C_{f}}{\alpha+1} \int_{\mathbb{R}^{n}} m^{\alpha+1} d x-K B \varepsilon^{\frac{N \alpha \gamma^{\prime}}{\gamma^{\prime}-N \alpha}} .
$$

Using (5.29), we get, for all $\varepsilon$ sufficiently small,

$$
\frac{C_{f}}{\alpha+1} \int_{\mathbb{R}^{N}} m_{n}^{\alpha+1} d x \geq \frac{3 C_{2}(B)}{4}-K \varepsilon^{\frac{N \alpha \gamma^{\prime}}{\gamma^{\prime}-\alpha N}}>\frac{C_{2}(B)}{2}>0 .
$$

So, this estimate in particular holds for a minimizer of $\mathcal{E}_{\varepsilon}$. Therefore in (5.31) we get, taking $(m, w)$ to be a minimizer of $\mathcal{E}_{\varepsilon}$ (which exists by Proposition 5.2)

$$
\tilde{e}_{\varepsilon}(c B)<c \tilde{e}_{\varepsilon}(B)-c\left(c^{\alpha}-1\right) \frac{C_{2}(B)}{2}+2 K c B \varepsilon^{\frac{N \alpha \gamma^{\prime}}{\gamma^{\prime}-N \alpha}} .
$$

Using (5.32) with $B=a$ and $c=M / a$ we get

$$
\tilde{e}_{\varepsilon}(M)<\frac{M}{a} \tilde{e}_{\varepsilon}(a)-\frac{M}{a}\left[\left(\frac{M}{a}\right)^{\alpha}-1\right] \frac{C_{2}(a)}{2}+2 K M \varepsilon^{\frac{N \alpha \gamma^{\prime}}{\gamma^{\prime}-N \alpha}} .
$$

If $a=M / 2$, this permits to conclude, choosing $\varepsilon$ sufficiently small (depending on $a$ ). If $a>M / 2$, we use (5.32) with $B=M-a$ and $c=a /(M-a)$ to get (multiplying everything by $\frac{M-a}{a}$ )

$$
\begin{aligned}
& \frac{M-a}{a} \tilde{e}_{\varepsilon}(a)<\tilde{e}_{\varepsilon}(M-a)-\left[\left(\frac{a}{M-a}\right)^{\alpha}-1\right] \frac{C_{2}(M-a)}{2}+2 K(M-a) \varepsilon^{\frac{N \alpha \gamma^{\prime}}{\gamma^{\prime}-N \alpha}} \\
& \quad<\tilde{e}_{\varepsilon}(M-a)-\left[\left(\frac{a}{M-a}\right)^{\alpha}-1\right] \frac{C_{2}(M-a)}{2}+2 K M \varepsilon^{\frac{N \alpha \gamma^{\prime}}{\gamma^{\prime}-N \alpha}} \leq \tilde{e}_{\varepsilon}(M-a)+2 K M \varepsilon^{\frac{N \alpha \gamma^{\prime}}{\gamma^{\prime}-N \alpha}}
\end{aligned}
$$

So putting to together the last two inequalities we get

$$
\begin{aligned}
\tilde{e}_{\varepsilon}(M)<\frac{M}{a} \tilde{e}_{\varepsilon}(a)-\frac{M}{a}\left[\left(\frac{M}{a}\right)^{\alpha}-1\right] & \frac{C_{2}(a)}{2}+2 K M \varepsilon^{\frac{N \alpha \gamma^{\prime}}{\gamma^{\prime}-N \alpha}} \\
= & \tilde{e}_{\varepsilon}(a)+\frac{M-a}{a} \tilde{e}_{\varepsilon}(a)-\frac{M}{a}\left[\left(\frac{M}{a}\right)^{\alpha}-1\right] \frac{C_{2}(a)}{2}+2 K M \varepsilon^{\frac{N \alpha \gamma^{\prime}}{\gamma^{\prime}-N \alpha}} \\
< & \tilde{e}_{\varepsilon}(a)+\tilde{e}_{\varepsilon}(M-a)-\frac{M}{a}\left[\left(\frac{M}{a}\right)^{\alpha}-1\right] \frac{C_{2}(a)}{2}+4 K M \varepsilon^{\frac{N \alpha \gamma^{\prime}}{\gamma^{\prime}-N \alpha}} \\
& \quad \leq \tilde{e}_{\varepsilon}(a)+\tilde{e}_{\varepsilon}(M-a)-\frac{M}{a}\left[\left(\frac{M}{a}\right)^{\alpha}-1\right] \frac{C_{2}(a)}{4}
\end{aligned}
$$

for $\varepsilon$ sufficiently small (depending on $a$ ). 
Theorem 5.6. Let $\left(\bar{m}_{\varepsilon}, \bar{w}_{\varepsilon}\right)$ be the minimizer of $\mathcal{E}_{\varepsilon}$ as in Proposition 5.2. Let $\bar{u}, \bar{m}$ as in Proposition [5.3, so that $\bar{m}_{\varepsilon} \rightarrow \bar{m}, \bar{w}_{\varepsilon} \rightarrow \bar{w}=-\bar{m} \nabla H_{0}(\nabla \bar{u})$ locally uniformly, and $\bar{m}$ satisfies the exponential decay (5.26). Then,

$$
\int_{\mathbb{R}^{N}} \bar{m} d x=M
$$

Proof. Assume by contradiction that $\int_{\mathbb{R}^{N}} \bar{m} d x=a$, with $0<a<M$. We fix $\varepsilon_{0}(a)$ as in Lemma 5.5. and we consider from now on $\varepsilon \leq \varepsilon_{0}(a)$. Let $\bar{c}>0$ be such that $\bar{m} \leq \bar{c} e^{-|x|}$ (such $\bar{c}$ exists by Remark 5.4).

For $R$ sufficiently large (to be chosen later), we define

$$
\nu_{R}(x)= \begin{cases}\bar{c} e^{-R} & |x| \leq R \\ \bar{c} e^{-|x|} & |x|>R .\end{cases}
$$

So in particular $\bar{m}(x) \leq \nu_{R}(x)$ for $|x|>R$.

We observe that as $R \rightarrow+\infty$

$$
\int_{\mathbb{R}^{n}} \nu_{R}(x) d x=\bar{c} \omega_{N} e^{-R} R^{N}+\int_{\mathbb{R}^{N} \backslash B_{R}} \bar{c} e^{-|x|} d x \leq C e^{-R} R^{N} \rightarrow 0 .
$$

Since $\bar{m}_{\varepsilon} \rightarrow \bar{m}$ and $\nabla H_{\varepsilon}\left(\nabla \bar{u}_{\varepsilon}\right) \rightarrow \nabla H_{0}(\nabla \bar{u})$ locally uniformly, there exists $\varepsilon_{0}=\varepsilon_{0}(R)$ such that for all $\varepsilon \leq \varepsilon_{0}$,

$$
\left|\bar{m}_{\varepsilon}-\bar{m}\right|+\left|\nabla H_{\varepsilon}\left(\nabla \bar{u}_{\varepsilon}\right)-\nabla H_{0}(\nabla \bar{u})\right| \leq \bar{c} e^{-R} \quad|x| \leq R .
$$

We observe that for all $\varepsilon \leq \varepsilon_{0}$,

$$
\bar{m}_{\varepsilon}-\bar{m}+2 \nu_{R} \geq \nu_{R}(x) \quad \forall x \in \mathbb{R}^{N} .
$$

Indeed, if $|x|>R$, then $\bar{m}_{\varepsilon}-\bar{m}+2 \nu_{R} \geq \bar{m}_{\varepsilon}+\nu_{R} \geq \nu_{R}$, since $\bar{m} \leq \nu_{R}$. On the other hand, if $|x| \leq R$, then by (5.36) $\bar{m}_{\varepsilon}-\bar{m}+2 \nu_{R} \geq-\bar{c} e^{-R}+2 \bar{c} e^{-R}=\bar{c} e^{-R}=\nu_{R}$. From (5.37) we deduce that

$$
\left|\bar{m}_{\varepsilon}-\bar{m}\right| \leq \bar{m}_{\varepsilon}-\bar{m}+2 \nu_{R}
$$

Moreover, since $\bar{m}_{\varepsilon} \rightarrow \bar{m}$ a.e. by Theorem 2.3, recalling that $\int_{\mathbb{R}^{N}} \bar{m}_{\varepsilon} d x=M, \int_{\mathbb{R}^{n}} \bar{m}=a$ and using (5.35) and (5.38), we have that

$$
\begin{aligned}
\int_{\mathbb{R}^{N}}\left(\bar{m}_{\varepsilon}-\bar{m}+2 \nu_{R}\right) d x & =M-a+2 \int_{\mathbb{R}^{N}} \nu_{R} d x \rightarrow M-a \quad \text { as } R \rightarrow+\infty, \\
\lim _{\varepsilon \rightarrow 0} \int_{\mathbb{R}^{N}} \bar{m}_{\varepsilon}^{\alpha+1} d x & =\int_{\mathbb{R}^{N}} \bar{m}^{\alpha+1} d x+\lim _{\varepsilon \rightarrow 0} \int_{\mathbb{R}^{N}}\left|\bar{m}_{\varepsilon}-\bar{m}\right|^{\alpha+1} d x \\
& \leq \int_{\mathbb{R}^{N}} \bar{m}^{\alpha+1} d x+\lim _{\varepsilon \rightarrow 0} \int_{\mathbb{R}^{N}}\left(\bar{m}_{\varepsilon}-\bar{m}+2 \nu_{R}\right)^{\alpha+1} d x
\end{aligned}
$$

We claim that

$$
\mathcal{E}_{\varepsilon}\left(\bar{m}_{\varepsilon}, \bar{w}_{\varepsilon}\right) \geq \mathcal{E}_{\varepsilon}(\bar{m}, \bar{w})+\mathcal{E}_{\varepsilon}\left(\bar{m}_{\varepsilon}-\bar{m}+2 \nu_{R}, \bar{w}_{\varepsilon}-\bar{w}+2 \nabla \nu_{R}\right)+o_{\varepsilon}(1)+o_{R}(1),
$$

where $o_{\varepsilon}(1)$ is an error such that $\lim _{\varepsilon \rightarrow 0} o_{\varepsilon}(1)=0$.

We consider the function $(m, w) \mapsto m L_{\varepsilon}\left(-\frac{w}{m}\right)$. This is a convex function in $(m, w)$. We compute $\nabla_{w}\left(m L_{\varepsilon}\left(-\frac{w}{m}\right)\right)=-\nabla L_{\varepsilon}\left(-\frac{w}{m}\right)$, so in particular by (5.11) we get

$$
C_{L}\left|\frac{w}{m}\right|^{\gamma^{\prime}-1}-C_{L}^{-1} \varepsilon^{\frac{N \alpha\left(\gamma^{\prime}-1\right)}{\gamma^{\prime}-\alpha N}} \leq\left|\nabla_{w}\left(m L_{\varepsilon}\left(-\frac{w}{m}\right)\right)\right| \leq C_{L}^{-1}\left|\frac{w}{m}\right|^{\gamma^{\prime}-1}+C_{L}^{-1} \varepsilon^{\frac{N \alpha\left(\gamma^{\prime}-1\right)}{\gamma^{\prime}-\alpha N}} .
$$

Moreover, $\partial_{m}\left(m L_{\varepsilon}\left(-\frac{w}{m}\right)\right)=L_{\varepsilon}\left(-\frac{w}{m}\right)+\frac{w}{m} \cdot \nabla L_{\varepsilon}\left(-\frac{w}{m}\right)$, therefore, again by (5.11) we get

$$
C_{L}\left|\frac{w}{m}\right|^{\gamma^{\prime}}-C_{L}^{-1} \varepsilon^{\frac{N \alpha\left(\gamma^{\prime}-1\right)}{\gamma^{\prime}-\alpha N}} \leq\left|\partial_{m}\left(m L_{\varepsilon}\left(-\frac{w}{m}\right)\right)\right| \leq C_{L}^{-1}\left|\frac{w}{m}\right|^{\gamma^{\prime}}+C_{L}^{-1} \varepsilon^{\frac{N \alpha\left(\gamma^{\prime}-1\right)}{\gamma^{\prime}-\alpha N}} .
$$


Note that

$\int_{\mathbb{R}^{N}} V_{\varepsilon}\left(y+y_{\varepsilon}\right) \bar{m}_{\varepsilon} d x=\int_{\mathbb{R}^{N}} V_{\varepsilon}\left(y+y_{\varepsilon}\right) \bar{m} d x+\int_{\mathbb{R}^{N}} V_{\varepsilon}\left(y+y_{\varepsilon}\right)\left(\bar{m}_{\varepsilon}-\bar{m}+2 \nu_{R}\right) d x-2 \int_{\mathbb{R}^{N}} V_{\varepsilon}\left(y+y_{\varepsilon}\right) \nu_{R} d x$.

Recalling the estimate (5.20) and the definition of $\nu_{R}$, we have

$$
2 \int_{\mathbb{R}^{N}} V_{\varepsilon}\left(y+y_{\varepsilon}\right) \nu_{R} d x \leq C R^{b+N} e^{-R} .
$$

Hence we obtain

$$
\int_{\mathbb{R}^{N}} V_{\varepsilon}\left(y+y_{\varepsilon}\right) \bar{m}_{\varepsilon} d x \geq \int_{\mathbb{R}^{N}} V_{\varepsilon}\left(y+y_{\varepsilon}\right) \bar{m} d x+\int_{\mathbb{R}^{N}} V_{\varepsilon}\left(y+y_{\varepsilon}\right)\left(\bar{m}_{\varepsilon}-\bar{m}+2 \nu_{R}\right) d x-C R^{b+N} e^{-R} .
$$

By (5.40) and (5.7) we get

$$
\begin{aligned}
& \int_{\mathbb{R}^{N}} F_{\varepsilon}\left(\bar{m}_{\varepsilon}\right) d x \geq-\frac{C_{f}}{\alpha+1} \int_{\mathbb{R}^{N}} \bar{m}_{\varepsilon}^{\alpha+1} d x-K M \varepsilon^{\frac{N \alpha \gamma^{\prime}}{\gamma^{\prime}-\alpha N}} \\
& \geq-\frac{C_{f}}{\alpha+1} \int_{\mathbb{R}^{N}} \bar{m}^{\alpha+1} d x-\frac{C_{f}}{\alpha+1} \int_{\mathbb{R}^{N}}\left(\bar{m}_{\varepsilon}-\bar{m}+2 \nu_{R}\right)^{\alpha+1} d x+o_{\varepsilon}(1) \\
& \geq \int_{\mathbb{R}^{N}} F_{\varepsilon}(\bar{m}) d x+\int_{\mathbb{R}^{N}} F_{\varepsilon}\left(\bar{m}_{\varepsilon}-\bar{m}+2 \nu_{R}\right) d x+o_{\varepsilon}(1) .
\end{aligned}
$$

Finally, we estimate the kinetic terms in the energy. Splitting

$$
\int_{\mathbb{R}^{N}} \bar{m}_{\varepsilon} L_{\varepsilon}\left(-\frac{\bar{w}_{\varepsilon}}{\bar{m}_{\varepsilon}}\right) d x=\int_{B_{R}} \bar{m}_{\varepsilon} L_{\varepsilon}\left(-\frac{\bar{w}_{\varepsilon}}{\bar{m}_{\varepsilon}}\right) d x+\int_{\mathbb{R}^{N} \backslash B_{R}} \bar{m}_{\varepsilon} L\left(-\frac{\bar{w}_{\varepsilon}}{\bar{m}_{\varepsilon}}\right) d x,
$$

we proceed by estimating separately the two terms.

Estimates in $\mathbb{R}^{N} \backslash B_{R}$.

First of all, note that by (5.26), (5.9) and (5.11), we get that $L_{\varepsilon}\left(-\frac{\bar{w}}{\bar{m}}\right)=L_{\varepsilon}\left(\nabla H_{0}(\nabla \bar{u})\right) \leq C$ for come constant $C>0$, just depending on the data. Moreover, recalling that $\bar{m} \leq \bar{c} e^{-|x|}$, we get that, eventually enlarging $C$,

$$
\int_{\mathbb{R}^{N} \backslash B_{R}} \bar{m} L_{\varepsilon}\left(-\frac{\bar{w}}{\bar{m}}\right) d x \leq C \int_{|x|>R} e^{-|x|} d x \leq C R^{N} e^{-R} .
$$

By convexity of the function $(m, w) \mapsto m L\left(-\frac{w}{m}\right)$, we get that

$$
\begin{array}{r}
\int_{\mathbb{R}^{N} \backslash B_{R}} \bar{m}_{\varepsilon} L\left(-\frac{\bar{w}_{\varepsilon}}{\bar{m}_{\varepsilon}}\right) d x \geq \int_{\mathbb{R}^{N} \backslash B_{R}}\left(\bar{m}_{\varepsilon}-\bar{m}+2 \nu_{R}\right) L_{\varepsilon}\left(-\frac{\bar{w}_{\varepsilon}-\bar{w}+2 \nabla \nu_{R}}{\bar{m}_{\varepsilon}-\bar{m}+2 \nu_{R}}\right) d x \\
\quad+\int_{\mathbb{R}^{N} \backslash B_{R}} \partial_{m}\left(\left(\bar{m}_{\varepsilon}-\bar{m}+2 \nu_{R}\right) L_{\varepsilon}\left(-\frac{\bar{w}_{\varepsilon}-\bar{w}+2 \nabla \nu_{R}}{\bar{m}_{\varepsilon}-\bar{m}+2 \nu_{R}}\right)\right)\left(\bar{m}-2 \nu_{R}\right) d x \\
+\int_{\mathbb{R}^{N} \backslash B_{R}} \nabla_{w}\left[\left(\bar{m}_{\varepsilon}-\bar{m}+2 \nu_{R}\right) L_{\varepsilon}\left(-\frac{\bar{w}_{\varepsilon}-\bar{w}+2 \nabla \nu_{R}}{\bar{m}_{\varepsilon}-\bar{m}+2 \nu_{R}}\right)\right] \cdot\left(\bar{w}-2 \nabla \nu_{R}\right) d x .
\end{array}
$$

We recall that $|\bar{w}|=\bar{m}\left|\nabla H_{0}(\nabla \bar{u})\right| \leq C \bar{m}$ by (5.26) and $\left|\nabla \nu_{R}\right| \leq C \nu_{R}$ by definition. Moreover, by (5.21) and (5.9),

$$
\left|\bar{w}_{\varepsilon}\right|=\bar{m}_{\varepsilon}\left|\nabla H_{\varepsilon}\left(\nabla \bar{u}_{\varepsilon}\right)\right| \leq C \bar{m}_{\varepsilon}\left[(1+|x|)^{\frac{b}{\gamma}}\right]^{\gamma-1} \leq C_{1} \bar{m}_{\varepsilon}(1+|x|)^{\frac{b}{\gamma^{\prime}}} .
$$

Using the triangular inequality we get the following, where the constant $C$ can change from line to line,

$$
\begin{aligned}
& \left|\frac{\bar{w}_{\varepsilon}-\bar{w}+2 \nabla \nu_{R}}{\bar{m}_{\varepsilon}-\bar{m}+2 \nu_{R}}\right| \leq \frac{\bar{m}_{\varepsilon}\left|\nabla H_{\varepsilon}\left(\nabla \bar{u}_{\varepsilon}\right)\right|}{\bar{m}_{\varepsilon}-\bar{m}+2 \nu_{R}}+\frac{\bar{m}\left|\nabla H_{0}(\nabla \bar{u})\right|}{\bar{m}_{\varepsilon}-\bar{m}+2 \nu_{R}}+\frac{C \nu_{R}}{\bar{m}_{\varepsilon}-\bar{m}+2 \nu_{R}} \\
& \quad \leq \frac{C \bar{m}_{\varepsilon}(1+|x|)^{\frac{b}{\gamma^{\prime}}}}{\bar{m}_{\varepsilon}-\bar{m}+2 \nu_{R}}+\frac{C \bar{m}}{\bar{m}_{\varepsilon}-\bar{m}+2 \nu_{R}}+\frac{C \nu_{R}}{\bar{m}_{\varepsilon}-\bar{m}+2 \nu_{R}} \leq C(1+|x|)^{\frac{b}{\gamma^{\prime}}}
\end{aligned}
$$


on $\mathbb{R}^{N} \backslash B_{R}(0)$, where we used respectively the fact that $\bar{m}_{\varepsilon}-\bar{m}+2 \nu_{R} \geq \bar{m}_{\varepsilon}, \bar{m} \leq \nu_{R}$ and that $\bar{m}_{\varepsilon}-\bar{m}+2 \nu_{R} \geq \nu_{R}$.

Now, using (5.43) and (5.49), we can estimate (5.47), and by (5.42) and (5.49) we can estimate (5.48). Indeed, we get

$\int_{\mathbb{R}^{N} \backslash B_{R}}\left|\partial_{m}\left(\left(\bar{m}_{\varepsilon}-\bar{m}+2 \nu_{R}\right) L_{\varepsilon}\left(-\frac{\bar{w}_{\varepsilon}-\bar{w}+2 \nabla \nu_{R}}{\bar{m}_{\varepsilon}-\bar{m}+2 \nu_{R}}\right)\right)\right|\left|\bar{m}-2 \nu_{R}\right| d x \leq C \int_{\mathbb{R}^{N} \backslash B_{R}}(1+|x|)^{b} \nu_{R}(x) d x$

and

$\int_{\mathbb{R}^{N} \backslash B_{R}}\left|\nabla_{w}\left[\left(\bar{m}_{\varepsilon}-\bar{m}+2 \nu_{R}\right) L_{\varepsilon}\left(-\frac{\bar{w}_{\varepsilon}-\bar{w}+2 \nabla \nu_{R}}{\bar{m}_{\varepsilon}-\bar{m}+2 \nu_{R}}\right)\right]\right|\left(|\bar{w}|+2\left|\nabla \nu_{R}\right|\right) d x \leq C \int_{\mathbb{R}^{N} \backslash B_{R}}(1+|x|)^{\frac{b}{\gamma}} \nu_{R}(x) d x$,

because $\bar{w} \leq C \bar{m}$ on $\mathbb{R}^{N}$. Therefore, we may conclude, possibly enlarging $C$, that

$$
\begin{aligned}
& \int_{\mathbb{R}^{N} \backslash B_{R}} \bar{m}_{\varepsilon} L\left(-\frac{\bar{w}_{\varepsilon}}{\bar{m}_{\varepsilon}}\right) d x \\
& \geq \int_{\mathbb{R}^{N} \backslash B_{R}}\left(\bar{m}_{\varepsilon}-\bar{m}+2 \nu_{R}\right) L_{\varepsilon}\left(-\frac{\bar{w}_{\varepsilon}-\bar{w}+2 \nabla \nu_{R}}{\bar{m}_{\varepsilon}-\bar{m}+2 \nu_{R}}\right) d x-C \int_{\mathbb{R}^{N} \backslash B_{R}}(1+|x|)^{b} \nu_{R}(x) d x \\
& \quad \geq \int_{\mathbb{R}^{N} \backslash B_{R}}\left(\bar{m}_{\varepsilon}-\bar{m}+2 \nu_{R}\right) L_{\varepsilon}\left(-\frac{\bar{w}_{\varepsilon}-\bar{w}+2 \nabla \nu_{R}}{\bar{m}_{\varepsilon}-\bar{m}+2 \nu_{R}}\right) d x-C R^{N+b} e^{-R}
\end{aligned}
$$

Finally, putting together (5.46) and (5.50), we have, choosing $C$ suffficiently large

$$
\begin{aligned}
\int_{\mathbb{R}^{N} \backslash B_{R}} \bar{m}_{\varepsilon} L_{\varepsilon}(- & \left.\frac{\bar{w}_{\varepsilon}}{\bar{m}_{\varepsilon}}\right) d x \geq \int_{\mathbb{R}^{N} \backslash B_{R}} \bar{m} L_{\varepsilon}\left(-\frac{\bar{w}}{\bar{m}}\right) d x \\
& +\int_{\mathbb{R}^{N} \backslash B_{R}}\left(\bar{m}_{\varepsilon}-\bar{m}+2 \nu_{R}\right) L_{\varepsilon}\left(-\frac{\bar{w}_{\varepsilon}-\bar{w}+2 \nabla \nu_{R}}{\bar{m}_{\varepsilon}-\bar{m}+2 \nu_{R}}\right) d x-C R^{N+b} e^{-R} .
\end{aligned}
$$

Estimates in $B_{R}$. Again by convexity of the function $(m, w) \mapsto m L\left(-\frac{w}{m}\right)$, we get that

$$
\begin{aligned}
\int_{B_{R}} \bar{m}_{\varepsilon} L & \left(-\frac{\bar{w}_{\varepsilon}}{\bar{m}_{\varepsilon}}\right) d x \geq \int_{B_{R}} \bar{m} L_{\varepsilon}\left(-\frac{\bar{w}}{\bar{m}}\right) d x \\
& +\int_{B_{R}} \partial_{m}\left(\bar{m} L_{\varepsilon}\left(-\frac{\bar{w}}{\bar{m}}\right)\right)\left(\bar{m}_{\varepsilon}-\bar{m}\right) d x+\int_{B_{R}} \nabla_{w}\left[\bar{m} L_{\varepsilon}\left(-\frac{\bar{w}}{\bar{m}}\right)\right] \cdot\left(\bar{w}_{\varepsilon}-\bar{w}\right) d x .
\end{aligned}
$$

We now estimate (5.52). We recall that $\left|\frac{\bar{w}}{\bar{m}}\right| \leq\left|\nabla H_{0}(\nabla \bar{u})\right| \leq K$ and also $\left|\nabla H_{\varepsilon}\left(\nabla \bar{u}_{\varepsilon}\right)\right| \leq K$ for all $\varepsilon \leq \varepsilon_{0}(R)$. Then, using this fact and (5.42) and (5.43) and recalling (5.36), we get

$$
\int_{B_{R}}\left|\partial_{m}\left(\bar{m} L_{\varepsilon}\left(-\frac{\bar{w}}{\bar{m}}\right)\right)\right|\left|\bar{m}_{\varepsilon}-\bar{m}\right| d x=\int_{B_{R}}\left|\partial_{m}\left(\bar{m} L_{\varepsilon}\left(\nabla H_{0}(\nabla \bar{u})\right)\right)\right|\left|\bar{m}_{\varepsilon}-\bar{m}\right| d x \leq C e^{-R} R^{N}
$$

and

$\int_{B_{R}}\left|\nabla_{w}\left[\bar{m} L_{\varepsilon}\left(\nabla H_{0}(\nabla \bar{u})\right)\right]\right|\left(\left|\nabla H_{\varepsilon}\left(\nabla u_{\varepsilon}\right)\right|\left|\bar{m}_{\varepsilon}-\bar{m}\right|+\left|\nabla H_{\varepsilon}\left(\nabla \bar{u}_{\varepsilon}\right)-\nabla H_{0}(\nabla \bar{u})\right| \bar{m}\right) d x \leq C e^{-R} R^{N}$.

This implies that for all $\varepsilon \leq \varepsilon_{0}(R)$

$$
\int_{B_{R}} \bar{m}_{\varepsilon} L\left(-\frac{\bar{w}_{\varepsilon}}{\bar{m}_{\varepsilon}}\right) d x \geq \int_{B_{R}} \bar{m} L_{\varepsilon}\left(-\frac{\bar{w}}{\bar{m}}\right) d x-C e^{-R} R^{N} .
$$

Now we observe that by (5.11),

$$
\int_{B_{R}}\left(\bar{m}_{\varepsilon}-\bar{m}+2 \nu_{R}\right) L_{\varepsilon}\left(-\frac{\bar{w}_{\varepsilon}-\bar{w}+2 \nabla \nu_{R}}{\bar{m}_{\varepsilon}-\bar{m}+2 \nu_{R}}\right) d x \leq C \int_{B_{R}}\left[\left|\frac{\bar{w}_{\varepsilon}-\bar{w}+2 \nabla \nu_{R}}{\bar{m}_{\varepsilon}-\bar{m}+2 \nu_{R}}\right|^{\gamma^{\prime}}+1\right]\left(\bar{m}_{\varepsilon}-\bar{m}+2 \nu_{R}\right) d x .
$$


By (5.38) we get that $\bar{m}_{\varepsilon}-\bar{m}+2 \nu_{R} \leq\left|\bar{m}_{\varepsilon}-\bar{m}\right|+2 \nu_{R} \leq C e^{-R}$, eventually enalarging $C$. Moreover, reasoning as in (5.49), we get

$$
\left|\frac{\bar{w}_{\varepsilon}-\bar{w}+2 \nabla \nu_{R}}{\bar{m}_{\varepsilon}-\bar{m}+2 \nu_{R}}\right| \leq\left|\nabla H_{\varepsilon}\left(\nabla \bar{u}_{\varepsilon}\right)\right| \frac{\left|\bar{m}_{\varepsilon}-\bar{m}\right|}{\bar{m}_{\varepsilon}-\bar{m}+2 \nu_{R}}+\frac{\left|\nabla H_{\varepsilon}\left(\nabla \bar{u}_{\varepsilon}\right)-\nabla H_{0}(\nabla \bar{u})\right|}{\bar{m}_{\varepsilon}-\bar{m}+2 \nu_{R}} \bar{m} \leq C
$$

where we used that $\nabla \nu_{R}=0$ for $|x|<R$, that $\left|\nabla H_{\varepsilon}\left(\nabla \bar{u}_{\varepsilon}\right)\right| \leq K$, that by (5.38) $\frac{\left|\bar{m}_{\varepsilon}-\bar{m}\right|}{\bar{m}_{\varepsilon}-\bar{m}+2 \nu_{R}} \leq 1$, $\frac{\left|\nabla H_{\varepsilon}\left(\nabla \bar{u}_{\varepsilon}\right)-\nabla H_{0}(\nabla \bar{u})\right|}{\bar{m}_{\varepsilon}-\bar{m}+2 \nu_{R}} \leq C$ by (5.37) and (5.36). So, we conclude that

$$
\int_{B_{R}}\left(\bar{m}_{\varepsilon}-\bar{m}+2 \nu_{R}\right) L_{\varepsilon}\left(-\frac{\bar{w}_{\varepsilon}-\bar{w}+2 \nabla \nu_{R}}{\bar{m}_{\varepsilon}-\bar{m}+2 \nu_{R}}\right) d x \leq C e^{-R} R^{N} .
$$

Putting together (5.53) and (5.54) we get, choosing $C$ suffficiently large and for all $\varepsilon \leq \varepsilon_{0}(R)$,

$$
\begin{aligned}
\int_{B_{R}} \bar{m}_{\varepsilon} L_{\varepsilon}\left(-\frac{\bar{w}_{\varepsilon}}{\bar{m}_{\varepsilon}}\right) d x & \geq \int_{B_{R}} \bar{m} L_{\varepsilon}\left(-\frac{\bar{w}}{\bar{m}}\right) d x \\
& +\int_{B_{R}}\left(\bar{m}_{\varepsilon}-\bar{m}+2 \nu_{R}\right) L_{\varepsilon}\left(-\frac{\bar{w}_{\varepsilon}-\bar{w}+2 \nabla \nu_{R}}{\bar{m}_{\varepsilon}-\bar{m}+2 \nu_{R}}\right) d x-C R^{N} e^{-R}
\end{aligned}
$$

Therefore, summing up (5.55), (5.51), (5.44) and (5.45), we conclude for all $\varepsilon \leq \varepsilon_{0}(R)$,

$$
\mathcal{E}_{\varepsilon}\left(\bar{m}_{\varepsilon}, \bar{w}_{\varepsilon}\right) \geq \mathcal{E}_{\varepsilon}(\bar{m}, \bar{w})+\mathcal{E}_{\varepsilon}\left(\bar{m}_{\varepsilon}-\bar{m}+2 \nu_{R}, \bar{w}_{\varepsilon}-\bar{w}+2 \nabla \nu_{R}\right)+o_{\varepsilon}(1)-C R^{b+N} e^{-R} .
$$

Let now $c_{R}=\frac{M-a}{M-a+2 \int_{\mathbb{R}^{N}} \nu_{R} d x}$. We have that $c_{R} \rightarrow 1$ as $R \rightarrow+\infty$ and $c_{R}<1$. In particular, $\left(c_{R}\left(\bar{m}_{\varepsilon}-\bar{m}+2 \nu_{R}\right), c_{R}\left(\bar{w}_{\varepsilon}-\bar{w}+2 \nabla \nu_{R}\right)\right) \in \mathcal{K}_{M-a}$. By the same computation as in (5.31), we get

$$
\begin{aligned}
& \quad c_{R} \mathcal{E}_{\varepsilon}\left(\bar{m}_{\varepsilon}-\bar{m}+2 \nu_{R}, \bar{w}_{\varepsilon}-\bar{w}+2 \nabla \nu_{R}\right) \\
& =\mathcal{E}_{\varepsilon}\left(c_{R}\left(\bar{m}_{\varepsilon}-\bar{m}+2 \nu_{R}\right), c_{R}\left(\bar{w}_{\varepsilon}-\bar{w}+2 \nabla \nu_{R}\right)\right)+\int_{\mathbb{R}^{N}} c_{R} F_{\varepsilon}\left(\bar{m}_{\varepsilon}-\bar{m}+2 \nu_{R}\right)-F_{\varepsilon}\left(c_{R}\left(\bar{m}_{\varepsilon}-\bar{m}+2 \nu_{R}\right)\right) d x \\
& \quad \geq \mathcal{E}_{\varepsilon}\left(c_{R}\left(\bar{m}_{\varepsilon}-\bar{m}+2 \nu_{R}\right), c_{R}\left(\bar{w}_{\varepsilon}-\bar{w}+2 \nabla \nu_{R}\right)\right) \\
& \quad+c_{R} \frac{c_{R}^{\alpha}-1}{\alpha+1} C_{f} \int_{\mathbb{R}^{N}}\left(\bar{m}_{\varepsilon}-\bar{m}+2 \nu_{R}\right)^{\alpha+1} d x-2 K\left(M-a+2 \int_{\mathbb{R}^{N}} \nu_{R} d x\right) \varepsilon^{\frac{N \alpha \gamma^{\prime}}{\gamma^{\prime}-N \alpha}} \cdot \quad \text { (5.57) }
\end{aligned}
$$

Observe that by (5.15) there exists $C$ independent of $\varepsilon$ such that

$$
0 \leq \int_{\mathbb{R}^{N}}\left(\bar{m}_{\varepsilon}-\bar{m}+2 \nu_{R}\right)^{\alpha+1} d x \leq\left(\left\|\bar{m}_{\varepsilon}\right\|_{\alpha+1}+\|\bar{m}\|_{\alpha+1}+\left\|2 \nu_{R}\right\|_{\alpha+1}\right)^{\alpha+1} \leq C .
$$

Therefore, (5.57) reads (recalling that $c_{R}<1$ and enlarging the constants $C, K$ ),

$$
\begin{aligned}
c_{R} \mathcal{E}_{\varepsilon}\left(\bar{m}_{\varepsilon}-\bar{m}+2 \nu_{R}, \bar{w}_{\varepsilon}-\bar{w}+2 \nabla \nu_{R}\right) & \\
& \geq \mathcal{E}_{\varepsilon}\left(c_{R}\left(\bar{m}_{\varepsilon}-\bar{m}+2 \nu_{R}\right), c_{R}\left(\bar{w}_{\varepsilon}-\bar{w}+2 \nabla \nu_{R}\right)\right)+c_{R} \frac{c_{R}^{\alpha}-1}{\alpha+1} C-K M \varepsilon^{\frac{N \alpha \gamma^{\prime}}{\gamma^{\prime}-N \alpha}} \\
& \geq \tilde{e}_{\varepsilon}(M-a)+c_{R} \frac{c_{R}^{\alpha}-1}{\alpha+1} C-K M \varepsilon^{\frac{N \alpha \gamma^{\prime}}{\gamma^{\prime}-N \alpha}}
\end{aligned}
$$

Using this inequality, and using the fact that $\mathcal{E}_{\varepsilon}\left(\bar{m}_{\varepsilon}, \bar{w}_{\varepsilon}\right)=\tilde{e}_{\varepsilon}(M)$ and that $\mathcal{E}_{\varepsilon}(\bar{m}, \bar{w}) \geq \tilde{e}_{\varepsilon}(a)$, we obtain from (5.56)

$$
\begin{array}{r}
\tilde{e}_{\varepsilon}(M) \geq \tilde{e}_{\varepsilon}(a)+\tilde{e}_{\varepsilon}(M-a)+\left(1-c_{R}\right) \mathcal{E}_{\varepsilon}\left(\bar{m}_{\varepsilon}-\bar{m}+2 \nu_{R}, \bar{w}_{\varepsilon}-\bar{w}+2 \nabla \nu_{R}\right) \\
+C c_{R} \frac{c_{R}^{\alpha}-1}{\alpha+1}-K M \varepsilon^{\frac{N \alpha \gamma^{\prime}}{\gamma^{\prime}-N \alpha}}+o_{\varepsilon}(1)-C R^{b+N} e^{-R}
\end{array}
$$


Moreover by (5.29) we get that there exist $K=K(M-a)>0$ such that $\mathcal{E}_{\varepsilon}\left(\bar{m}_{\varepsilon}-\bar{m}+2 \nu_{R}, \bar{w}_{\varepsilon}-\right.$ $\left.\bar{w}+2 \nabla \nu_{R}\right) \geq-K$, therefore the previous inequality gives

$$
\tilde{e}_{\varepsilon}(M) \geq \tilde{e}_{\varepsilon}(a)+\tilde{e}_{\varepsilon}(M-a)-\left(1-c_{R}\right) K+C c_{R} \frac{c_{R}^{\alpha}-1}{\alpha+1}+o_{\varepsilon}(1)-C R^{b+N} e^{-R} .
$$

By Lemma [5.5, we get that

$$
\tilde{e}_{\varepsilon}(M) \leq \tilde{e}_{\varepsilon}(a)+\tilde{e}_{\varepsilon}(M-a)-C(a, M),
$$

where $C(a, M)>0$ for $a<M$ and $C(M, M)=0$. This implies in particular that

$$
0>-C(a, M) \geq-\left(1-c_{R}\right) K+C c_{R} \frac{c_{R}^{\alpha}-1}{\alpha+1}+o_{\varepsilon}(1)-C R^{b+N} e^{-R} .
$$

Recalling that $c_{R} \rightarrow 1$ as $R \rightarrow+\infty$, this gives a contradiction, choosing $R$ sufficiently large and $\varepsilon<\varepsilon_{0}(R)$.

An immediate corollary of the previous theorem is the following convergence result.

Corollary 5.7. Let $\left(\bar{u}_{\varepsilon}, \bar{m}_{\varepsilon}, \tilde{\lambda}_{\varepsilon}\right)$ and $(\bar{u}, \bar{m}, \bar{\lambda})$ be as in Proposition 5.3. Then,

$$
\bar{m}_{\varepsilon} \rightarrow \bar{m} \quad \text { in } L^{1}\left(\mathbb{R}^{N}\right) \text { and } L^{\alpha+1}\left(\mathbb{R}^{N}\right) \text {. }
$$

Finally for all $\eta>0$, there exist $R>0$ and $\varepsilon_{0}$ such that for all $\varepsilon \leq \varepsilon_{0}$,

$$
\int_{B(0, R)} \bar{m}_{\varepsilon} d x \geq M-\eta
$$

Proof. By Proposition 5.3 we get that $\bar{m}_{\varepsilon} \rightarrow \bar{m}$ almost everywhere, and by Theorem $\left[5.6 . \int_{\mathbb{R}^{N}} \bar{m}_{\varepsilon}=\right.$ $M=\int_{\mathbb{R}^{N}} \bar{m}$. This implies the convergence in $L^{1}\left(\mathbb{R}^{N}\right)$. Indeed, by Fatou lemma

$$
2 M \leq \liminf _{\varepsilon} \int_{\mathbb{R}^{N}} \bar{m}_{\varepsilon}+\bar{m}-\left|\bar{m}_{\varepsilon}-\bar{m}\right| d x \leq 2 M-\limsup _{\varepsilon} \int_{\mathbb{R}^{N}}\left|\bar{m}_{\varepsilon}-\bar{m}\right| d x .
$$

Moreover, recalling (5.16), we get that

$$
\left\|\bar{m}_{\varepsilon}-\bar{m}\right\|_{L^{\alpha+1}\left(\mathbb{R}^{N}\right)}^{\alpha+1} \leq\left\|\bar{m}_{\varepsilon}-\bar{m}\right\|_{L^{1}\left(\mathbb{R}^{N}\right)}\left(\|\bar{m}\|_{L^{\infty}\left(\mathbb{R}^{N}\right)}+\left\|\bar{m}_{\varepsilon}\right\|_{L^{\infty}\left(\mathbb{R}^{N}\right)}\right) \rightarrow 0 .
$$

Finally observe that for all $R$, by Remark 5.4 .

$$
\int_{B_{R}(0)} \bar{m}_{\varepsilon} d y \geq \int_{B_{R}(0)} \bar{m} d y-\int_{B_{R}(0)}\left|\bar{m}_{\varepsilon}-\bar{m}\right| d y \geq M-C R^{N-1} e^{-R}-\int_{\mathbb{R}^{N}}\left|\bar{m}_{\varepsilon}-\bar{m}\right| d y .
$$

So, using the $L^{1}$ convergence we conclude the desired estimate.

\subsection{Existence of ground states.}

In this subsection we aim at proving that as $\varepsilon$ goes to zero, $\left(\bar{u}_{\varepsilon}, \bar{m}_{\varepsilon}, \tilde{\lambda}_{\varepsilon}\right)$ converges to a solution of the limiting MFG system (1.14), without potential terms. In particular, we will prove Theorem 1.3 .

We first need a $\Gamma$-convergence type result, proved in the following lemma.

Lemma 5.8. Let $\left(m_{\varepsilon}, w_{\varepsilon}\right),(m, w) \in \mathcal{K}_{1, M}$ be such that $m_{\varepsilon} \rightarrow m$ in $L^{1} \cap L^{\alpha+1}\left(\mathbb{R}^{N}\right)$ and $w_{\varepsilon} \rightarrow w$ weakly in $L^{q}\left(\mathbb{R}^{N}\right)$ for some $q>1$. Then

$$
\lim \inf _{\varepsilon} \mathcal{E}_{\varepsilon}\left(m_{\varepsilon}, w_{\varepsilon}\right) \geq \mathcal{E}_{0}(m, w),
$$

where $\mathcal{E}_{0}$ is defined in (1.16).

Let $(m, w) \in \mathcal{K}_{1, M}$ be such that $m\left(1+|y|^{b}\right) \in L^{1}\left(\mathbb{R}^{N}\right)$. Then

$$
\lim _{\varepsilon} \mathcal{E}_{\varepsilon}\left(m\left(\cdot-y_{\varepsilon}\right), w\left(\cdot-y_{\varepsilon}\right)\right) \leq \mathcal{E}_{0}(m, w) .
$$


Proof. We recall that $L_{\varepsilon}(q) \rightarrow C_{L}|q|^{\gamma^{\prime}}$ uniformly in $\mathbb{R}^{N}$ by (5.11) and $F_{\varepsilon}(m) \rightarrow-\frac{1}{\alpha+1} m^{\alpha+1}$ uniformly in $[0,+\infty)$ by (5.7). Moreover we observe that the energy $\mathcal{E}_{0}$ is lower semicontinuous with respect to weak $L^{q}$ convergence of $w$ and strong $L^{\alpha+1} \cap L^{1}$ convergence of $m$. Since $V \geq 0$, we get that

$$
\begin{aligned}
\lim \inf _{\varepsilon} \mathcal{E}_{\varepsilon}\left(m_{\varepsilon}, w_{\varepsilon}\right) \geq \lim \inf _{\varepsilon} \int_{\mathbb{R}^{N}} m_{\varepsilon} L_{\varepsilon}\left(-\frac{w_{\varepsilon}}{m_{\varepsilon}}\right)+F_{\varepsilon}\left(m_{\varepsilon}\right) d x \\
\geq \liminf _{\varepsilon} \int_{\mathbb{R}^{N}} C_{L} m_{\varepsilon}^{1-\gamma^{\prime}}\left|w_{\varepsilon}\right|^{\gamma^{\prime}}-\frac{C_{f}}{\alpha+1} m_{\varepsilon}^{\alpha+1} d x \\
\quad \geq \int_{\mathbb{R}^{N}} C_{L} m^{1-\gamma^{\prime}}|w|^{\gamma^{\prime}}-\frac{C_{f}}{\alpha+1} m^{\alpha+1} d x=\mathcal{E}_{0}(m, w) .
\end{aligned}
$$

Now we observe that for all $m$ such that $m\left(1+|y|^{b}\right) \in L^{1}\left(\mathbb{R}^{N}\right)$, using (5.3), we get that

$$
\lim _{\varepsilon \rightarrow 0} \int_{\mathbb{R}^{N}} m\left(y+y_{\varepsilon}\right) V_{\varepsilon}\left(y+y_{\varepsilon}\right) d y \leq \lim _{\varepsilon} C_{V} \varepsilon^{\frac{N \alpha \gamma^{\prime}}{\gamma^{\prime}-\alpha N}} \int_{\mathbb{R}^{N}}(1+|y|)^{b} m(y) d y=0 .
$$

Therefore, recalling again the uniform convergence of $L_{\varepsilon}(q) \rightarrow C_{L}|q|^{\gamma^{\prime}}$ and $F_{\varepsilon}(m) \rightarrow-\frac{1}{\alpha+1} m^{\alpha+1}$, we conclude (noting that if we translate $m, w$ of $y_{\varepsilon}$ the energy $\mathcal{E}_{0}$ remains the same)

$$
\lim _{\varepsilon} \mathcal{E}_{\varepsilon}\left(m\left(\cdot-y_{\varepsilon}\right), w\left(\cdot-y_{\varepsilon}\right)\right)=\mathcal{E}_{0}(m, w)+\lim _{\varepsilon \rightarrow 0} \int_{\mathbb{R}^{N}} m\left(y+y_{\varepsilon}\right) V_{\varepsilon}\left(y+y_{\varepsilon}\right) d y \leq \mathcal{E}_{0}(m, w) .
$$

Proof of Theorem 1.3. We first show that $(\bar{u}, \bar{m})$ obtained in Proposition 5.3 are associated to minimizers of an appropriate energy, without potential term, so that (1.15) holds.

Note that $(\bar{m}, \bar{w}) \in \mathcal{K}_{1, M}$ where $\bar{w}=-\bar{m} \nabla H_{0}(\nabla \bar{u})$, due to Proposition 5.3 and Theorem 5.6 and $\bar{m}\left(1+|y|^{b}\right) \in L^{1}\left(\mathbb{R}^{N}\right)$ by the exponential decay (5.26). Moreover $\bar{m}_{\varepsilon} \rightarrow \bar{m}$ in $L^{1} \cap L^{\alpha+1}$ by Corollary 5.7 and $\bar{w}_{\varepsilon}=-\bar{m}_{\varepsilon} \nabla H_{\varepsilon}\left(\nabla \bar{u}_{\varepsilon}\right) \rightarrow \bar{w}=-\bar{m} \nabla H_{0}(\nabla \bar{u})$ locally uniformly (by Proposition 5.3) and weakly in $L^{\frac{\gamma^{\prime}(\alpha+1)}{\gamma^{\prime}+\alpha}}$ by the same argument as in the proof of Theorem 3.3 .

Let now $(m, w) \in \mathcal{K}_{1, M}$ be such that $m\left(1+|y|^{b}\right) \in L^{1}\left(\mathbb{R}^{N}\right)$. Using the minimality of $\left(\bar{m}_{\varepsilon}, \bar{w}_{\varepsilon}\right)$, (5.61) and (5.62), we conclude that

$$
\mathcal{E}_{0}(m, w) \geq \lim _{\varepsilon} \mathcal{E}_{\varepsilon}\left(m\left(\cdot-y_{\varepsilon}\right), w\left(\cdot-y_{\varepsilon}\right)\right) \geq \lim _{\varepsilon} \mathcal{E}_{\varepsilon}\left(\bar{m}_{\varepsilon}, \bar{w}_{\varepsilon}\right) \geq \mathcal{E}_{0}(\bar{m}, \bar{w}) .
$$

This implies (1.15).

To obtain the first part of the theorem, that is the existence of a solution to (1.14), we need to prove that the function $g$ appearing in Proposition 5.3 is actually zero on $\mathbb{R}^{N}$. To do that, we derive a better estimate on the term $V_{\varepsilon}\left(y+y_{\varepsilon}\right)$, in particular we show that $V_{\varepsilon}\left(y+y_{\varepsilon}\right) \rightarrow 0$ locally uniformly in $\mathbb{R}^{N}$.

By minimality of $\left(\bar{m}_{\varepsilon}, \bar{w}_{\varepsilon}\right)$ and $(\bar{m}, \bar{w})$, (5.11), (5.7) and (5.63) we get that

$$
\begin{aligned}
\mathcal{E}_{\varepsilon}\left(\bar{m}_{\varepsilon}, \bar{w}_{\varepsilon}\right) \leq & \mathcal{E}_{\varepsilon}\left(\bar{m}\left(\cdot+y_{\varepsilon}\right), \bar{w}\left(\cdot+y_{\varepsilon}\right)\right) \\
& \leq \mathcal{E}_{0}(\bar{m}, \bar{w})+\int_{\mathbb{R}^{N}} \bar{m}\left(y+y_{\varepsilon}\right) V_{\varepsilon}\left(y+y_{\varepsilon}\right) d y+C \varepsilon^{\frac{N \alpha \gamma^{\prime}}{\gamma^{\prime}-N \alpha}} \leq \mathcal{E}_{0}\left(\bar{m}_{\varepsilon}, \bar{w}_{\varepsilon}\right)+C_{1} \frac{N \alpha \gamma^{\prime}}{\gamma^{\prime}-N \alpha} .
\end{aligned}
$$

Again using (5.7) and (5.11) we get

$$
\mathcal{E}_{0}\left(\bar{m}_{\varepsilon}, \bar{w}_{\varepsilon}\right)+C_{1} \varepsilon^{\frac{N \alpha \gamma^{\prime}}{\gamma^{\prime}-N \alpha}} \leq \int_{\mathbb{R}^{N}} \bar{m}_{\varepsilon} L_{\varepsilon}\left(-\frac{\bar{w}_{\varepsilon}}{\bar{m}_{\varepsilon}}\right)+F_{\varepsilon}\left(\bar{m}_{\varepsilon}\right) d y+C \varepsilon^{\frac{N \alpha \gamma^{\prime}}{\gamma^{\prime}-\alpha N}} M+C \varepsilon^{\frac{N \alpha \gamma^{\prime}}{\gamma^{\prime}-N \alpha}}
$$

So, putting together the last two inequalities, we conclude that

$$
\int_{\mathbb{R}^{N}} \bar{m}_{\varepsilon} V_{\varepsilon}\left(y+y_{\varepsilon}\right) d y \leq C \varepsilon^{\frac{N \alpha \gamma^{\prime}}{\gamma^{\prime}-N \alpha}}
$$


Recalling (5.2), this implies that for all $R>0$, we get

$$
C_{V}^{-1}\left(\max \left\{\varepsilon^{\frac{\gamma^{\prime}}{\gamma^{\prime}-\alpha N}}\left|y_{\varepsilon}\right|-\varepsilon^{\frac{\gamma^{\prime}}{\gamma^{\prime}-\alpha N}} R-C_{V}, 0\right\}\right)^{b} \int_{B(0, R)} \bar{m}_{\varepsilon} d y \leq C
$$

Using (5.60), we conclude that there exists $C>0$ such that

$$
\varepsilon^{\frac{\gamma^{\prime}}{\gamma^{\prime}-\alpha N}}\left|y_{\varepsilon}\right| \leq C \text {. }
$$

In turns this gives, recalling again (5.2), that

$$
0 \leq V_{\varepsilon}\left(y+y_{\varepsilon}\right) \leq C_{V} \varepsilon^{\frac{N \alpha \gamma^{\prime}}{\gamma^{\prime}-\alpha N}}\left(1+\varepsilon^{\frac{\gamma^{\prime}}{\gamma^{\prime}-\alpha N}}|y|+\varepsilon^{\frac{\gamma^{\prime}}{\gamma^{\prime}-\alpha N}}\left|y_{\varepsilon}\right|\right)^{b} \leq C \varepsilon^{\frac{N \alpha \gamma^{\prime}}{\gamma^{\prime}-\alpha N}}(1+|y|)^{b}
$$

which implies that $V_{\varepsilon}\left(y+y_{\varepsilon}\right) \rightarrow 0$ locally uniformly.

Remark 5.9. If $H$ and $f$ satisfy the growth conditions (1.2) and (1.3), arguing as before one has that there exists a classical solution to the potential-free version of (1.1),

$$
\left\{\begin{array}{l}
-\Delta u+H(\nabla u)+\lambda=f(m) \\
-\Delta m-\operatorname{div}(\nabla H(\nabla u) m)=0 \\
\int_{\mathbb{R}^{N}} m=M .
\end{array}\right.
$$

In addition, $(m,-\nabla H(\nabla u) m)$ is a minimizer of

$$
(m, w) \mapsto \int_{\mathbb{R}^{N}} m L\left(-\frac{w}{m}\right)+F(m) d x
$$

among $(m, w) \in \mathcal{K}_{1, M}, m\left(1+|y|^{b}\right) \in L^{1}\left(\mathbb{R}^{N}\right)$. This can be done as follows: start with a sequence $\left(u_{\delta}, m_{\delta}, \lambda_{\delta}\right)$ solving

$$
\left\{\begin{array}{l}
-\Delta u_{\delta}+H\left(\nabla u_{\delta}\right)+\lambda_{\delta}=f\left(m_{\delta}\right)+\delta|x|^{b} \\
-\Delta m_{\delta}-\operatorname{div}\left(\nabla H\left(\nabla u_{\delta}\right) m_{\delta}\right)=0 \\
\int_{\mathbb{R}^{N}} m_{\delta}=M
\end{array}\right.
$$

with $\delta=\delta_{n} \rightarrow 0$. Such a sequence exists by Theorem 1.1. The problem of passing to the limit in (5.67) to obtain (5.66) is the same as passing to the limit in (5.13), and it is even simpler: in (5.13), one has to be careful as the Hamiltonian $H_{\varepsilon}$ and the coupling $f_{\varepsilon}$ vary as $\varepsilon \rightarrow 0$ (still, they converge uniformly), while in (5.67) they are fixed, and only the potential is vanishing. We observe that $b>0$ could be chosen arbitrarily, the perturbation $\delta|x|^{b}$ always disappears in the limit. Still, the limit $m, u$ somehow retains a memory of $b$ in terms of energy properties: $m$ minimizes an energy among competitors satisfying $m\left(1+|y|^{b}\right) \in L^{1}\left(\mathbb{R}^{N}\right)$.

Remark 5.10. We stress that uniqueness of solutions for (1.14) does not hold in general; for example, a triple $(u, m, \lambda)$ solving the system may be translated in space to obtain a full family of solutions. On the other hand, a more subtle issue is the uniqueness of $m$ in the second equation (with $\nabla u$ fixed), that is, if $\left(u, m_{1}, \lambda\right)$ and $\left(u, m_{2}, \lambda\right)$ are solutions, then $m_{1} \equiv m_{2}$. This property is intimately related to the ergodic behaviour of the optimal trajectory $d X_{s}=-\nabla H_{0}\left(\nabla u\left(X_{s}\right)\right) d s+$ $\sqrt{2 \varepsilon} d B_{s}$ (see, for example, [11] and references therein). It is well-known that uniqueness for the Kolmogorov equation is guaranteed by the existence of a so-called Lyapunov function; in our cases, it can be checked that $u$ itself (or increasing functions of $u$, as in (5.28)) acts as a Lyapunov function, so uniqueness of $m$ and ergodicity holds for (1.14) and (1.1).

\subsection{Concentration of mass}

The last problem we address is the localization of the point $y_{\varepsilon}$, to conclude the proof of Theorem 1.2. Rewriting (5.60) in view of (5.1) and (5.18), we get that for all $\eta>0$ there exist $R, \varepsilon_{0}$ such that for all $\varepsilon \leq \varepsilon_{0}$, 


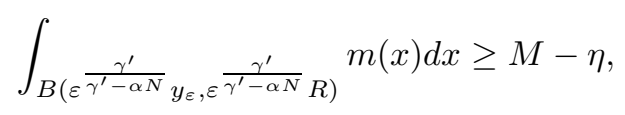

where $m$ is the classical solution to (1.1) given in Theorem 1.1, and $\bar{m}_{\varepsilon}(y)=\varepsilon^{\frac{N \gamma^{\prime}}{\gamma^{\prime}-\alpha N}} m\left(\varepsilon^{\frac{\gamma^{\prime}}{\gamma^{\prime}-\alpha N}} y+\right.$ $\left.\varepsilon^{\frac{\gamma^{\prime}}{\gamma^{\prime}-\alpha N}} y_{\varepsilon}\right)$.

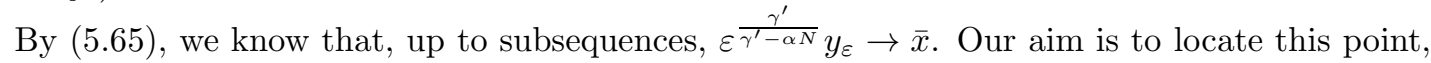
which is the point where mass concentrates. We need a preliminary lemma stating the existence of suitable competitors that will be used in the sequel.

Lemma 5.11. For all $\varepsilon \leq \varepsilon_{0}$, there exists $\left(\hat{m}_{\varepsilon}, \hat{w}_{\varepsilon}\right) \in \mathcal{K}_{1, M}$ that minimize

$$
(m, w) \mapsto \int_{\mathbb{R}^{N}} m L_{\varepsilon}\left(-\frac{w}{m}\right)+F_{\varepsilon}(m) d y
$$

among $(m, w) \in \mathcal{K}_{1, M}, m\left(1+|y|^{b}\right) \in L^{1}\left(\mathbb{R}^{N}\right)$. Moreover, for some positive constants $c_{1}, c_{2}$ independent of $\varepsilon$,

$$
\hat{m}_{\varepsilon}(y) \leq c_{1} e^{-c_{2}|y|} \quad \text { on } \mathbb{R}^{N} .
$$

Proof. The existence of $\left(\hat{m}_{\varepsilon}, \hat{w}_{\varepsilon}\right)$ is stated in Remark 5.9, together with a solution $\left(\hat{u}_{\varepsilon}, \hat{m}_{\varepsilon}, \hat{\lambda}_{\varepsilon}\right)$ to the associated MFG system as the optimality conditions (see (5.71) below). To obtain the uniform exponential decay, we can argue by Lyapunov functions as in Proposition 5.3 , here, we have to be careful, since the argument in Proposition 5.3 mainly require

$$
f_{\varepsilon}\left(\hat{m}_{\varepsilon}\right)-\hat{\lambda}_{\varepsilon}-H_{\varepsilon}(0) \geq-\hat{\lambda}_{\varepsilon} / 2>0
$$

outside some fixed ball $B_{r}(0)$. This claim can be proved as follows: first, $-\hat{\lambda}_{\varepsilon}$ is bounded away from zero for $\varepsilon$ small. Indeed,

$$
\hat{\lambda}_{\varepsilon} M=\int_{\mathbb{R}^{N}} \hat{m}_{\varepsilon} L_{\varepsilon}\left(-\frac{\hat{w}_{\varepsilon}}{\hat{m}_{\varepsilon}}\right)+f_{\varepsilon}\left(\hat{m}_{\varepsilon}\right) \hat{m}_{\varepsilon} d y \leq \mathcal{E}_{\varepsilon}\left(\bar{m}_{\varepsilon}, \bar{w}_{\varepsilon}\right)+o_{\varepsilon}(1) \leq-C .
$$

The inequality follows by minimality of $\left(\hat{m}_{\varepsilon}, \hat{w}_{\varepsilon}\right)$ and $\left(\bar{m}_{\varepsilon}, \bar{w}_{\varepsilon}\right)$, and (rescaled) (4.24).

We now prove that $\hat{m}_{\varepsilon}$ decays as $|x| \rightarrow \infty$ uniformly in $\varepsilon$. Note that $\hat{w}_{\varepsilon}=-\nabla H_{\varepsilon}\left(\nabla \hat{u}_{\varepsilon}\right) \hat{m}_{\varepsilon}$, where $\left(\hat{u}_{\varepsilon}, \hat{m}_{\varepsilon}, \hat{\lambda}_{\varepsilon}\right)$ solves

$$
\left\{\begin{array}{l}
-\Delta \hat{u}_{\varepsilon}+H_{\varepsilon}\left(\nabla \hat{u}_{\varepsilon}\right)+\lambda=f_{\varepsilon}\left(\hat{m}_{\varepsilon}\right) \\
-\Delta \hat{m}_{\varepsilon}-\operatorname{div}\left(\nabla H_{\varepsilon}\left(\nabla \hat{u}_{\varepsilon}\right) \hat{m}_{\varepsilon}\right)=0 \\
\int_{\mathbb{R}^{N}} \hat{m}_{\varepsilon}=M .
\end{array}\right.
$$

We derive local estimates for $\hat{u}_{\varepsilon}$ and $\hat{m}_{\varepsilon}$. We shift the $x$-variable so that $\hat{u}_{\varepsilon}(0)=0=\min _{\mathbb{R}^{N}} \hat{u}_{\varepsilon}$ for all $\varepsilon$. Choose $p>N$ such that

$$
\alpha<\frac{\gamma^{\prime}}{p}<\frac{\gamma^{\prime}}{N}
$$

If one considers the HJB equation solved by $\hat{u}_{\varepsilon}$, recalling (5.5) and (5.9), Theorem 2.5 gives the existence of $C>0$ such that

$$
\left\|\nabla \hat{u}_{\varepsilon}\right\|_{L^{\infty}\left(B_{2 R}\left(x_{0}\right)\right)} \leq K\left(\left\|\hat{m}_{\varepsilon}\right\|_{L^{\infty}\left(B_{4 R}\left(x_{0}\right)\right)}^{\alpha}+1\right)^{\frac{1}{\gamma}} .
$$

Note that $C>0$ does not depend on $\varepsilon$ and $x_{0}$. Turning to the Kolmogorov equation, again by (5.9) and Proposition 2.4.

$$
\left\|\hat{m}_{\varepsilon}\right\|_{W^{1, p}\left(B_{R}\left(x_{0}\right)\right)} \leq C\left(\left\|\nabla \hat{u}_{\varepsilon}\right\|_{L^{\infty}\left(B_{2 R}\left(x_{0}\right)\right)}^{\gamma-1}+1\right)\left\|m_{\varepsilon}\right\|_{L^{p}\left(B_{2 R}\left(x_{0}\right)\right)} .
$$

By the previous $L^{\infty}$ estimate on $\nabla u_{\varepsilon}$ and interpolation of the $L^{p}$ norm of $m$ between $L^{1}$ and $L^{\infty}$ we get

$$
\left\|\hat{m}_{\varepsilon}\right\|_{W^{1, p}\left(B_{R}\left(x_{0}\right)\right)} \leq C\left(\left\|\hat{m}_{\varepsilon}\right\|_{L^{\infty}\left(B_{4 R}\left(x_{0}\right)\right)}^{\frac{\alpha}{\gamma^{\prime}}}+1\right)\left\|\hat{m}_{\varepsilon}\right\|_{L^{1}\left(B_{4 R}\left(x_{0}\right)\right)}^{1 / p}\left\|\hat{m}_{\varepsilon}\right\|_{L^{\infty}\left(B_{4 R}\left(x_{0}\right)\right)}^{1-1 / p} .
$$


Recall that $\left\|\hat{m}_{\varepsilon}\right\|_{L^{1}\left(B_{4 R}\left(x_{0}\right)\right)} \leq M$; then, since $p>N$, by Sobolev embeddings we obtain that for some $\beta>0$,

$$
\left\|\hat{m}_{\varepsilon}\right\|_{C^{0, \beta}\left(B_{R}\left(x_{0}\right)\right)} \leq C\left(\left\|\hat{m}_{\varepsilon}\right\|_{L^{\infty}\left(\mathbb{R}^{N}\right)}^{\frac{\alpha}{\gamma^{\prime}}}+1\right)\left\|\hat{m}_{\varepsilon}\right\|_{L^{\infty}\left(\mathbb{R}^{N}\right)}^{1-1 / p} .
$$

First, since $C$ does not depend on $x_{0}$, this yields $\left\|\hat{m}_{\varepsilon}\right\|_{L^{\infty}\left(\mathbb{R}^{N}\right)} \leq C$, by the choice of $p<\gamma^{\prime} / \alpha$. Secondly, plugging back this estimate into (5.72), we conclude $\left\|\hat{m}_{\varepsilon}\right\|_{C^{0, \beta}\left(\mathbb{R}^{N}\right)} \leq C$.

Then, using these estimates, we get that up to subsequences, $\hat{\lambda}_{\varepsilon} \rightarrow \hat{\lambda}, \hat{u}_{\varepsilon} \rightarrow \hat{u}$ locally uniformly in $C^{1}$, and $\hat{m}_{\varepsilon} \rightarrow \hat{m}$ locally uniformly, where $(\hat{u}, \hat{m}, \hat{\lambda})$ is a solution to (5.25) with $g \equiv 0$. Arguing exactly as in Proposition 5.3. we get that $\tilde{u}, \tilde{m}$ satisfy the estimates (5.26) (eventually modifying the constants). Moreover $\int_{\mathbb{R}^{N}} \hat{m} d x=a \in(0, M]$. Observe now that Lemma [5.5 and Theorem 5.6 hold also for the energy (5.69), since it coincides with the energy $\mathcal{E}_{\varepsilon}$ without the potential term $\int_{\mathbb{R}^{N}} V_{\varepsilon} m d x$. Therefore we can apply Theorem 5.6 to $\hat{m}$, to conclude that actually $\int_{\mathbb{R}^{N}} \hat{m} d x=M$. So, by Corollary 5.7 we obtain that for all $\eta>0$, there exist $R>0$ and $\varepsilon_{0}$ such that for all $\varepsilon \leq \varepsilon_{0}$,

$$
\int_{B(0, R)} \hat{m}_{\varepsilon} d x \geq M-\eta
$$

By (5.72) and (5.73), using Lemma 2.2, we get that

$$
f_{\varepsilon}\left(\hat{m}_{\varepsilon}\right) \geq \frac{\hat{\lambda}_{\varepsilon}}{4}
$$

outside a ball $B_{r}(0)$. Since $H_{\varepsilon}(0) \rightarrow 0$, the claim

$$
f_{\varepsilon}\left(\hat{m}_{\varepsilon}\right)-\hat{\lambda}_{\varepsilon}-H_{\varepsilon}(0) \geq-\hat{\lambda}_{\varepsilon} / 2>0
$$

outside a ball $B_{r}(0)$ follows. As previously mentioned, me may now proceed and conclude as in Proposition 5.3. basically, (5.74) implies that $x \mapsto e^{k \hat{u}_{\varepsilon}(x)}$ acts as a Lyapunov function for $\hat{m}_{\varepsilon}$ for some small $k>0$, giving

$$
c \int_{\mathbb{R}^{N}} e^{k|x|-k_{1}} \hat{m}_{\varepsilon} \leq \int_{\mathbb{R}^{N}} e^{k \hat{u}_{\varepsilon}} \hat{m}_{\varepsilon} \leq C
$$

for all $\varepsilon$ small, that easily implies the pointwise exponential decay (5.70) of $\hat{m}_{\varepsilon}$ by Hölder regularity of $\hat{m}_{\varepsilon}$ itself.

For general potentials, the point where mass concentrates is a minimum for $V$.

Proposition 5.12. Up to subsequences, $\varepsilon^{\frac{\gamma^{\prime}}{\gamma^{\prime}-\alpha N}} y_{\varepsilon} \rightarrow \bar{x}$, where $V(\bar{x})=0$, i.e. $\bar{x}$ is a minimum of $V$.

Proof. Fix a generic $z \in \mathbb{R}^{N}$ and observe that $\left(\hat{m}_{\varepsilon}(\cdot+z), \hat{w}_{\varepsilon}(\cdot+z)\right)$ is still a minimizer of $\int m L_{\varepsilon}\left(-\frac{w}{m}\right)+F_{\varepsilon}(m)$. By minimality of $\left(\bar{m}_{\varepsilon}, \bar{w}_{\varepsilon}\right)$ and of $\left(\hat{m}_{\varepsilon}(\cdot+z), \hat{w}_{\varepsilon}(\cdot+z)\right)$, we get that

$$
\begin{aligned}
& \int_{\mathbb{R}^{N}} \bar{m}_{\varepsilon} L_{\varepsilon}\left(-\frac{\bar{w}_{\varepsilon}}{\bar{m}_{\varepsilon}}\right)+F_{\varepsilon}\left(\bar{m}_{\varepsilon}\right) d y+\int_{\mathbb{R}^{N}} \bar{m}_{\varepsilon}(y) V_{\varepsilon}\left(y+y_{\varepsilon}\right) d y=\mathcal{E}_{\varepsilon}\left(\bar{m}_{\varepsilon}, \bar{w}_{\varepsilon}\right) \\
& \quad \leq \mathcal{E}_{\varepsilon}\left(\hat{m}_{\varepsilon}(\cdot+z), \hat{w}_{\varepsilon}(\cdot+z)\right) \leq \int_{\mathbb{R}^{N}} \bar{m}_{\varepsilon} L_{\varepsilon}\left(-\frac{\bar{w}_{\varepsilon}}{\bar{m}_{\varepsilon}}\right)+F_{\varepsilon}\left(\bar{m}_{\varepsilon}\right)+\int_{\mathbb{R}^{N}} \hat{m}_{\varepsilon}(y+z) V_{\varepsilon}\left(y+y_{\varepsilon}\right) d y .
\end{aligned}
$$

In particular this gives that

$$
\int_{\mathbb{R}^{N}} \bar{m}_{\varepsilon}(y) V_{\varepsilon}\left(y+y_{\varepsilon}\right) d y \leq \int_{\mathbb{R}^{N}} \hat{m}_{\varepsilon}(y+z) V_{\varepsilon}\left(y+y_{\varepsilon}\right) d y=\int_{\mathbb{R}^{N}} \hat{m}_{\varepsilon}(y) V_{\varepsilon}\left(y+y_{\varepsilon}-z\right) d y \quad \forall z \in \mathbb{R}^{N} .
$$

Recalling the rescaling of $V_{\varepsilon}$ and of $\bar{m}_{\varepsilon}$ in (5.1), this is equivalent to

$$
\int_{\mathbb{R}^{N}} m(x) V(x) d x \leq \int_{\mathbb{R}^{N}} \hat{m}_{\varepsilon}(y) V\left(\varepsilon \frac{\gamma^{\prime}}{\gamma^{\prime}-\alpha N} y+\varepsilon^{\frac{\gamma^{\prime}}{\gamma^{\prime}-\alpha N}} y_{\varepsilon}-\varepsilon^{\frac{\gamma^{\prime}}{\gamma^{\prime}-\alpha N}} z\right) d y \quad \forall z \in \mathbb{R}^{N}
$$


where $m$ is the classical solution to (1.1) given in Theorem 1.1, such that $\bar{m}_{\varepsilon}(y)=\varepsilon^{\frac{N \gamma^{\prime}}{\gamma^{\prime}-\alpha N}} m\left(\frac{\varepsilon^{\gamma^{\prime}-\alpha N}}{\gamma^{\prime}} y+\right.$ $\left.\varepsilon^{\frac{\gamma^{\prime}}{\gamma^{\prime}-\alpha N}} y_{\varepsilon}\right)$.

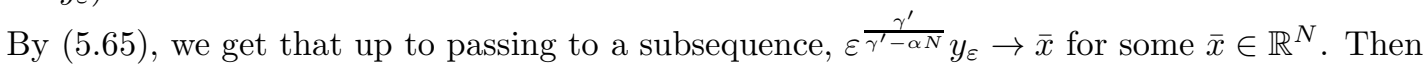
by (5.68), we get that

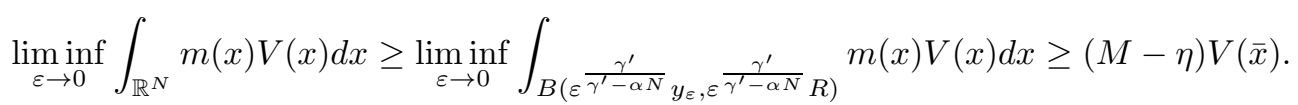

We fix $\bar{z}$ such that $V(\bar{z})=0$ and we choose in (5.76) $z=y_{\varepsilon}-\varepsilon^{-\frac{\gamma^{\prime}}{\gamma^{\prime}-\alpha N}} \bar{z}$. We have, by the Lebesgue convergence theorem and (5.70),

$$
\limsup _{\varepsilon \rightarrow 0} \int_{\mathbb{R}^{N}} \hat{m}_{\varepsilon}(y) V\left(\varepsilon^{\frac{\gamma^{\prime}}{\gamma^{\prime}-\alpha N}} y+\bar{z}\right) d y \leq \limsup _{\varepsilon \rightarrow 0} c_{1} \int_{\mathbb{R}^{N}} e^{-c_{2}|y|} V\left(\varepsilon^{\frac{\gamma^{\prime}}{\gamma^{\prime}-\alpha N}} y+\bar{z}\right) d y=0 .
$$

By (5.77), (5.78) and (5.76), we conclude $V(\bar{x})=0$.

If we assume that the potential $V$ has a finite number of minima and polynomial behavior, that is, it satisfies assumption (1.13), then we get that at the limit $\frac{\gamma^{\gamma^{\prime}}}{\gamma^{\prime}-\alpha N} y_{\varepsilon}$ selects at the limit the more stable minima of $V$, as we will show in the next proposition.

Proposition 5.13. Assume that $V$ satisfies assumption (1.13). Then, up to subsequences, there holds that

$$
\varepsilon^{\frac{\gamma^{\prime}}{\gamma^{-\alpha N}}} y_{\varepsilon} \rightarrow x_{i} \quad \text { as } \varepsilon \rightarrow 0
$$

where $i \in\left\{j=1, \ldots, n, \mid b_{j}=\max _{k} b_{k}\right\}$.

Proof. By Proposition 5.12 we know that up to subsequences, $\varepsilon^{\frac{\gamma^{\prime}}{\gamma^{\prime}-\alpha N}} y_{\varepsilon} \rightarrow x_{\iota}$ for some $\iota=1, \ldots n$. It remains to prove that $b_{\iota}=\max _{i} b_{i}$. Assume by contradiction that it is not true, and then $b_{\iota}<\max _{i} b_{i}$.

We compute for $j \in 1, \ldots n$, recalling the uniform exponential decay of $\hat{m}_{\varepsilon}$ given in (5.70),

$$
\begin{aligned}
& \int_{\mathbb{R}^{n}} \hat{m}_{\varepsilon}\left(y+y_{\varepsilon}-\right.\left.\varepsilon^{-\frac{\gamma^{\prime}}{\gamma^{\prime}-\alpha N}} x_{j}\right) V_{\varepsilon}\left(y+y_{\varepsilon}\right) d y=\int_{\mathbb{R}^{n}} \hat{m}_{\varepsilon}(y) V_{\varepsilon}\left(y+\varepsilon^{-\frac{\gamma^{\prime}}{\gamma^{\prime}-\alpha N}} x_{j}\right) d y \\
& \leq C_{V} \varepsilon^{\frac{\gamma^{\prime} N \alpha}{\gamma^{\prime}-N \alpha}} \int_{\mathbb{R}^{n}} \hat{m}_{\varepsilon}(y) \varepsilon^{\frac{b_{j} \gamma^{\prime}}{\gamma^{\prime}-N \alpha}}|y|^{b_{j}} \prod_{i \neq j}\left|\varepsilon^{\frac{\gamma^{\prime}}{\gamma^{\prime}-N \alpha}} y-x_{i}+x_{j}\right|^{b_{i}} d y \\
& \leq C \varepsilon^{\frac{\gamma^{\prime}\left(N \alpha+b_{j}\right)}{\gamma^{\prime}-N \alpha}} \int_{\mathbb{R}^{n}} \hat{m}_{\varepsilon}(y)|y|^{b_{j}} \prod_{i \neq j}\left|y-x_{i}+x_{j}\right|^{b_{i}} d y \leq C \varepsilon^{\frac{\gamma^{\prime}\left(N \alpha+b_{j}\right)}{\gamma^{\prime}-N \alpha}}
\end{aligned}
$$

Note in particular that we can choose in the previous inequality $b_{j}=\max _{i} b_{i}$.

We get from (5.75) applied to $z=y_{\varepsilon}-\varepsilon^{-\frac{\gamma^{\prime}}{\gamma^{\prime}-\alpha N}} x_{j}$, where $j$ is such that $b_{j}=\max _{i} b_{i}$, and from (5.79) the following improvement of (5.64)

$$
\int_{B(0, R)} \bar{m}_{\varepsilon} V_{\varepsilon}\left(y+y_{\varepsilon}\right) d y \leq \int_{\mathbb{R}^{N}} \hat{m}_{\varepsilon}\left(y+y_{\varepsilon}-\varepsilon^{-\frac{\gamma^{\prime}}{\gamma^{\prime}-\alpha N}} x_{j}\right) V_{\varepsilon}\left(y+y_{\varepsilon}\right) d y \leq C \varepsilon^{\frac{\left(N \alpha+\max b_{i}\right) \gamma^{\prime}}{\gamma^{\prime}-N \alpha}}
$$

for all $R \geq 0$. We choose $R>0$ sufficiently large such that $\int_{B(0, R)} \bar{m}_{\varepsilon} d y \geq \frac{M}{2}$. Recalling the rescaling of $V$, (5.80) implies that

$$
C \varepsilon^{\frac{\max b_{j} \gamma^{\prime}}{\gamma^{\prime}-N \alpha}} \geq \frac{M}{2} C_{V}^{-1} \min _{y \in B(0, R)} \prod_{j=1}^{n}\left|\varepsilon^{\frac{\gamma^{\prime}}{\gamma^{\prime}-N \alpha}} y+\varepsilon^{\frac{\gamma^{\prime}}{\gamma^{\prime}-N \alpha}} y_{\varepsilon}-x_{j}\right|^{b_{j}}
$$


Note that for $\varepsilon$ sufficiently small $\left|\varepsilon \frac{\gamma^{\prime}}{\gamma^{\prime}-N \alpha} y+\varepsilon \frac{\gamma^{\prime}}{\gamma^{\prime}-N \alpha} y_{\varepsilon}-x_{j}\right| \geq \delta>0$ for all $i \neq \iota$ and all $y \in B(0, R)$. So, by (5.81) we get that there exists $C>0$ for which

$$
\min _{y \in B(0, R)}\left|\varepsilon^{\frac{\gamma^{\prime}}{\gamma^{\prime}-N \alpha}} y+\varepsilon^{\frac{\gamma^{\prime}}{\gamma^{\prime}-N \alpha}} y_{\varepsilon}-x_{\iota}\right|^{b_{\iota}} \leq C \varepsilon^{\frac{\max b_{j} \gamma^{\prime}}{\gamma^{\prime}-N \alpha}}
$$

and then

$$
\left|\hat{y}_{\varepsilon}-\varepsilon^{-\frac{\gamma^{\prime}}{\gamma^{\prime}-N \alpha}} x_{\iota}\right|^{b_{\iota}}=\min _{y \in B(0, R)}\left|y+y_{\varepsilon}-\varepsilon^{-\frac{\gamma^{\prime}}{\gamma^{\prime}-N \alpha}} x_{\iota}\right|^{b_{\iota}} \leq C \varepsilon^{\frac{\left(\max b_{j}-b_{\iota}\right) \gamma^{\prime}}{\gamma^{\prime}-N \alpha}} \rightarrow 0
$$

for some $\hat{y}_{\varepsilon} \in B\left(y_{\varepsilon}, R\right)$. Let $z_{\varepsilon}=\hat{y}_{\varepsilon}-y_{\varepsilon} \in B(0, R)$. Up to subsequences we can assume that $z_{\varepsilon} \rightarrow \bar{z} \in B(0, R)$.

We use now (5.80), recalling assumption (1.13), we get that

$$
\begin{aligned}
C \varepsilon^{\frac{\max b_{j} \gamma^{\prime}}{\gamma^{\prime}-N \alpha}} \geq C_{V}^{-1} \int_{B(0, R)} \bar{m}_{\varepsilon}(y) \prod_{j=1}^{n}\left|\varepsilon^{\frac{\gamma^{\prime}}{\gamma^{\prime}-N \alpha}} y+\varepsilon^{\frac{\gamma^{\prime}}{\gamma^{\prime}-N \alpha}} y_{\varepsilon}-x_{j}\right|^{b_{j}} d y \\
\quad \geq c_{1} \varepsilon^{\frac{b_{\iota} \gamma^{\prime}}{\gamma^{\prime}-N \alpha}} \int_{B(0, R)} \bar{m}_{\varepsilon}(y)\left|y-z_{\varepsilon}+\hat{y}_{\varepsilon}-\varepsilon^{-\frac{\gamma^{\prime}}{\gamma^{\prime}-N \alpha}} x_{\iota}\right|^{b_{\iota}} d y .
\end{aligned}
$$

In particular this implies that

$$
\lim _{\varepsilon \rightarrow 0} \int_{B(0, R)} \bar{m}_{\varepsilon}(y)\left|y-z_{\varepsilon}+\hat{y}_{\varepsilon}-\varepsilon^{-\frac{\gamma^{\prime}}{\gamma^{\prime}-N \alpha}} x_{\iota}\right|^{b_{\iota}} d y=0 .
$$

Recalling that $\bar{m}_{\varepsilon} \rightarrow \bar{m}$ locally uniformly (see (5.24) ), that $\hat{y}_{\varepsilon}-\varepsilon^{-\frac{\gamma^{\prime}}{\gamma^{\prime}-N \alpha}} x_{\iota} \rightarrow 0$ by (5.82), and that $z_{\varepsilon} \rightarrow \bar{z}$, we get

$$
\lim _{\varepsilon \rightarrow 0} \int_{B(0, R)} \bar{m}_{\varepsilon}(y)\left|y-z_{\varepsilon}+\hat{y}_{\varepsilon}-\varepsilon^{-\frac{\gamma^{\prime}}{\gamma^{\prime}-N \alpha}} x_{\iota}\right|^{b_{\iota}} d y=\int_{B(0, R)} \bar{m}(y)|y-\bar{z}|^{b_{\iota}} d y>0 .
$$

This gives a contradiction with (5.83).

As a consequence of the previous results, we can conclude with the

Proof of Theorem 1.2. Setting $x_{\varepsilon}=\varepsilon^{\frac{\gamma^{\prime}}{\gamma^{\prime}-\alpha N}} y_{\varepsilon}$, it suffices to recall (5.68) and Propositions 5.12, 5.13 .

\section{References}

[1] S. Agmon. The $L_{p}$ approach to the dirichlet problem. part I: regularity theorems. Ann. Sc. Norm. Super. Pisa Cl. Sci. (5), 13(4):405-448, 1959.

[2] A. Arapostathis, A. Biswas, and J. Carroll. On solutions of mean field games with ergodic cost. Journal de Mathématiques Pures et Appliquées, 107(2):205 - 251, 2017.

[3] M. Bardi and F. S. Priuli. Linear-quadratic $N$-person and mean-field games with ergodic cost. SIAM J. Control Optim., 52(5):3022-3052, 2014.

[4] G. Barles and J. Meireles. On unbounded solutions of ergodic problems in $\mathbb{R}^{m}$ for viscous HamiltonJacobi equations. Comm. Partial Differential Equations, 41(12):1985-2003, 2016.

[5] G. Barles, A. Porretta, and T. T. Tchamba. On the large time behavior of solutions of the Dirichlet problem for subquadratic viscous Hamilton-Jacobi equations. J. Math. Pures Appl. (9), 94(5):497519,2010 .

[6] J. M. Borwein and J. D. Vanderwerff. Convex functions: constructions, characterizations and counterexamples, volume 109 of Encyclopedia of Mathematics and its Applications. Cambridge University Press, Cambridge, 2010. 
[7] H. Brézis and E. Lieb. A relation between pointwise convergence of functions and convergence of functionals. Proc. Amer. Math. Soc., 88(3):486-490, 1983.

[8] A. Briani and P. Cardaliaguet. Stable solutions in potential mean field game systems. NoDEA Nonlinear Differential Equations Appl., 25(1):25:1, 2018.

[9] P. Cardaliaguet and P. J. Graber. Mean field games systems of first order. ESAIM Control Optim. Calc. Var., 21(3):690-722, 2015.

[10] A. Cesaroni and M. Cirant. Introduction to variational methods for viscous ergodic mean-field games with local coupling. 2017. Lecture notes in Springer INdAM Series, to appear.

[11] M. Cirant. On the solvability of some ergodic control problems in $\mathbb{R}^{d}$. SIAM J. Control Optim., 52(6):4001-4026, 2014.

[12] M. Cirant. Stationary focusing mean-field games. Comm. Partial Differential Equations, 41(8):13241346, 2016.

[13] M. Cirant. On the existence of oscillating solutions in non-monotone mean-field games. arXiv preprint, https://arxiv.org/abs/1711.08047, 2017.

[14] M. Cirant and D. Tonon. Time-dependent focusing mean-field games: the sub-critical case. arXiv preprint, https://arxiv.org/abs/1704.04014, 2017.

[15] D. A. Gomes, L. Nurbekyan, and M. Prazeres. One-dimensional stationary mean-field games with local coupling. arXiv preprint, https://arxiv.org/abs/1611.08161, 2016.

[16] D. A. Gomes and E. Pimentel. Local regularity for mean-field games in the whole space. Minimax Theory Appl., 1(1):65-82, 2016.

[17] D. A. Gomes, E. A. Pimentel, and V. Voskanyan. Regularity theory for mean-field game systems. SpringerBriefs in Mathematics. Springer, 2016.

[18] M. Huang, R. P. Malhamé, and P. E. Caines. Large population stochastic dynamic games: closedloop McKean-Vlasov systems and the Nash certainty equivalence principle. Commun. Inf. Syst., 6(3):221-251, 2006.

[19] N. Ichihara. Recurrence and transience of optimal feedback processes associated with Bellman equations of ergodic type. SIAM Journal on Control and Optimization, 49(5):1938-1960, 2011.

[20] N. Ichihara. The generalized principal eigenvalue for Hamilton-Jacobi-Bellman equations of ergodic type. Ann. Inst. H. Poincaré Anal. Non Linéaire, 32(3):623-650, 2015.

[21] J.-M. Lasry and P.-L. Lions. Nonlinear elliptic equations with singular boundary conditions and stochastic control with state constraints. Math. Ann., 283(4):583-630, 1989.

[22] J.-M. Lasry and P.-L. Lions. Jeux à champ moyen. I. Le cas stationnaire. C. R. Math. Acad. Sci. Paris, 343(9):619-625, 2006.

[23] J.-M. Lasry and P.-L. Lions. Jeux à champ moyen. II. Horizon fini et contrôle optimal. C. R. Math. Acad. Sci. Paris, 343(10):679-684, 2006.

[24] J.-M. Lasry and P.-L. Lions. Mean field games. Jpn. J. Math., 2(1):229-260, 2007.

[25] P.-L. Lions. In cours au collége de france. www.college-de-france.fr.

[26] P.-L. Lions. The concentration-compactness principle in the calculus of variations. The locally compact case. I. Ann. Inst. H. Poincaré Anal. Non Linéaire, 1(2):109-145, 1984.

[27] A. R. Meszaros and F. J. Silva. On the variational formulation of some stationary second order mean field games systems. arXiv preprint, https://arxiv.org/abs/1704.02125, 2017.

[28] G. Metafune, D. Pallara, and A. Rhandi. Global properties of invariant measures. J. Funct. Anal., 223(2):396-424, 2005.

[29] A. Porretta. On the weak theory for mean field games systems. Boll. Unione Mat. Ital., pages 1-29, 2016. 
cirant@math. unipd.it Dipartimento di Matematica "Tullio Levi-Civita" Università di Padova via Trieste 63, 35121 Padova (Italy) 